\title{
ON WEAK SOLUTION OF SDE DRIVEN BY INHOMOGENEOUS SINGULAR LÉVY NOISE
}

\author{
TADEUSZ KULCZYCKI, ALEXEI KULIK, AND MICHAŁ RYZNAR
}

\begin{abstract}
We study a time-inhomogeneous SDE in $\mathbb{R}^{d}$ driven by a cylindrical Lévy process with independent coordinates which may have different scaling properties. Such a structure of the driving noise makes it strongly spatially inhomogeneous and complicates the analysis of the model significantly. We prove that the weak solution to the SDE is uniquely defined, is Markov, and has the strong Feller property. The heat kernel of the process is presented as a combination of an explicit 'principal part' and a 'residual part', subject to certain $L^{\infty}(d x) \otimes L^{1}(d y)$ and $L^{\infty}(d x) \otimes L^{\infty}(d y)$-estimates showing that this part is negligible in a short time, in a sense. The main tool of the construction is the analytic parametrix method, specially adapted to Lévy-type generators with strong spatial inhomogeneities.
\end{abstract}

\section{INTRODUCTION}

In this paper we study an SDE of the form

$$
d X_{t}=\int V_{t}\left(X_{t-}, z\right) N(d t, d z), \quad X_{0}=x \in \mathbb{R}^{d}, \quad t \geq 0,
$$

where $N(d t, d z)$ is a Poisson random measure, which corresponds to a symmetric Lévy process $Z=\left(Z_{t}, t \geq 0\right)$ in the usual sense that

$$
d Z_{t}=\int z N(d t, d z)
$$

Heuristically, the dynamics of the process $X$ can be described as follows: whenever the driving process has a jump with the altitude $\triangle_{t} Z=z$, the process $X$ makes the jump with the altitude $\triangle_{t} X=V_{t}\left(X_{t-}, z\right)$. Such a description can be made rigorous either if the total intensity of jumps for $Z$ is finite (and then the jumps can be processed one by one), or the jump coefficient $V_{t}(x, z)$ satisfies a proper version of the Lipschitz condition w.r.t. $x$ (and then the solution to (11) can be obtained by the Itô-Lévy stochastic calculus tools, e.g. [19, Section IV.9]). In both these cases, $X$ is a strong solution to (1), i.e. a process adapted to the natural filtration generated by the Lévy noise. In the current paper, we deal with a more sophisticated setting where the coefficient is assumed to be Hölder continuous, only. In this case, one can still expect to have $X$ uniquely defined in law as a weak solution to (11). The guideline here is provided by the classic diffusion theory [34, based on an analytic study of the backward Kolmogorov equation for the (formal) generator, associated with the SDE. Extension of this analytic theory to Lévy driven SDEs has been a subject of intensive studies, see the literature overview in Section 2.4 below. Such an extension is far from being straightforward; namely, because of high diversity of the possible structure of the Lévy noise, numerous new effects appear, often requiring specific methods to be treated. In the current paper we approach a quite challenging case, where the driving process $Z$ has the form

$$
Z=\left(Z^{1}, \ldots Z^{d}\right),
$$

T. Kulczycki and M. Ryznar were supported in part by the National Science Centre, Poland, grant no. 2019/35/B/ST1/01633.

A. Kulik has been supported through the DFG-NCN Beethoven Classic 3 programme, contract no. 2018/31/G/ST1/02252 (National Science Center, Poland) and SCHI-419/11-1 (DFG, Germany). 
with $Z^{i}, i=1, \ldots, d$ being independent scalar Lévy processes which have the weak scaling property (WSP), see (6) below. The jump coefficient will be assumed to have a natural form

$$
V_{t}(x, z)=A_{t}(x) z+U_{t}(x, z)
$$

with the linear part $A_{t}(x) z$ being principal, in a sense, for small $|z|$. Clearly, when $U \equiv 0$ equation (11) is equivalent to

$$
d X_{t}=A_{t}\left(X_{t-}\right) d Z_{t}, \quad X_{0}=x \in \mathbb{R}^{d} .
$$

We stress that even the case of $A_{t}(x)=A(x), U_{t}(x, z) \equiv 0$ and all $Z^{i}, i=1, \ldots, d$ having the same $\alpha$-stable distribution is quite complicated; for instance, corresponding transition probability densities may fail to be locally bounded. Such an effect appears if the distributions of a jump for various starting points are mutually singular; for a detailed discussion we refer to [22, Section 4], where such models are called essentially singular. The essential singularity in the above setting is caused by a combination of two features: the fact that the Lévy measure of the process (2) is supported by the collection of the coordinate axes in $\mathbb{R}^{d}$ and thus is singular w.r.t. the Lebesgue measure, and a non-trivial rotation provided by the matrix $A(x)$. In this paper we will make one more substantial step further and allow the one-dimensional components of the noise to have different laws. To outline the new difficulties which appear in this setting, let us consider for a while $Z$ with $\alpha_{i}$-stable components, $i=1, \ldots, d$. For small $t$, the law of $Z_{t}$ is mainly concentrated around the axis with the number $j=\operatorname{argmin}_{i} \alpha_{i}$, which combined with a non-trivial rotation makes the model quite difficult to analyze analytically.

The first steps in the study of essentially singular models have been made in [30], [28, [22] and [3]. In [30], the components of the noise were the same and $\alpha$-stable. The results of [30] were significantly extended in 3], where time-inhomogeneous model with a drift was studied. In 22] general stable-like models have been treated, where the stability index and the spherical kernel (i.e. the distribution of the jump direction) are $x$-dependent. In [28], instead of stable noise, a more general class of noises has been treated, satisfying weak scaling condition; see definition in Section 2 below. In this paper we extend these previous results in several directions. First, in the setting of [28], where the cylindrical noise has the same laws of the coordinates, we remove several hidden limitations. Namely,

- instead of the linear-in- $z$ coefficient $V(x, z)=A(x) z$, we consider the coefficients of the form (3) with a principal linear part and residual non-linearity;

- time-inhomogeneous models are engaged into study;

- instead of the Lipschitz continuity of the matrix coefficient $A(x)$, the Hölder continuity is assumed.

Second, we make a further substantial step, treating a cylindrical noise which has different laws of the coordinates. As we have explained before, such an extension leads to substantial analytical difficulties; in addition, quite new effects may appear because of different scaling for various coordinates. Namely, we will see in Example 2.7 that, in this setting, non-trivial assumptions on the Hölder indices of the coefficients should be made, in the striking contrast to the case of same coordinates, or the stable-like case studied in [22].

To provide a comprehensive analysis of the new effects which appear due to strongly inhomogeneous and singular Lévy noise, we restrict ourselves to models which do not contain a drift term; i.e. without a gradient term in the generator. Adding a drift term can lead to further complications because of possible lack of domination property in the case of the lower scaling index $\alpha<1$. It is visible that these problems can be resolved by the 'flow corrector' method introduced in [21], [32, see also a discussion in [22, Sections 6.1,6.2]; such an extension is a topic of our ongoing research.

We will prove existence and uniqueness of the weak solution to (1), which will be shown to be a time-inhomogeneous Markov process. We will also provide a representation of the 
transition probability density of this process as a sum of explicitly given 'principal part', and a 'residual part' subject to a set of estimates showing that this part is negligible in a short time, in a sense. The 'principal part' will be given in the form

$$
\widetilde{p}_{t, s}(x, y)=\frac{1}{\left|\operatorname{det} A_{t}(x)\right|} \widetilde{G}_{s-t}\left((y-x)\left(A_{t}(x)^{-1}\right)^{T}\right), \quad 0 \leq t<s, \quad x, y \in \mathbb{R}^{d},
$$

where $\widetilde{G}_{t}(\cdot)$ is the distribution density of $Z_{t}$. Clearly, as a function of $y$, this is the distribution density of the variable

$$
\tilde{X}_{s}^{t, x}=x+A_{t}(x)\left(Z_{s}-Z_{t}\right),
$$

which can be seen as a natural approximation to the value at the time instant $s$ of the solution to (1), which starts from the point $x$ at the time instant $t$.

It is worth mentioning that recently the existence of densities for SDEs driven by singular Lévy processes have been studied in [12] (cf. also [8]).

The structure of the rest of the paper is the following. In Section 2 we introduce the assumptions, formulate the main results, and provide a comprehensive discussion for them, based on examples and an overview of related results, available in the literature. Sections 3 - 5 contain the proofs. The proofs are rather technical, hence in order to improve readability we first explain the keystones of the proofs in Section 3 . Numerous estimates required in the main proof are deduced in Sections $4-5$.

\section{MAin RESUlts}

2.1. Assumptions. In this section, we collect all the assumptions we impose on our model. Let us begin with the description of scalar Lévy processes involved, as the coordinates, into the representation (2). Let the characteristic exponent $\psi$ of a one-dimensional, symmetric Lévy process be given by $\psi(\xi)=\int_{\mathbb{R}}(1-\cos (\xi x)) \nu(d x)$, where $\nu$ is a symmetric, infinite Lévy measure. The corresponding Pruitt function $h(r)$ is given by

$$
h(r)=\int_{\mathbb{R}}\left(1 \wedge\left(|x|^{2} r^{-2}\right)\right) \nu(d x), \quad r>0 .
$$

We will assume the following scaling conditions for the function $h$ : for some $0<\alpha \leq$ $\beta \leq 2$ and $0<C_{1} \leq 1 \leq C_{2}<\infty$,

$$
C_{1} \lambda^{-\alpha} h(r) \leq h(\lambda r) \leq C_{2} \lambda^{-\beta} h(r), 0<r \leq 1,0<\lambda \leq 1 .
$$

We claim that the above assumption is equivalent to the following weak scaling property for $\psi$ : there are constants $0<C_{1}^{*} \leq 1 \leq C_{2}^{*}<\infty$,

$$
C_{1}^{*} \lambda^{\alpha} \psi(\xi) \leq \psi(\lambda \xi) \leq C_{2}^{*} \lambda^{\beta} \psi(\xi),|\xi| \geq 1, \lambda \geq 1 .
$$

The argument of the equivalence is postponed to Section 4.

Once the condition (6) (or equivalently (7)) is satisfied, we say that the characteristic exponent $\psi$ (or the Lévy measure $\nu$ ) have the weak scaling property with indices $\alpha, \beta$, and write $\psi \in \mathrm{WSC}(\alpha, \beta)$ (resp. $\nu \in \mathrm{WSC}(\alpha, \beta))$.

By $\psi_{i}, \nu_{i}$ and $h_{i}$ we denote corresponding characteristic exponents, Lévy measures and Pruitt functions of coordinates $Z^{i}$ of the process $Z=\left(Z^{1}, \ldots, Z^{d}\right)$.

We will consider two cases:

(A) All characteristic exponents $\psi_{i}, i=1, \ldots, d$ are equal and $\psi_{1} \in \mathrm{WSC}(\alpha, \beta)$.

(B) Characteristic exponents $\psi_{i}, i=1, \ldots, d$ are not the same and $\psi_{i} \in \mathrm{WSC}(\alpha, \beta), i=$ $1, \ldots, d$. 
In both of these cases, the process $Z$ has the transition density $\widetilde{G}_{t}(x, y)=\widetilde{G}_{t}(y-x)$, where

$$
\widetilde{G}_{t}(w)=\prod_{i=1}^{d} \widetilde{g}_{t}^{i}\left(w_{i}\right), \quad w=\left(w_{1}, \ldots, w_{d}\right) \in \mathbb{R}^{d},
$$

and $\widetilde{g}_{t}^{i}, i=1, \ldots, d$ are the distribution densities for the coordinates (all $\widetilde{g}_{t}^{i}$ are the same in the case $(\mathbf{A}))$.

Next, we assume the following conditions on the coefficients.

(C) For any $t \geq 0, x \in \mathbb{R}^{d} A_{t}(x)=\left(a_{t, i, j}(x)\right)$ is a $d \times d$ matrix and there are constants $C_{3}, \ldots, C_{6}>0, \gamma_{1}, \gamma_{2} \in(0,1]$ such that for any $s, t \geq 0, x, y \in \mathbb{R}^{d}, i, j \in\{1, \ldots, d\}$,

$$
\begin{gathered}
\left|a_{t, i, j}(x)\right| \leq C_{3}, \\
\left|\operatorname{det}\left(A_{t}(x)\right)\right| \geq C_{4}, \\
\left|a_{t, i, j}(x)-a_{t, i, j}(y)\right| \leq C_{5}|x-y|^{\gamma_{1}}, \\
\left|a_{s, i, j}(x)-a_{t, i, j}(x)\right| \leq C_{6}|s-t|^{\gamma_{2}} .
\end{gathered}
$$

The function $\left((0, \infty) \times \mathbb{R}^{d} \times \mathbb{R}^{d}\right) \ni(t, x, z) \rightarrow U_{t}(x, z) \in \mathbb{R}^{d}$ is continuous and there are constants $C_{7}>0$ and

$$
\gamma_{3}>\max (1, \beta)
$$

such that for any $t \geq 0, x, z \in \mathbb{R}^{d}$

$$
\left|U_{t}(x, z)\right| \leq C_{7}|z|^{\gamma_{3}} .
$$

In the case (A), the Hölder indices $\gamma_{1}, \gamma_{2}$ can be arbitrarily small. In the case (B), these indices and the $U$-smallness index $\gamma_{3}$ should satisfy certain additional assumptions. Namely, we assume the following

$$
\frac{\beta}{\alpha}<1+\gamma_{1}, \quad \frac{1}{\alpha}-\frac{1}{\beta}<\gamma_{2}, \quad \frac{\beta}{\alpha}<\gamma_{3} .
$$

For abbreviation, for any $u>0$ we will use the notation

$$
\kappa(u)=\left(u, h_{1}(1), \ldots, h_{d}(1), h_{1}^{-1}(1), \ldots, h_{d}^{-1}(1), h_{1}^{-1}(1 / u), \ldots, h_{d}^{-1}(1 / u)\right) .
$$

2.2. Main statements. In this section, we formulate the main statements of the paper.

Theorem 2.1. Assume either $(\boldsymbol{A}),(\boldsymbol{C})$, or $(\boldsymbol{B}),(\boldsymbol{C}),(\boldsymbol{D})$. Then for any $x \in \mathbb{R}^{d}$ the $S D E$ (1) has a unique weak solution $X$. The process $X$ is a time-inhomogeneous Markov process which has a transition density $p_{t, s}(x, y)$. The transition density admits a representation

$$
p_{t, s}(x, y)=\widetilde{p}_{t, s}(x, y)+\widetilde{r}_{t, s}(x, y), \quad x, y \in \mathbb{R}^{d}, \quad 0 \leq t<s,
$$

where $\widetilde{p}_{t, s}(x, y)$ is given by (4) and the residual part $\widetilde{r}_{t, s}(x, y)$ satisfies

$$
\int_{\mathbb{R}^{d}}\left|\widetilde{r}_{t, s}(x, y)\right| d y \leq c(s-t)^{\varepsilon_{0}}, \quad x \in \mathbb{R}^{d},
$$

where $\varepsilon_{0}$ is defined in Remark 5.1 and the constant $c$ depends only on $d, \alpha, \beta, \gamma_{1}, \gamma_{2}, \gamma_{3}$, $C_{1}, \ldots, C_{7}, h_{1}(1), \ldots, h_{d}(1)$.

Theorem 2.1 actually states that the distribution density for $X_{s}$ conditioned by $X_{t}=x$ can be approximated by the density of the variable (5), with the error of approximation given in the integral form. A natural question would be to obtain other types of the bounds for the residue, e.g. uniform in $x, y$. It is known that, in the essentially singular setting, the residue can be locally unbounded, see Example 2.5 below. Hence, in order to get a uniform bound for the residue, one has to impose some new intrinsic assumptions. Here we give one such assumption, formulated in the form inspired by the change of measure 
argument used in [30]. Alternative possibility would be to use a certain integral-in- $x$ condition, similar to (3.17) in [31] or (3.17) - (3.19) in [22].

Denote by $\mu$ the Lévy measure of the process $Z$, and define

$$
T^{t, z} f(x)=f\left(x+V_{t}(x, z)\right) .
$$

Assume the following.

(I) For all $t$ and $\mu$-a.a. $z, T^{t, z}$ is a bounded linear operator in $L_{1}\left(\mathbb{R}^{d}\right)$, and there exists $C_{8}<\infty$ such that

$$
\left\|T^{t, z}\right\|_{L_{1} \rightarrow L_{1}} \leq C_{8}, \quad t \geq 0, \quad z \in \operatorname{supp} \mu .
$$

We have the following representation of the transition density.

Theorem 2.2. Let the conditions of Theorem 2.1 and additional assumption (I) hold. Then the density $p_{t, s}(x, y)$ is bounded, that is

$$
\sup _{x, y \in \mathbb{R}^{d}} p_{t, s}(x, y)<\infty, \quad 0 \leq t<s<\infty .
$$

Moreover, for any $\tau>0$ there exists $c>0$, depending only on $d, \alpha, \beta, \gamma_{1}, \gamma_{2}, \gamma_{3}$, $C_{1}, \ldots, C_{8}, \kappa(\tau)$ such that the residual term in the representation (15) satisfies

$$
\left|\widetilde{r}_{t, s}(x, y)\right| \leq c \widetilde{G}_{s-t}(0)(s-t)^{\varepsilon_{0}}, \quad 0<s-t \leq \tau, \quad x, y \in \mathbb{R}^{d} .
$$

In particular, the following two-sided on-diagonal estimate for $p_{t, s}(x, y)$ holds: for $0<s-t \leq \tau, x \in \mathbb{R}^{d}$,

$$
\frac{1}{\left|\operatorname{det} A_{t}(x)\right|}\left(1-c(s-t)^{\varepsilon_{0}}\right) \leq \frac{p_{t, s}(x, x)}{\widetilde{G}_{s-t}(0)} \leq \frac{1}{\left|\operatorname{det} A_{t}(x)\right|}\left(1+c(s-t)^{\varepsilon_{0}}\right) \text {. }
$$

Define by $\left\{P_{t, s}\right\}$ the evolutionary family corresponding to the process $X$ in the usual way: for any $0 \leq t<s, x \in \mathbb{R}^{d}$ and a bounded Borel function $f: \mathbb{R}^{d} \rightarrow \mathbb{R}$,

$$
P_{t, s} f(x)=\int_{\mathbb{R}^{d}} p_{t, s}(x, y) f(y) d y .
$$

Under just the basic conditions of Theorem 2.1, we prove Hölder continuity of this evolutionary family.

Theorem 2.3. Assume either (A),(C), or (B),(C),(D). For any $0<\gamma<\gamma^{\prime}<\alpha$, $\gamma \leq 1,0<s-t \leq \tau, x, y \in \mathbb{R}^{d}$ and a bounded Borel function $f: \mathbb{R}^{d} \rightarrow \mathbb{R}$ we have

$$
\left|P_{t, s} f(x)-P_{t, s} f(y)\right| \leq c|x-y|^{\gamma}(s-t)^{-\gamma^{\prime} / \alpha}\|f\|_{\infty}, \quad 0 \leq t<s<\infty,
$$

where $c$ depends only on $\gamma, \gamma^{\prime}, d, \alpha, \beta, \gamma_{1}, \gamma_{2}, \gamma_{3}, C_{1}, \ldots, C_{7}, \kappa(\tau)$.

2.3. Examples. Let us give several examples illustrating various specific issues of the model. Our first example shows that the distributions of the components of the Lévy noise $Z$ can be quite singular. Note that a simplest example of a Lévy measure $\nu \in \operatorname{WSC}(\alpha, \beta)$ is a symmetric $\alpha$-stable Lévy measure

$$
\nu(d x)=c \frac{d x}{|x|^{\alpha+1}},
$$

for which

$$
h(r)=\frac{4 c}{\alpha(2-\alpha)} r^{-\alpha}
$$

and thus (6) holds true with $\beta=\alpha$ and $C_{1}=C_{2}=1$. The weak scaling property has the same spirit with the (true) scaling property of the $\alpha$-stable Lévy measure, but is much more flexible. 
Example 2.4. (Discretized $\alpha$-stable measure) Let $\mu(d x)$ be obtained from the symmetric $\alpha$-stable measure (17) by discretization in the following way:

$$
\mu(d x)=\sum_{k=1}^{\infty} \frac{\nu\left(\left\{y: \rho_{k+1}<|y| \leq \rho_{k}\right\}\right)}{2}\left(\delta_{-\rho_{k}}(d x)+\delta_{\rho_{k}}(d x)\right),
$$

where $\rho_{k} \searrow 0$ is a given sequence. Assume that $\left\{\rho_{k}\right\}$ decays not faster than geometrically; that is, for some $c>0$

$$
\rho_{k+1} \geq c \rho_{k}, \quad k \geq 1 .
$$

Then it is easy to show that the Pruitt function for $\mu$ satisfies

$$
B_{1} r^{-\alpha} \leq h(r) \leq B_{2} r^{-\alpha}, \quad r \in(0,1],
$$

for the reader's convenience we prove this inequality in Appendix $\mathrm{A}$ below. This inequality yields immediately that the discretized measure $\mu$ belongs to the same class $\operatorname{WSC}(\alpha, \alpha)$ with the original $\alpha$-stable measure.

The following two examples illustrate the difference between the integral-in-y estimate for the residual term $r_{t, s}(x, y)$ from Theorem 2.1 and the uniform estimate for this term from Theorem 2.2. First, we note that, under just the basic assumptions of Theorem 1 . the transition probability density may be locally unbounded.

Example 2.5. (See [30, Remark 4.23], [22, Example 4.2]). Let $d>1$, all the coordinates $Z^{i}, i=1, \ldots, d$ have the same $\alpha$-stable distribution, and $V_{t}(x, z)=A(x) z$, where the matrices $A(x)$ are Hölder continuous in $x$, for each $x \in \mathbb{R}^{d}$ the matrix $A(x)$ is a rotation (hence, an isometry) and for any $x$ in some open cone with vertex at 0 , which satisfies $|x| \geq 1$ we have $A(x) \mathbf{e}_{1}=x /|x|$. Then for $\alpha+1 \leq d$, for any $x \in \mathbb{R}^{d}$ the transition probability density $p_{t}(x, y)$ is unbounded at any neighbourhood of the point $y=0$.

In the above example, an 'accumulation of mass' effect appears due to singularity of the noise combined with a non-trivial rotation provided by the matrix $A(x)$. The next example shows a typical situation where the additional condition (I) holds true, and thus the 'accumulation of mass' effect does not appear.

Example 2.6. Let the function $V_{t}(x, z)$ be Lipschitz continuous in $x$ with the Lipshitz constant satisfying

$$
\operatorname{Lip}\left(V_{t}(\cdot, z)\right) \leq \rho, \quad t \geq 0, \quad z \in \mathbb{R}^{d}
$$

where $\rho<1$. Then the mapping $I_{\mathbb{R}^{d}}+V_{t}(\cdot, z)$ has an inverse and

$$
\operatorname{Lip}\left(\left[I_{\mathbb{R}^{d}}+V_{t}(\cdot, z)\right]^{-1}\right) \leq \frac{1}{1-\rho}, \quad t \geq 0, \quad z \in \mathbb{R}^{d} .
$$

Moreover $\left[I_{\mathbb{R}^{d}}+V_{t}(\cdot, z)\right]^{-1}$ has a gradient, which is defined a.e. with respect to the Lebesgue measure and bounded, see [7]. In addition, the following change of variables formula holds [18]:

$$
\int_{\mathbb{R}^{d}} f\left(x+V_{t}(x, z)\right) d x=\int_{\mathbb{R}^{d}} f(v) \operatorname{det}\left(\nabla_{v}\left[I_{\mathbb{R}^{d}}+V_{t}(v, z)\right]^{-1}\right) d v .
$$

This yields (I) with

$$
C_{8}=\sup _{t, z} \operatorname{esssup}_{v}\left|\operatorname{det}\left(\nabla_{v}\left[I_{\mathbb{R}^{d}}+V_{t}(v, z)\right]^{-1}\right)\right| \leq \frac{d !}{(1-\rho)^{d}} .
$$

Our last example explains why in the case (B), i.e. for a cylindrical noise which has different scaling indices of the coordinates, non-trivial assumptions on the Hölder indices of the coefficients should be made, on the contrary to the case (A), where the Hölder indices can be arbitrarily small. 
Example 2.7. Let $Z^{i}, i=1, \ldots, d$ be symmetric $\alpha_{i}$-stable with different $\alpha_{i}, i=1, \ldots, d$. The process $Z=\left(Z^{1}, \ldots, Z^{d}\right)$ fits to our case (B) with $\alpha=\min _{i} \alpha_{i}, \beta=\max _{i} \alpha_{i}$. In this example, we show that in such - extremely spatially non-homogeneous - setting the additional assumption (D) is crucial in the sense that, without this condition, the structure of the transition density can be quite different.

Take $d=2$ and $\alpha_{1}=\alpha<\alpha_{2}=\beta$. Take also $V_{t}(x, z)=A_{t} z$, where

$$
A_{t}=\left(\begin{array}{cc}
1 & t^{\gamma} \\
t^{\gamma} & 1
\end{array}\right)
$$

is a matrix-valued function which depends on $t$, only. Then the additional assumption (I) holds true since each operator $T^{t, z}$ is just an isometry which corresponds to the shift of the variable $x \mapsto x+A_{t} z$ (we can also refer to Example 2.6 here). Denote, as usual, $f \asymp g$ if the ratio $\frac{f}{g}$ is bounded and separated from 0 . Then, by Theorem 2.2, one has

$$
p_{t, s}(x, x) \asymp \widetilde{G}_{s-t}(0) \asymp(s-t)^{-\frac{1}{\alpha}-\frac{1}{\beta}}
$$

provided that $\frac{1}{\alpha}-\frac{1}{\beta}<\gamma$, which is actually the second inequality in (14) (the first and the third one hold true automatically). For $\frac{1}{\alpha}-\frac{1}{\beta}>\gamma$ the situation changes drastically; namely, we have

$$
p_{0, s}(x, x) \leq C s^{-\gamma-\frac{2}{\beta}} \quad \text { and } \quad \frac{s^{-\gamma-\frac{2}{\beta}}}{s^{-\frac{1}{\alpha}-\frac{1}{\beta}}} \rightarrow 0, \quad s \rightarrow 0+.
$$

We prove this relation in Appendix $\mathrm{A}$, here we give an informal explanation of the effect. The original noise has two components, a 'weaker' one and a 'stronger' one, which act along the 1 st and the 2 nd coordinate vectors $\mathbf{e}_{1}, \mathbf{e}_{2}$, respectively. The law of the solution to SDE (11) with $x=0, t=0$ is a convolution of the laws of the solutions $X^{(1)}, X^{(2)}$ to SDE (1), where instead of $Z$ we substitute these two components of the noise separately. Consider the projections of these laws on the direction $\mathbf{e}_{1}$, the one where the 'weaker' noise acts. It is easy to show that the projection of the law of $X_{s}^{(1)}$ on $\mathbf{e}_{1}$ has a distribution density $p_{0, s}^{(1,1)}(0, \cdot)$ with $p_{0, s}^{(1,1)}(0,0) \asymp s^{-\frac{1}{\alpha}}$. On the other hand, any jump of the noise at the time $t$, having the altitude $z$ and the direction $\mathbf{e}_{2}$, produces a jump of the 1st coordinate with the altitude $t^{\gamma} z$. Then it is not difficult to prove (and it is easy to believe) that the projection of the law of $X_{s}^{(2)}$ on $\mathbf{e}_{1}$ has a distribution density $p_{0, s}^{(2,1)}(0, \cdot)$ with $p_{0, s}^{(2,1)}(0,0) \asymp s^{-\gamma-\frac{1}{\beta}}$. Since $-\gamma-\frac{1}{\beta}>-\frac{1}{\alpha}$, this means that the projection of the law of $X_{s}^{(1)}$ in the direction $\mathbf{e}_{1}$ is 'more concentrated' around 0 than the same projection for the law of $X_{s}^{(2)}$. The direction $\mathbf{e}_{1}$ is the 'worst possible' here in the sense that $A_{0} \mathbf{e}_{1}$ is equal to the first basis vector $\mathbf{e}_{1}$ and is orthogonal to the second one $\mathbf{e}_{2}$. One can actually show that the same 'concentration comparison' hold true for the projections on arbitrary direction 1 . This means that, in the convolution of the laws $X^{(1)}, X^{(2)}$, the first component is negligible when compared to the second one. It should be noted that, in this example, the one-dimensional noise $Z^{2} \mathbf{e}_{2}$ generates a two-dimensional distribution density, which is actually a hypoellipticity-type effect. This density appears to be principal for the entire solution, which indicates that, without a condition of the type (D), analysis of the SDE with different components of the cylindrical noise should involve a study of hypoellipticity features. A systematic study of that type does not seem realistic for SDEs with low regularity of the coefficients, thus we restrict ourselves to the case where the condition (D) holds and thus hypoellipticity-type effects do not come into play.

2.4. Literature overview. Our main tool in the construction of the heat kernel $p_{t, s}(x, y)$ of the solution to SDE (90) is the parametrix method, properly adapted to the sophisticated model we have. The parametrix method was first proposed by Levi [33], Hadamard [17] and Gevrey [13] for differential operators and later extended by Feller [11] to a simple non-local 
setting. The first version of the parametrix method for non-local operators was developed by Kochubei [24, see also the monograph by Eidelman, Ivasyshen \& Kochubei 9]. This method required the Lévy measure of the noise to be comparable with the rotationally invariant $\alpha$-stable Lévy measure, and $\alpha>1$, i.e. the non-local part of the generator should dominate - in the order sense - the gradient part. These results have been extended in numerous directions e.g. by Kolokoltsov [25], where the limitation $\alpha>1$ was removed for the operators without a gradient part; see also Chen \& Zhang [5]. The parametrix method for the stable-like case, where the stability index is $x$-dependent, have been developed first by Kolokoltsov [25]; in the papers of Kühn [27, 26] this problem was treated for a wider class of Lévy kernels assuming a kind of sector condition for the symbol of the operators. In Knopova \& Kulik [21, 32] the parametrix method was extended to super-critical case, where the (non-trivial) gradient part is not dominated by an $\alpha$-stable noise with $\alpha<1$. In all these results the Lévy noise, principally, was comparable with the rotationally invariant $\alpha$-stable one. Lévy-type models with other types of the reference measures have been studied as well; see Bogdan, Knopova \& Sztonyk [2, Kulczycki \& Ryznar [29], where $\alpha$-stable reference measures with various types of spherical measure (i.e. the distribution of the jumps directions) have been treated, and Grzywny \& Szczypkowksi [15], where the reference measure is rotationally invariant and satisfies weak scaling condition. The symmetry assumption, typically imposed on the Lévy noise in order to simplify the technicalities, is not substantial; see the recent publications by Chen, $\mathrm{Hu}$, Xie \& Zhang [6, 4], Grzywny \& Szczypkowksi [15], Kulik [31] for the parametrix method for various non-symmetric Lévy-type models.

Essentially singular models, where the distributions of a jump for various starting points are mutually singular, lack a fixed reference measure, to the striking contrast with the results listed above. This leads to a considerably new technical difficulties; essentially singular models also exhibit new effects such as the one discussed in Example 2.5. For the first advances in the study of such models see Kulczycki, Ryznar \& Sztonyk [30, 28] and Knopova, Kulik \& Schilling [22], which we have already mentioned and discussed in the Introduction.

\section{ROAD MAP TO THE PROOFS}

3.1. The parametrix method. We will construct the transition density $p_{t, s}(x, y)$ for the unknown process using a proper modification of the parametrix method, which is a classical analytical method for construction of fundamental solutions to elliptic and parabolic PDEs of second order; for a detailed overview of the history and the ideas the method is based on, we refer to [20] or [22]. Here we outline briefly the construction, taking into account the fact that the actual model is non-homogeneous in time.

Consider a (time-dependent) operator $L_{t}$ with the domain $C_{\infty}^{2}\left(\mathbb{R}^{d}\right)$, given by

$$
\begin{array}{r}
L_{t} f(x)=\mathrm{P} \cdot \mathrm{V} \cdot \int_{\mathbb{R}^{d}}\left(f\left(x+V_{t}(x, z)\right)-f(x)\right) \mu(d z) \\
=\sum_{k=1}^{d} \int_{\mathbb{R}}\left(f\left(x+u A_{t}(x) \mathbf{e}_{k}+U_{t}\left(x, \mathbf{e}_{k} u\right)\right)-f(x)\right. \\
\left.\quad-u 1_{|u| \leq 1} \nabla f(x) \cdot A_{t}(x) \mathbf{e}_{k}\right) \nu_{k}(d u),
\end{array}
$$

where $\mu$ is the Lévy measure of the process $Z, \nu_{k}$ is the Lévy measure of the $k$-th component $Z^{k}, k=1, \ldots, d$, and P.V. means that the first integral is taken in the principal value sense. By the virtue of the Itô formula, one can naturally expect that, once the solution $X$ to (11) is well defined and is a (time-inhomogeneous) Markov process, the operator $L_{t}$ should be its generator. Corresponding Kolmogorov's backward differential equation for the transition 
probability density of $X$ has the form

$$
\left(\partial_{t}+L_{t ; x}\right) p_{t, s}(x, y)=0, \quad 0 \leq t<s, \quad x, y \in \mathbb{R}^{d},
$$

here and below $x$ at the operator $L_{t ; x}$ indicates that the operator $L_{t}$ is applied with respect to the variable $x$. Together with the initial condition

$$
p_{t, s}(x, y) \rightarrow \delta_{x}(y), \quad s \rightarrow t,
$$

this actually gives that $p_{t, s}(x, y)$ is a fundamental solution to the parabolic Cauchy problem for the operator $L_{t}$. The streamline of the method is to construct a (candidate for) the required fundamental solution, and then to show that this kernel $p_{t, s}(x, y)$ indeed corresponds to the unique weak solution to (11).

To construct a candidate for the fundamental solution, we use the parametrix method, which, in a wide generality, can be outlined as follows. Fix a function $p_{t, s}^{(0)}(x, y)$, which is $C^{1}$ in $t$ and $C_{\infty}^{2}\left(\mathbb{R}^{d}\right)$ in $x$ for a fixed $s, y$, and define

$$
q_{t, s}^{(0)}(x, y)=-\left(\partial_{t}+L_{t ; x}\right) p_{t, s}^{(0)}(x, y) .
$$

Then differential equation (20) can be written as

$$
\left(\partial_{t}+L_{t ; x}\right)\left(p_{t, s}(x, y)-p_{t, s}^{(0)}(x, y)\right)=q_{t, s}^{(0)}(x, y) .
$$

Since we expect $p_{t, s}(x, y)$ to be a (true) fundamental solution, we can formally resolve the above equation as

$$
p_{t, s}(x, y)=p_{t, s}^{(0)}(x, y)+\int_{t}^{s} \int_{\mathbb{R}^{d}} p_{t, r}(x, v) q_{r, s}^{(0)}(v, y) d v d r, \quad 0 \leq t<s, \quad x, y \in \mathbb{R}^{d},
$$

The identity (22) can be seen as an integral equation for the unknown kernel $p_{t, s}(x, y)$, which is easier to deal with than the original differential equation (20). This is the essence of the method: we first construct a candidate for the transition probability density $p_{t, s}(x, y)$ as the solution to the integral equation (22) and then study its properties in order to show that this kernel indeed corresponds to the unique weak solution to (11).

3.2. Choice of the zero-order approximation. One of the crucial points in the strategy outlined above is the choice of the kernel $p_{t, s}^{(0)}(x, y)$, which has a natural meaning of the 'zero-order approximation' term for the unknown $p_{t, s}(x, y)$. This choice determines the 'differential error of approximation' $q_{t, s}^{(0)}(x, y)$, and should be precise enough to guarantee integrability of $q_{t, s}^{(0)}(x, y)$; note that we require this integrability in order to treat the integral equation (22) properly. We will choose $p_{t, s}^{(0)}(x, y)$ in the form

$$
p_{t, s}^{(0)}(x, y)=\frac{1}{\left|\operatorname{det} A_{s}(y)\right|} G_{s-t}\left((y-x)\left(A_{s}(y)^{-1}\right)^{T}\right),
$$

where $G_{r}(w)$ is the distribution density of a dynamically truncated Lévy noise; see Section 5 for its definition and properties. The density $G_{s-t}$ is also dependent on $\varepsilon>0$, however we do not reflect this in our notation. Such a choice combines two ideas. The first one is the classical parametrix idea that a good 'zero-order approximation' to the fundamental solution can be obtained by taking the heat kernel for an equation with constant coefficients (e.g. the Gaussian kernel in the diffusion setting) and substituting there the coefficients frozen at the endpoint $y, s$. This classical construction also suggests that negligible (in a sense) parts should be removed from the generator: in the diffusion setting this is the drift (gradient) term, in our case this is the non-linear jump term $U_{t}(x, z)$. Though, such classical parametrix construction appears to be not precise enough in the singular Lévy noise setting. Namely, such a construction would suggest, instead of (23), the choice

$$
\widetilde{p}_{t, s}^{(0)}(x, y)=\frac{1}{\left|\operatorname{det} A_{s}(y)\right|} \widetilde{G}_{s-t}\left((y-x)\left(A_{s}(y)^{-1}\right)^{T}\right),
$$


recall that $\widetilde{G}_{t}(\cdot)$ is the distribution density of $Z_{t}$. However, in general, $\widetilde{p}_{t, s}^{(0)}(x, y)$ may provide quite a poor approximation to $p_{t, s}(x, y)$ : e.g. in the model from Example 2.7 it can be verified easily that

$$
\int_{\mathbb{R}^{d}} \widetilde{p}_{t, s}^{(0)}(x, y) d y=\infty
$$

in the striking contrast to the fact that $p_{t, s}(x, \cdot)$ should be a probability density. This is an essentially non-local effect; in order to avoid it we use the second idea to 'cut off' big jumps. In [30, 28, the cut off level was chosen small but fixed, which required Lipschitz continuity of the coefficients. In [22], a time dependent cut off level was proposed, which allows one to treat the models where the coefficients are only assumed to be Hölder continuous. Here we use the same dynamic truncation idea, properly adapted to the current model. Namely, $G_{r}(w)$ in (23) will be the distribution density of $\widehat{Z}_{r}=\left(\widehat{Z}_{r}^{1}, \ldots, \widehat{Z}_{r}^{d}\right)$, where the components are independent and

$$
\widehat{Z}_{r}^{i}=\int_{0}^{r} \int_{|u| \leq R_{\rho}^{(i)}} u N^{i}(d \rho, d u)
$$

where $N^{i}(d \rho, d u)$ is the Poisson point measure corresponding to $Z^{i}$, and the time-dependent truncation function $R_{\rho}^{(i)}=R_{\rho}^{(i)}(\varepsilon)$ is determined by means of the corresponding Pruitt function $h_{i}(r)$ and $\varepsilon>0$ of our choice. Note that in the case (A) the cut off level is the same for all coordinates, while in the case (B) these levels can be quite different. This is the actual reason for the condition (D) to appear in the case (B): we will need this condition in order to balance, in a sense, the 'cut off effects' for various coordinates.

3.3. Functional analytical framework. It is convenient to treat (22) within the functional analytic framework introduced in [22, Section 5.2], properly adapted to the time non-homogeneous setting. Consider the Banach space $L^{\infty}(d x) \otimes L^{1}(d y)$ of kernels $k(x, y)$ satisfying

$$
\|k\|_{\infty, 1}:=\operatorname{esssup}_{x \in \mathbb{R}^{d}} \int_{\mathbb{R}^{d}}|k(x, y)| d y<\infty .
$$

Each kernel $k \in L^{\infty}(d x) \otimes L^{1}(d y)$ generates a bounded linear operator $K$ in the space $B_{b}=B_{b}\left(\mathbb{R}^{d}\right)$ of bounded measurable functions,

$$
K f(x)=\int_{\mathbb{R}^{d}} k(x, y) f(y) d y, \quad f \in B_{b}\left(\mathbb{R}^{d}\right),
$$

with the operator norm $\|K\|_{B_{b} \rightarrow B_{b}}$ equal to the norm $\|k\|_{\infty, 1}$. Denote $P_{t, s}, P_{t, s}^{(0)}, Q_{t, s}^{(0)}, 0<$ $t<s$ the families of operators corresponding to the unknown transition probability density $p_{t, s}(x, y)$ and the kernels $p_{t, s}^{(0)}(x, y), q_{t, s}^{(0)}(x, y)$ introduced above. Then (22) can be equivalently written as

$$
P_{t, s}=P_{t, s}^{(0)}+\int_{t}^{s} P_{t, r} Q_{r, s}^{(0)} d r, \quad 0 \leq t<s .
$$

Let $0<\varepsilon \leq \varepsilon_{0}$, with $\varepsilon_{0}$ defined in Remark 5.1. In the whole subsection $c$ denotes a constant dependent on $d, \alpha, \beta, \gamma_{1}, \gamma_{2}, \gamma_{3}, C_{1}, \ldots, C_{8}, \kappa(\tau)$ and $\varepsilon$. In Lemma 5.10 below we prove an estimate for $q_{t, s}^{(0)}(x, y)$ in $\|\cdot\|_{\infty, 1}$-norm which actually can be written as a bound for the operator norm

$$
\left\|Q_{t, s}^{(0)}\right\|_{B_{b} \rightarrow B_{b}} \leq c(s-t)^{-1+\varepsilon}, \quad 0<s-t \leq \tau .
$$

This allows us to treat (24), in a standard way, as a Volterra equation with a mild (integrable) singularity. Recall that each kernel $p_{t, s}(x, y)$ is supposed to be a probability density, hence it is necessary that

$$
\left\|P_{t, s}\right\|_{B_{b} \rightarrow B_{b}}<\infty
$$


The unique solution to (24) which satisfies (26) can be interpreted as a classical Neumann series

$$
\begin{aligned}
P_{t, s} & =P_{t, s}^{(0)}+\sum_{k=1}^{\infty} \int_{t<r_{1}<\cdots<r_{k}<s} \ldots \int_{t, r_{1}}^{(0)} Q_{r_{1}, r_{2}}^{(0)} \ldots Q_{r_{k}, s}^{(0)} d r_{1} \ldots d r_{k} \\
& =P_{t, s}^{(0)}+\int_{t}^{s} P_{t, r}^{(0)} Q_{r, s} d r
\end{aligned}
$$

where the operator

$$
Q_{t, s}:=Q_{t, s}^{(0)}+\sum_{k=1}^{\infty} \int_{t<r_{1}<\cdots<r_{k}<s} \ldots \int_{t, r_{1}}^{(0)} \ldots Q_{r_{k}, s}^{(0)} d r_{1} \ldots d r_{k}
$$

corresponds to the kernel

$$
q_{t, s}(x, y):=\sum_{k=0}^{\infty} q_{t, s}^{(k)}(x, y), \quad q_{t, s}^{(k+1)}(x, y):=\int_{t}^{s} \int_{\mathbb{R}^{d}} q_{t, r}^{(k)}(x, v) q_{r, s}^{(0)}(v, y) d v d r, \quad k \geq 0 .
$$

The series (28), (29) converge uniformly in $0<s-t \leq \tau$ in the operator norm $\|\cdot\|_{B_{b} \rightarrow B_{b}}$ and the norm $\|\cdot\|_{\infty, 1}$, respectively. This follows easily from (25), since for $k \geq 1$

$$
\begin{aligned}
\left\|q_{t, s}^{(k)}\right\|_{\infty, 1} & =\left\|\int_{t<r_{1}<\cdots<r_{k}<s} \ldots \int_{t_{, r_{1}}} \ldots Q_{r_{k}, s}^{(0)} d r_{1} \ldots d r_{k}\right\|_{B_{b} \rightarrow B_{b}} \\
& \leq \int_{t<r_{1}<\cdots<r_{k}<s} \ldots \int_{t, r_{1}}\left\|Q_{r_{k}, s}^{(0)}\right\|_{B_{b} \rightarrow B_{b}} d r_{1} \ldots d r_{k} \\
& \leq c^{k} \int_{t<r_{1}<\cdots<r_{k}<s} \ldots \int_{1}\left(r_{1}-t\right)^{-1+\epsilon} \cdot \ldots \cdot\left(s-r_{k}\right)^{-1+\epsilon} d r_{1} \ldots d r_{k} \\
& =(s-t)^{-1+k \epsilon} \frac{(c \Gamma(\epsilon))^{k}}{\Gamma(k \epsilon)},
\end{aligned}
$$

and the Gamma function $\Gamma(z)$ behaves asymptotically like $\sqrt{2 \pi} z^{z-\frac{1}{2}} e^{-z} \gg c^{z}$ as $z \rightarrow \infty$. This estimate yields

$$
\left\|q_{t, s}\right\|_{\infty, 1} \leq c(s-t)^{-1+\epsilon}, \quad 0<s-t \leq \tau .
$$

In Lemma 5.8 below we prove that $p_{t, s}^{(0)}(x, y)$ is bounded in $\|\cdot\|_{\infty, 1}$-norm, which similarly to (30) yields that the residue $r_{t, s}(x, y)=p_{t, s}(x, y)-p_{t, s}^{(0)}(x, y)$ satisfies

$$
\left\|r_{t, s}\right\|_{\infty, 1} \leq c(s-t)^{\epsilon}, \quad 0<s-t \leq \tau .
$$

These representations and estimates form an essential part of the proof of Theorem 2.1.

3.4. Approximate fundamental solution and weak uniqueness of the solution. Let $C_{\infty}\left(\mathbb{R}^{d}\right)$ denote a space of continuous functions vanishing at infinity. Define $P_{t, t}=$ $P_{t, t}^{(0)}=\mathrm{id}$, the identity operator. The operator families $\left\{P_{t, s}^{(0)}\right\},\left\{Q_{t, s}^{(0)}\right\}$ have the following properties, see Lemmas 5.8, 5.10, 5.13, 5.15 and 5.16,

Lemma 3.1. Each of the operators $P_{t, s}^{(0)}, 0 \leq t \leq s, Q_{t, s}^{(0)}, 0 \leq t<s$ maps $C_{\infty}\left(\mathbb{R}^{d}\right)$ to $C_{\infty}\left(\mathbb{R}^{d}\right)$. The corresponding families of operators are strongly continuous w.r.t. $t, s$.

Note that the operator norm $\|\cdot\|_{C_{\infty} \rightarrow C_{\infty}}$ is dominated by the norm $\|\cdot\|_{B_{b} \rightarrow B_{b}}$, thus the norm estimates from the previous section yield that the series (27) converges in the norm $\|\cdot\|_{C_{\infty} \rightarrow C_{\infty}}$ uniformly in $0 \leq s-t \leq \tau$, and the series (28) converges uniformly for $\tau_{1} \leq s-t \leq \tau$, for any $0<\tau_{1}<\tau$. Moreover, a standard argument based on the strong 
continuity of $Q_{t, s}^{(0)}, t<s$ and the estimate (25) shows that for every $n \geq 1$ the operators $Q_{t, s}^{(n)}$ (defined by kernels $\left.q_{t, s}^{(n)}(x, y)\right)$ are strongly continuous w.r.t. $t<s$. This yields

Corollary 3.2. Each of the operators $P_{t, s}, 0 \leq t \leq s, Q_{t, s}, 0 \leq t<s$ maps $C_{\infty}\left(\mathbb{R}^{d}\right)$ to $C_{\infty}\left(\mathbb{R}^{d}\right)$. Corresponding families of operators are strongly continuous w.r.t. $t, s$.

In general, it might be quite difficult to prove that the kernel $p_{t, s}(x, y)$, constructed as a solution to the integral equation (22), solves the differential equation (20). We avoid this complicated step, using the following approximate procedure. Define for $\eta>0$

$$
P_{t, s, \eta}=P_{t, s+\eta}^{(0)}+\int_{t}^{s} P_{t, r+\eta}^{(0)} Q_{r+\eta, s+\eta} d r, \quad 0 \leq t \leq s .
$$

The following lemma shows that $p_{t, s}(x, y)$ solves the backward Kolmogorov equation (20) in a certain approximate sense.

Lemma 3.3. Let $\tau>0$ and a compact subset $F \subset C_{\infty}\left(\mathbb{R}^{d}\right)$ be fixed.

a)

$$
\left\|P_{t, s, \eta} f-P_{t, s} f\right\| \rightarrow 0, \quad \eta \rightarrow 0
$$

uniformly in $0 \leq s \leq t \leq \tau, f \in F$,

b) For any $f \in C_{0}\left(\mathbb{R}^{d}\right)$ and $\eta>0$, the function $P_{t, s, \eta} f(x)$ is $C^{1}$ in $t$ and $C_{0}^{2}\left(\mathbb{R}^{d}\right)$ in $x$ on $[0, s] \times \mathbb{R}^{d}$, and thus the operators

$$
\Delta_{t, s, \eta}=\left(\partial_{t}+L_{t}\right) P_{t, s, \eta}, \quad s \leq t, \eta>0
$$

are well defined. These operators satisfy

$$
\left\|\Delta_{t, s, \eta} f\right\| \rightarrow 0, \quad \eta \rightarrow 0
$$

uniformly in $s-t>\tau_{1}, 0 \leq s \leq \tau, f \in F$ for any $\tau_{1}>0$ and

$$
\int_{t}^{\tau}\left\|\Delta_{t, r, \eta} f\right\| d r \rightarrow 0, \quad \eta \rightarrow 0
$$

uniformly in $0 \leq t \leq \tau, f \in F$.

The proof of this statement is remarkably simple, and requires only representation (24) and the continuity properties stated in Lemma 3.1 and Corollary 3.2. Thus we give it here.

Proof. Statement a) follows directly from the representation (24) and the continuity properties (Lemma 3.1 and Corollary 3.2).

To prove (33), note that

$$
\begin{aligned}
\Delta_{t, s, \eta} f & =\left(\partial_{t}+L_{t}\right) P_{t, s+\eta}^{(0)} f+\int_{t}^{s}\left(\partial_{t}+L_{t}\right) P_{t, r+\eta}^{(0)} Q_{r+\eta, s+\eta} f d r-P_{t, t+\eta} Q_{t+\eta, t+\eta} f \\
& =Q_{t, s+\eta}^{(0)} f+\int_{t}^{s} Q_{t, r+\eta}^{(0)} Q_{r+\eta, s+\eta} f d r-P_{t, t+\eta} Q_{t+\eta, s+\eta} f \\
& =Q_{t, s+\eta} f-\int_{t}^{t+\eta} Q_{t, r}^{(0)} Q_{r, s+\eta} f d r-P_{t, t+\eta} Q_{t+\eta, s+\eta} f
\end{aligned}
$$

in the last identity we have used that $Q_{t, s}$ is given by (28) and thus satisfies

$$
Q_{t, s}=Q_{t, s}^{(0)}+\int_{t}^{s} Q_{t, r}^{(0)} Q_{r, s} d r .
$$

By the continuity properties Lemma 3.1 a),

$$
\left\|Q_{t, s+\eta} f-P_{t, t+\eta} Q_{t+\eta, s+\eta} f\right\| \rightarrow 0, \quad \eta \rightarrow 0
$$


uniformly in $s-t>\tau, f \in F$. Convergence

$$
\left\|\int_{t}^{t+\eta} Q_{t, r}^{(0)} Q_{r, s+\eta} f d r\right\| \rightarrow 0, \quad \eta \rightarrow 0
$$

follows by the bounds (25), (31). The same bounds combined with (33) yield (34).

Lemma 3.3 provides an efficient tool for identifying weak solutions to the SDE (1). Note that it is easy to prove existence of a weak solution to (11) by smooth approximation of the coefficients and using the compactness argument; see [21, Section 5] for such an argument explained in details. To identify a weak solution to (1) with given initial condition, we will consider operator $\mathcal{L}$ defined by

$$
\mathcal{L} \phi(t, x)=\partial_{t} \phi(t, x)+L_{t ; x} \phi(t, x), \quad \phi \in \mathcal{D}
$$

on the set $\mathcal{D}=C_{\infty}^{1,2}\left([0, \tau] \times \mathbb{R}^{d}\right)$ of functions $\phi(t, x)$ which are $C^{1}$ in $t, C^{2}$ in $x$, and have their derivatives continuous and being from the class $C_{\infty}\left(\mathbb{R}^{d}\right)$ for any $t$ fixed; $\tau>0$ here is a fixed number. The Itô formula yields that, for a weak solution $X$ to SDE (1) with $X_{0}=x$ and $\phi \in \mathcal{D}$, the process

$$
\phi\left(s, X_{s}\right)-\int_{0}^{s} \mathcal{L} \phi\left(r, X_{r}\right) d r, \quad s \in[0, \tau]
$$

is a martingale. We can use, with minor changes, the argument from [31, Section 5.3] to derive from this finite-dimensional distributions of $X$. Namely, let $f \in C_{\infty}\left(\mathbb{R}^{d}\right), \tau>0$ be fixed. Taking $\phi(t, x)=P_{t, \tau, \eta} f(x)$, we get for any $t \leq s \leq \tau$

$$
\begin{aligned}
\mathbb{E}\left[P_{\tau, \tau, \eta} f\left(X_{\tau}\right) \mid \mathcal{F}_{s}\right]-P_{s, \tau, \eta} f\left(X_{s}\right) & =\mathbb{E}\left[\int_{s}^{\tau}\left(\partial_{r}+L_{x}^{r}\right) P_{t, \tau, \eta} f\left(X_{r}\right) d r \mid \mathcal{F}_{s}\right] \\
& =\left[\mathbb{E} \int_{s}^{\tau} \Delta_{t, \tau, \eta} f\left(X_{r}\right) d r \mid \mathcal{F}_{s}\right] .
\end{aligned}
$$

Then by Lemma 3.3, passing to the limit $\eta \rightarrow 0$, we get

$$
\mathbb{E}\left[P_{s, \tau, \eta} f\left(X_{\tau}\right) \mid \mathcal{F}_{s}\right]-P_{s, \tau} f\left(X_{s}\right)=0, \quad s \in[0, \tau]
$$

or, equivalently,

$$
\mathbb{E}\left[f\left(X_{\tau}\right) \mid \mathcal{F}_{s}\right]=P_{s, \tau} f\left(X_{s}\right)
$$

for any $f \in C_{\infty}\left(\mathbb{R}^{d}\right)$ and any pair of time moments $\tau \geq s \geq 0$. Since

$$
P_{t, s} f(x)=\int_{\mathbb{R}^{d}} f(y) p_{t, s}(x, y) d y,
$$

this yields the identity

$$
\mathbf{P}\left(X_{s_{1}} \in A_{1}, \ldots, X_{s_{k}} \in A_{k}\right)=\iint_{A_{1} \times \cdots \times A_{k}} p_{0, s_{1}}\left(x, v_{1}\right) \ldots p_{s_{k-1}, s_{k}}\left(v_{k-1}, v_{k}\right) d v_{1} \ldots d v_{k}
$$

valid for any $k \geq 1, t<s_{1}<\cdots<s_{k}$ and Borel measurable $A_{1}, \ldots, A_{k}$. This identifies uniquely the finite-dimensional distributions of $X$ and proves that $X$ is a (time nonhomogeneous) Markov process with the transition density $p_{t, s}(x, y)$.

3.5. Outline of the rest of the proofs. Recall that our choice of $p_{t, s}^{(0)}(x, y)$ and $q_{t, s}^{(0)}(x, y)$ was dependent on $\varepsilon \in\left(0, \varepsilon_{0}\right]$. In this subsection we choose $\varepsilon=\varepsilon_{0}$.

To complete the proof of Theorem 2.1, we have to prove the bound on the residual term $\widetilde{r}_{t, s}(x, y)$ in the decomposition (15). To do this, we use the decomposition

$$
p_{t, s}(x, y)=p_{t, s}^{(0)}(x, y)+r_{t, s}(x, y)
$$


and the bound (32) for the residual term $r_{t, s}(x, y)$, obtained by the parametrix method. Then

$$
\widetilde{r}_{t, s}(x, y)=p_{t, s}^{(0)}(x, y)-\widetilde{p}_{t, s}(x, y)+r_{t, s}(x, y),
$$

and the required bound follows from the estimate

$$
\left\|p_{t, s}^{(0)}(x, y)-\widetilde{p}_{t, s}(x, y)\right\|_{\infty, 1} \leq c(s-t)^{\epsilon_{0}}, \quad 0<s-t \leq \tau,
$$

which we prove in Lemma 5.18 below. Next, combining (32) and (35) we obtain

$$
\left\|\widetilde{r}_{t, s}(x, y)\right\|_{\infty, 1} \leq c(s-t)^{\epsilon_{0}}, \quad 0<s-t \leq \tau,
$$

Here the constant $c$ is a constant dependent on $d, \alpha, \beta, \gamma_{1}, \gamma_{2}, \gamma_{3}, C_{1}, \ldots, C_{8}, \kappa(\tau)$, but for $\tau \leq \tau_{0}$, where $\tau_{0}$ is defined at the beginning of Section 5 , the constant depends on $d, \alpha, \beta$, $\gamma_{1}, \gamma_{2}, \gamma_{3}, C_{1}, \ldots, C_{8}, h_{1}(1), \ldots, h_{d}(1)$. In fact (36) holds for all $0 \leq t<s$ with a constant dependent on $d, \alpha, \beta, \gamma_{1}, \gamma_{2}, \gamma_{3}, C_{1}, \ldots, C_{8}, h_{1}(1), \ldots, h_{d}(1)$. Indeed, if $s-t>\tau_{0}$ then

$$
\left\|\widetilde{r}_{t, s}(x, y)\right\|_{\infty, 1}=\left\|p_{t, s}(x, y)-\widetilde{p}_{t, s}(x, y)\right\|_{\infty, 1} \leq 2 \leq \frac{2}{\tau_{0}^{\epsilon_{0}}}(s-t)^{\epsilon_{0}} .
$$

The functional analytic framework from Section 3.3 is quite convenient also for proving the uniform estimates for the residual term, stated in Theorem 2.2, see a detailed discussion in [22, Section 5.2]. Namely, the uniform-in- $x, y$ bound for a (continuous) kernel is equivalent to the $\|\cdot\|_{L_{1} \rightarrow B_{b}}$ operator norm for the corresponding integral operator. In addition to the bound (25), we have

$$
\left\|Q_{t, s}^{(0)}\right\|_{L_{1} \rightarrow B_{b}} \leq c G_{s-t}(0)(s-t)^{-1+\varepsilon_{0}}
$$

(Lemma 5.10, estimate (89)). Under the additional assumption (I), we also have

$$
\left\|Q_{t, s}^{(0)}\right\|_{L_{1} \rightarrow L_{1}} \leq c(s-t)^{-1+\varepsilon_{0}}
$$

(Lemma 5.11). Then for any $k \in \mathbb{N}, j=0, \ldots, k$ and $r_{0}<r_{1}<\ldots r_{k+1}$

$$
\begin{aligned}
&\left\|Q_{r_{0}, r_{1}}^{0} \ldots Q_{r_{k}, r_{k+1}}^{0}\right\|_{L_{1} \rightarrow B_{b}} \leq\left\|Q_{r_{0}, r_{1}}^{0}\right\|_{B_{b} \rightarrow B_{b}} \ldots\left\|Q_{r_{j-1}, r_{j}}^{0}\right\|_{B_{b} \rightarrow B_{b}} \\
& \quad \times\left\|Q_{r_{j}, r_{j+1}}^{0}\right\|_{L_{1} \rightarrow B_{b}}\left\|Q_{r_{j+1}, r_{j+2}}^{0}\right\|_{L_{1} \rightarrow L_{1}} \ldots\left\|Q_{r_{k}, r_{k+1}}^{0}\right\|_{L_{1} \rightarrow L_{1}} \\
& \leq c^{k+1} G_{r_{j+1}-r_{j}}(0) \prod_{i=0}^{k}\left(r_{i+1}-r_{i}\right)^{-1+\varepsilon_{0}} .
\end{aligned}
$$

If we take $r_{0}=t, r_{k+1}=t$, and $j$ such that $r_{j+1}-r_{j}=\max _{i}\left(r_{i+1}-r_{i}\right)$, then $G_{r_{j+1}-r_{j}}(0) \leq$

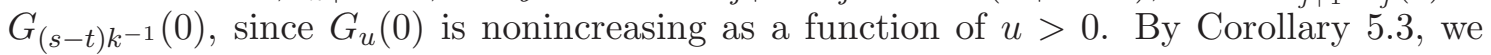
have

Also, by Lemma 4.8 ,

$$
G_{(s-t) k^{-1}}(0) \leq c k^{d / \alpha} G_{s-t}(0), \quad k \in \mathbf{N} .
$$

$$
G_{s-t}(0) \leq c \widetilde{G}_{s-t}(0), 0<s-t \leq \tau .
$$

Then, similarly to (30), (31) we get

$$
\left\|Q_{t, s}\right\|_{L_{1} \rightarrow B_{b}} \leq c \widetilde{G}_{s-t}(0)(s-t)^{-1+\varepsilon_{0}}, \quad 0<s-t \leq \tau .
$$

We have that $\left\|P_{t, s}^{(0)}\right\|_{B_{b} \rightarrow B_{b}}$ is bounded and, in addition, by (23)

$$
\left\|P_{t, s}^{(0)}\right\|_{L_{1} \rightarrow L_{\infty}} \leq c G_{s-t}(0)
$$

Then using the parametrix representation (27) and repeating the argument above we get

$$
\left\|P_{t, s}-P_{t, s}^{(0)}\right\|_{L_{1} \rightarrow L_{\infty}} \leq c G_{s-t}(0)(s-t)^{\varepsilon_{0}}, \quad 0<s-t \leq \tau .
$$


This is actually a uniform-in- $x, y$ bound for the residual term $r_{t, s}(x, y)$ in the decomposition obtained by the parametrix method. To complete the proof of Theorem 2.2, we prove the corresponding analogue of (37), see (116):

$$
\left\|\widetilde{P}_{t, s}-P_{t, s}^{(0)}\right\|_{L_{1} \rightarrow L_{\infty}} \leq c G_{s-t}(0)(s-t)^{\varepsilon_{0}}, \quad 0<s-t \leq \tau .
$$

The proof of Theorem 2.3 (postponed to Section 5) stating the Hölder continuity for the evolutionary family $\left\{P_{t, s}\right\}$, is based on the parametrix representation (24) for this family and the Hölder continuity of the family $\left\{P_{t, s}^{(0)}\right\}$ involved in this representation.

\section{One-Dimensional DENSITY}

This section is devoted to the study of one-dimensional components of the process $Z=\left(Z_{1}, \ldots, Z_{d}\right)$. Recall that the characteristic exponent of $Z_{i}$ is $\psi_{i}$. In this section we fix $i \in\{1, \ldots, d\}$ and $\psi$ denotes the fixed $\psi_{i}$. By $\nu, h$ and $\tilde{g}$ we denote the corresponding Lévy measure, the Pruitt function and the transition density, respectively. We will construct a truncated version $g$ of the transition density $\tilde{g}$. We will show various estimates of $g, \tilde{g}$ and its derivatives. These construction and estimates will play a crucial role to make the parametrix construction in Section 5 work.

For $r>0$ we put

$$
K(r)=\int_{\{x \in \mathbb{R}:|x| \leq r\}}|x|^{2} r^{-2} \nu(d x) .
$$

We have the following relationship between $K(r)$ and $h(r)$ [16, Lemma 2.2],

$$
h(r)=2 \int_{r}^{\infty} K(w) w^{-1} d w, r>0 .
$$

We observe that, due to infinitness of the Lévy measure $K(w)>0, w>0$, hence $h(r)$ is strictly decreasing on $(0, \infty)$.

Clearly, $r^{2} h(r), r^{2} K(r)$ are increasing on $(0, \infty)$. Using the monotonicity of the function $r^{2} h(r)$ we can easily extend (6) to all $\theta>0$,

$$
C_{1} \lambda^{-\alpha} h(\theta)\left(1 \vee \theta^{2}\right)^{-1} \leq h(\lambda \theta) \leq C_{2} \lambda^{-\beta} h(\theta)\left(1 \vee \theta^{2}\right), \quad 0<\lambda \leq 1 .
$$

Let us observe that the scaling property (6) is equivalent to

$$
C_{1}^{1 / \alpha} h^{-1}(\theta) \lambda^{-1 / \alpha} \leq h^{-1}(\lambda \theta) \leq C_{2}^{1 / \beta} h^{-1}(\theta) \lambda^{-1 / \beta}, \theta>h(1), \lambda \geq 1 .
$$

Morever this can be extended to all $\theta>0$ (via (39)),

$$
C_{1}^{1 / \alpha} h^{-1}(\theta) \lambda^{-1 / \alpha}\left(1 \vee h^{-1}(\theta)^{2}\right)^{-1} \leq h^{-1}(\lambda \theta) \leq C_{2}^{1 / \beta} h^{-1}(\theta) \lambda^{-1 / \beta}\left(1 \vee h^{-1}(\theta)^{2}\right), \quad \lambda \geq 1
$$

The following important result was essentially proved in [16, Lemma 2.3]. For the reader convenience we provide its proof.

Lemma 4.1. Let $c=\left(\frac{2}{C_{1}}\right)^{2 / \alpha}-1$. For $0<r \leq r_{0}$ we have

$$
h(r) \leq c\left(1 \vee r_{0}^{2}\right) K(r) .
$$

Moreover, for $|\xi| \geq \xi_{0}>0$,

$$
\frac{1}{4 c\left(1 \vee \xi_{0}^{-2}\right)} h(1 /|\xi|) \leq \psi(\xi) \leq 2 h(1 /|\xi|)
$$

Proof. Let $r \leq 1$ and $\lambda_{0}=\left(\frac{C_{1}}{2}\right)^{1 / \alpha}<1$. Then, by (6), we have

$$
2 h(r) \leq h\left(\lambda_{0} r\right) \text {. }
$$


Next, by (38) and monotonicity of $w^{2} K(w)$ we obtain

$$
h(r) \leq h\left(\lambda_{0} r\right)-h(r)=2 \int_{\lambda_{0} r}^{r} w^{2} K(w) w^{-3} d w \leq 2 r^{2} K(r) \int_{\lambda_{0} r}^{r} w^{-3} d w
$$

Hence

$$
h(r) \leq \frac{1-\lambda_{0}^{2}}{\lambda_{0}^{2}} K(r)=\left(\left(\frac{2}{C_{1}}\right)^{2 / \alpha}-1\right) K(r), r \leq 1 .
$$

If $r_{0}>1$, then for $1 \leq r \leq r_{0}$ then, by monotonicity of $w^{2} K(w)$ and $w^{2} h(w)$, we have

$$
r^{2} h(r) \leq r_{0}^{2} h\left(r_{0}\right) \frac{r^{2} K(r)}{K(1)} .
$$

Hence,

$$
h(r) \leq r_{0}^{2} \frac{h(1)}{K(1)} K(r) \leq\left(\left(\frac{2}{C_{1}}\right)^{2 / \alpha}-1\right) r_{0}^{2} K(r) .
$$

The proof of (42) is completed.

Next, by the inequality $1-\cos x \geq x^{2} / 4$ for $|x| \leq 1$, we obtain

$$
(1 / 4) K(1 /|\xi|) \leq \psi(\xi)=\int_{\mathbb{R}}(1-\cos (\xi x)) d \nu(x) \leq 2 h(1 /|\xi|) .
$$

Applying (42) we have

$$
\psi(\xi) \geq \frac{1}{4 c\left(1 \vee \xi_{0}^{-2}\right)} h(1 /|\xi|),|\xi| \geq \xi_{0},
$$

which completes the proof.

Now, we can give the arguments that (6) is equivalent to (7). If (6) holds, then (43) with $\xi_{0}=1$ implies (7) with $C_{1}^{*}=\frac{C_{1}}{8 c}, C_{2}^{*}=8 c C_{2}$, where $c=\left(2 / C_{1}\right)^{2 / \alpha}-1$.

On the other hand (7) implies the same scaling conditions for the maximal function $\psi^{*}(\xi)=\sup _{|x| \leq|\xi|} \psi^{*}(\xi)$. Then (66) holds, since due to [14, Lemma 4] we have $\psi^{*}(\xi) \asymp$ $h(1 /|\xi|), \xi \in \mathbb{R}$.

Lemma 4.2. Let $\tau>0$. For $0<u \leq \tau$ we have

$$
c_{1} u^{1 / \alpha} \leq h^{-1}(1 / u) \leq c_{2} u^{1 / \beta},
$$

where $c_{1}=C_{1}^{1 / \alpha}\left(h(1) \wedge \frac{1}{\tau}\right)^{1 / \alpha}$ and $c_{2}=C_{2}^{1 / \beta}\left(h^{-1}\left(\frac{1}{\tau}\right) \vee 1\right) h(1)^{1 / \beta}$.

Proof. Taking $\theta=1$ we can rewrite (6) as

$$
C_{1}^{1 / \alpha} h(1)^{1 / \alpha} h(\lambda)^{-1 / \alpha} \leq \lambda \leq C_{2}^{1 / \beta} h(1)^{1 / \beta} h(\lambda)^{-1 / \beta}, 0<\lambda \leq 1 .
$$

Putting $\lambda=h^{-1}(s)$, for $s \geq h(1)$, we have $\left(C_{1} h(1)\right)^{1 / \alpha} s^{-1 / \alpha} \leq h^{-1}(s) \leq\left(C_{2} h(1)\right)^{1 / \beta} s^{-1 / \beta}$.

If $0<s_{0} \leq s \leq h(1)$ we have $s_{0}^{1 / \alpha} s^{-1 / \alpha} \leq h^{-1}(s) \leq h^{-1}\left(s_{0}\right) h(1)^{1 / \beta} s^{-1 / \beta}$. Choosing $\frac{1}{s}=u \leq \tau$ we show that

$$
C_{1}^{1 / \alpha}\left(\frac{1}{\tau} \wedge h(1)\right)^{1 / \alpha} t^{1 / \alpha} \leq h^{-1}(1 / t) \leq C_{2}^{1 / \beta} h^{-1}\left(\frac{1}{\tau} \wedge h(1)\right) h(1)^{1 / \beta} t^{1 / \beta} .
$$

Now we state an easy corollary to (40) and Lemma 4.2 
Corollary 4.3. Let $0<\varepsilon<1$ and $0<u \leq \tau<\infty$. We have

$$
\frac{h^{-1}\left(u^{\varepsilon-1}\right)}{h^{-1}\left(u^{-1}\right)} \leq c u^{-\varepsilon / \alpha}
$$

where $c=c\left(h(1), h^{-1}(1 / \tau), h^{-1}(1), \tau, \alpha, \varepsilon, C_{1}\right)$.

If $\tau_{0}=(h(1) \vee 1)^{-\frac{1}{1-\varepsilon}}$, then for $u \leq \tau_{0}$,

$$
\frac{h^{-1}\left(u^{\varepsilon-1}\right)}{h^{-1}\left(u^{-1}\right)} \leq C_{1}^{-1 / \alpha} u^{-\varepsilon / \alpha} .
$$

Moreover, for $0<\lambda \leq 1$,

$$
\frac{h^{-1}(1 / u)}{h^{-1}(1 /(\lambda u))} \leq C_{1}^{-1 / \alpha} \lambda^{-1 / \alpha}\left(h^{-1}\left(\tau^{-1}\right) \vee 1\right)^{2} .
$$

Proof. Let $u \leq \tau_{0}=(h(1) \vee 1)^{-1 /(1-\varepsilon)}$. We apply (40) with $\lambda=u^{-\varepsilon}$ and $\theta=u^{-1+\varepsilon}$ to get

$$
\frac{h^{-1}\left(u^{\varepsilon-1}\right)}{h^{-1}\left(u^{-1}\right)}=\frac{h^{-1}\left(u^{\varepsilon-1}\right)}{h^{-1}\left(u^{-\varepsilon} u^{\varepsilon-1}\right)} \leq C_{1}^{-1 / \alpha} u^{-\varepsilon / \alpha} .
$$

If $\tau_{0} \leq u \leq \tau$ then by monotonicity of $h^{-1}$,

$$
h^{-1}\left(u^{\varepsilon-1}\right) \leq h^{-1}(1 / \tau) \vee h^{-1}(1) \text { and } h^{-1}\left(u^{-1}\right) \geq h^{-1}\left(\tau_{0}^{-1}\right) .
$$

Moreover, by Lemma 4.2, we obtain

$$
h^{-1}\left(\tau_{0}^{-1}\right) \geq C_{1}^{1 / \alpha}\left(\frac{1}{\tau} \wedge h(1)\right)^{1 / \alpha} \tau_{0}^{1 / \alpha} .
$$

It follows that for $\tau_{0} \leq u \leq \tau$,

$$
\frac{h^{-1}\left(u^{\varepsilon-1}\right)}{h^{-1}\left(u^{-1}\right)} \leq \frac{h^{-1}(1 / \tau) \vee h^{-1}(1)}{C_{1}^{1 / \alpha}\left(\frac{1}{\tau} \wedge h(1)\right)^{1 / \alpha}} \tau_{0}^{1 / \alpha} \tau^{\varepsilon / \alpha} u^{-\varepsilon / \alpha} .
$$

Now we start to construct a truncated version $g$ of the transtion density $\tilde{g}$. Fix $\varepsilon \in$ $\left(0, \varepsilon_{0}\right]$, where $\varepsilon_{0}<1$ is defined in Remark [5.1. Let for $u>0$

$$
R_{u}=R_{u}(\varepsilon)=h^{-1}\left(\frac{1}{u^{1-\varepsilon}}\right)
$$

and

We have

$$
\psi_{u}(\xi)=\int_{|v| \leq R_{u}}(1-\cos (v \xi)) \nu(d v)
$$

To prove (48) we note that

$$
\psi_{u}(\xi) \geq \psi(\xi)-2 u^{\varepsilon-1} .
$$

$$
\int_{|v|>R_{u}}(1-\cos (v \xi)) \nu(d v) \leq 2 \int_{|v|>R_{u}} \nu(d v) \leq 2 h\left(R_{u}\right)=2 u^{\varepsilon-1},
$$

hence

$$
\psi_{u}(\xi)=\psi(\xi)-\int_{|v|>R_{u}}(1-\cos (v \xi)) \nu(d v) \geq \psi(\xi)-2 u^{\varepsilon-1} .
$$

Let $0<u<\infty$ and $w \in \mathbb{R}$. Put

$$
g_{u}(w)=\frac{1}{2 \pi} \int_{\mathbb{R}} e^{i w z} e^{-\int_{0}^{u} \psi_{r}(z) d r} d z .
$$

Since, by (48) and then by (77), $\int_{0}^{u} \psi_{r}(z) d r \geq u \psi(z)-(2 / \varepsilon) u^{\varepsilon} \geq c|z|^{\alpha}$ for $|z|$ large enough, the function $g_{u}$ is well defined density function such that $g_{u} \in C^{\infty}(\mathbb{R})$. 
For $0<u<\infty$ and a measurable set $D \subset \mathbb{R}$ put

$$
\nu_{u}(D)=\int_{0}^{u} \int_{|x| \leq R_{r}} 1_{D}(x) \nu(d x) d r .
$$

It is clear that $\nu_{u}$ is the Lévy measure of the infinitely divisible density $g_{u}$.

For any $u>0$ put

$$
m_{u}=\int_{\mathbb{R}} x^{2} \nu_{u}(d x) .
$$

Lemma 4.4. For any $\tau>0$ there is a constant $c=c\left(\alpha, C_{1}, h^{-1}(1 / \tau), h^{-1}(1)\right)$ such that for $u \leq \tau$ we have

$$
c R_{u}^{2} u^{\varepsilon} \leq m_{u} \leq R_{u}^{2} u^{\varepsilon} .
$$

The upper bound holds for any $u>0$.

$$
\begin{aligned}
& \text { If } u \leq \tau_{0}=h(1)^{-\frac{1}{1-\varepsilon}} \text {, then for } u \leq \tau_{0} \\
& \qquad m_{u} \geq c R_{u}^{2} u^{\varepsilon}
\end{aligned}
$$

with $c=c\left(C_{1}, \alpha\right)$.

Proof. The upper bound is clear:

$$
m_{u}=\int_{\mathbb{R}} x^{2} \nu_{u}(d x) \leq u \int_{|x| \leq R_{u}} x^{2} \nu(d x) \leq u R_{u}^{2} h\left(R_{u}\right)=R_{u}^{2} u^{\varepsilon},
$$

The lower bound follows from the scaling property. By (47),

$$
\frac{R_{u}}{R_{u / 2}} \leq\left(\frac{2^{1-\varepsilon}}{C_{1}}\right)^{1 / \alpha}\left(1 \vee h^{-1}\left(\frac{1}{\tau^{1-\varepsilon}}\right)\right)^{2} .
$$

By Lemma 4.1, we have for $r \leq R_{\tau}$,

$$
\frac{h(r)}{K(r)} \leq\left(\frac{2}{C_{1}}\right)^{2 / \alpha}\left(1 \vee h^{-1}\left(\frac{1}{\tau^{1-\varepsilon}}\right)\right)^{2} .
$$

Observing that $\left.h^{-1}\left(\frac{1}{\tau^{1-\varepsilon}}\right) \leq h^{-1}(1) \vee h^{-1}(1 / \tau)\right)$ and taking $c=\left(\frac{2}{C_{1}}\right)^{-2 / \alpha}\left(1 \vee h^{-1}(1) \vee h^{-1}(1 / \tau)\right)^{-2}$ we have $\operatorname{ch}(r) \leq K(r)$ for $r \leq R_{\tau}$. Moreover $c R_{u} \leq R_{u / 2}$ for $u \leq \tau$. Then using monotonicity of $h$ we have

$$
\begin{aligned}
m_{u} & =\int_{0}^{u} \int_{|x| \leq R_{r}} x^{2} \nu(d x) d r=\int_{0}^{u} R_{r}^{2} K\left(R_{r}\right) d r \\
& \geq c \int_{u / 2}^{u} R_{r}^{2} h\left(R_{r}\right) d r \geq \frac{c}{2} u R_{u / 2}^{2} h\left(R_{u}\right) \geq \frac{c^{2}}{2} u R_{u}^{2} h\left(R_{u}\right)=\frac{c^{2}}{2} R_{u}^{2} u^{\varepsilon} .
\end{aligned}
$$

Lemma 4.5. Let $\gamma>\beta$. For any $\tau>0$ there is a constant $c=c\left(C_{2}, \beta, \gamma, h^{-1}(1 / \tau), h^{-1}(1)\right)$ such that for $u \leq \tau$ we have

$$
\int_{|x| \leq R_{u}}|x|^{\gamma} \nu(d x) \leq c R_{u}^{\gamma} h\left(R_{u}\right)=c R_{u}^{\gamma} u^{-1+\varepsilon} .
$$

Moreover for $u \leq \tau_{0}=h(1)^{-\frac{1}{1-\varepsilon}}$ the above constant $c=c\left(C_{2}, \beta, \gamma\right)$.

If $\gamma=2$ the above inequality holds for any $u>0$ and with $c=1$.

Proof. Let $L(r)=\nu([r, \infty)), r>0$. By integration by parts

$$
\int_{0<x \leq R_{u}} x^{\gamma} \nu(d x) \leq \limsup _{r \rightarrow 0^{+}} r^{\gamma} L(r)+\gamma \int_{0<x \leq R_{u}} x^{\gamma-1} L(x) d x .
$$


Next, by (6),

which implies

$$
\limsup _{r \rightarrow 0^{+}} r^{\gamma} L(r) \leq \limsup _{r \rightarrow 0^{+}} r^{\gamma} h(r)=0
$$

$$
\int_{0<x \leq R_{u}} x^{\gamma} \nu(d x) \leq \gamma \int_{0<x \leq R_{u}} x^{\gamma-1} L(x) d x \leq \gamma \int_{0<x \leq R_{u}} x^{\gamma-1} h(x) d x .
$$

It follows from (6) that, if $R_{u} \leq 1$, then

$$
h(x)=h\left(R_{u}\left(x / R_{u}\right)\right) \leq C_{2}\left(x / R_{u}\right)^{-\beta} h\left(R_{u}\right), x \leq R_{u} .
$$

If $1 \leq R_{u} \leq R_{\tau}$, since $r^{2} h(r)$ is an increasing function and by (6), we have

$$
h(x) \leq h\left(x / R_{u}\right) \leq C_{2}\left(x / R_{u}\right)^{-\beta} h\left(R_{u}\right) R_{u}^{2}, x \leq R_{u} .
$$

The last two estimates yield

$\int_{0<x \leq R_{u}} x^{\gamma-1} h(x) d x \leq C_{2}\left(R_{\tau}^{2} \vee 1\right) R_{u}^{\beta} h\left(R_{u}\right) \int_{0<x \leq R_{u}} x^{\gamma-\beta-1} d x=C_{2}\left(R_{\tau}^{2} \vee 1\right) \frac{1}{\gamma-\beta} R_{u}^{\gamma} h\left(R_{u}\right)$.

This together with the estimate

$$
R_{\tau}=h^{-1}\left(\frac{1}{\tau^{1-\varepsilon}}\right) \leq h^{-1}(1 / \tau) \vee h^{-1}(1)
$$

end the proof for arbitrary $\gamma>\beta$. Moreover, for $\tau=\tau_{0}$, we have $R_{\tau}=1$ which shows that the constant $c=C_{2} \frac{\gamma}{\gamma-\beta}$.

The assertion of the lemma for $\gamma=2$ is a consequence of the definition of the function $h$.

Lemma 4.6. Let $\gamma \geq 0$ and $\tau>0$. There are constants $c_{1}=c_{1}\left(\alpha, h^{-1}(1 / \tau), \tau, \gamma, \varepsilon, C_{1}\right)$ and $c_{2}=c_{2}(\gamma)$ such that for any $0<u \leq \tau$,

$$
\frac{c_{2}}{h^{-1}(1 / u)^{\gamma+1}} \leq \int_{\mathbb{R}}|z|^{\gamma} e^{-\int_{0}^{u} \psi_{r}(z) d r} d z \leq \frac{c_{1}}{h^{-1}(1 / u)^{\gamma+1}} .
$$

If $\tau=1 / h(1)$ then the constant $c_{1}=c_{1}\left(h(1), \varepsilon, \alpha, \gamma, C_{1}\right)$.

Proof. By (48),

Next,

$$
\int_{0}^{u} \psi_{r}(z) d r \geq u \psi(z)-(2 / \varepsilon) u^{\epsilon}
$$

Moreover, by Lemma 4.1,

$$
\int_{\mathbb{R}}|z|^{\gamma} e^{-\int_{0}^{u} \psi_{r}(z) d r} d z \leq e^{(2 / \varepsilon) u^{\varepsilon}} \int_{\mathbb{R}}|z|^{\gamma} e^{-u \psi(z)} d z .
$$

$$
\int_{\mathbb{R}}|z|^{\gamma} e^{-u \psi(z)} d z \leq 2+\int_{|z|>1}|z|^{\gamma} e^{-c u h(1 /|z|)} d z,
$$

where $c=\frac{1}{4\left(\left(\frac{2}{C_{1}}\right)^{2 / \alpha}-1\right)}$. By the same arguments as in the proof of [1, Lemma 16] we get

$$
\int_{\mathbb{R}}|z|^{\gamma} e^{-u c h(1 /|z|)} d z \leq \frac{c_{*}}{h^{-1}(1 / u)^{\gamma+1}}, u \leq 1 / h(1),
$$

where $c_{*}=c_{*}\left(\gamma, \alpha, C_{1}\right)$. Since $h^{-1}(1 / u) \leq 1$ for $u \leq 1 / h(1)$, it follows that

$$
\int_{\mathbb{R}}|z|^{\gamma} e^{-u \psi(z)} d z \leq \frac{c^{*}}{h^{-1}(1 / u)^{\gamma+1}}, u \leq 1 / h(1),
$$

where $c^{*}=c_{*}+2$. If $1 / h(1) \leq u \leq \tau$, then from the above estimate and monotonicity of $h^{-1}$ we obtain

$$
\int_{\mathbb{R}}|z|^{\gamma} e^{-u \psi(z)} d z \leq \frac{c^{*} h^{-1}(1 / \tau)^{\gamma+1}}{h^{-1}(1 / u)^{\gamma+1}} .
$$


Finally we conclude that

$$
\int_{\mathbb{R}}|z|^{\gamma} e^{-u \psi(z)} d z \leq c^{*}\left(h^{-1}(1 / \tau) \vee 1\right)^{\gamma+1} \frac{1}{h^{-1}(1 / u)^{\gamma+1}}
$$

for $u \leq \tau$. The proof of the upper bound is completed.

To get the lower bound we observe that

$$
\int_{0}^{u} \psi_{r}(z) d r \leq u \psi(z) \leq 2 u h(1 /|z|)
$$

Hence, denoting $a=\frac{1}{h^{-1}(1 / u)}$ we arrive at

$$
\int_{\mathbb{R}}|z|^{\gamma} e^{-\int_{0}^{u} \psi_{r}(z) d r} d z \geq \int_{-a}^{a}|z|^{\gamma} e^{-u 2 h(1 /|z|)} d z \geq 2 e^{-2} \int_{0}^{a}|z|^{\gamma} d z=2 e^{-2} \frac{1}{\gamma+1} a^{\gamma+1}
$$

which ends the proof of the lower bound.

Corollary 4.7. For $0<u \leq \tau$

$$
\frac{c_{2}}{2 \pi h^{-1}(1 / u)} \leq g_{u}(0) \leq \frac{c_{1}}{2 \pi h^{-1}(1 / u)}
$$

where $c_{1}, c_{2}$ are constants from (49) corresponding to $\gamma=0$.

The function $(0, \infty) \times \mathbb{R} \ni(u, x) \rightarrow g_{u}(x)$ is continuous.

Proof. Since

$$
g_{u}(x)=\frac{1}{2 \pi} \int_{\mathbb{R}} e^{i x z} e^{-\int_{0}^{u} \psi_{r}(z) d r} d z .
$$

we get, by Lemma 4.6, the lower and upper estimate of $g_{u}(0)$. The continuity follows from the continuity of the map $(0, \infty) \times \mathbb{R} \ni(u, x) \rightarrow e^{i x z} e^{-\int_{0}^{u} \psi_{r}(z) d r}$, the upper estimate in (49) and the bounded convergence theorem.

Lemma 4.8. For any $u>0$ we have

$$
\begin{gathered}
\sup _{x \in \mathbb{R}}\left|g_{u}(x)-\tilde{g}_{u}(x)\right| \leq 2 \frac{u^{\varepsilon}}{\varepsilon} g_{u}(0), \\
\int_{\mathbb{R}}\left|g_{u}(x)-\tilde{g}_{u}(x)\right| d x \leq 2 \frac{u^{\varepsilon}}{\varepsilon}
\end{gathered}
$$

and

$$
\tilde{g}_{u}(0) \leq g_{u}(0) \leq \tilde{g}_{u}(0) e^{\frac{u^{\varepsilon}}{\varepsilon}}
$$

Proof. The proof is similar to the proof of Proposition C.9 in [22]. Let $u \in(0, \tau]$ and $x \in \mathbb{R}$ be arbitrary. For any $z \in \mathbb{R}$ we have

$$
u \psi(z)=\int_{0}^{u} \psi_{r}(z) d r+\int_{0}^{u} \int_{|v|>R_{r}}(1-\cos (v z)) \nu(d v) d r
$$

It follows that

$$
\begin{aligned}
\tilde{g}_{u}(x) & =\frac{1}{2 \pi} \int_{\mathbb{R}} e^{i x z} e^{-u \psi(z)} d z \\
& =\frac{1}{2 \pi} \int_{\mathbb{R}} e^{i x z} e^{-\int_{0}^{u} \psi_{r}(z) d r} e^{-\int_{0}^{u} \int_{|v|>R_{r}}(1-\cos (v z)) \nu(d v) d r} d z \\
& =\int_{\mathbb{R}} g_{u}(x-z) P_{u}^{\text {tail }}(d z),
\end{aligned}
$$

where $P_{u}^{\text {tail }}(d z)$ is the exponential (for the convolution) of the measure $\Lambda_{u}^{\text {tail }}$ i.e.

$$
P_{u}^{\text {tail }}(A)=e^{-\Lambda_{u}^{\text {tail }}(\mathbb{R})} \sum_{k=0}^{\infty} \frac{1}{k !}\left(\Lambda_{u}^{\text {tail }}\right)^{* k}(A), \quad A \in \mathcal{B}(\mathbb{R}),
$$


where $\Lambda_{u}^{\text {tail }}(A)=\int_{0}^{u} \nu\left(\left\{v \in A:|v|>R_{r}\right\}\right) d r$. We have

$$
\Lambda_{u}^{\text {tail }}(\mathbb{R})=\int_{0}^{u} \nu\left(\left\{v \in \mathbb{R}:|v|>R_{r}\right\}\right) d r \leq \int_{0}^{u} h\left(R_{r}\right) d r=\frac{u^{\varepsilon}}{\varepsilon} .
$$

It follows that

$$
\left|1-e^{-\Lambda_{u}^{\text {tail }}(\mathbb{R})}\right| \leq \frac{u^{\varepsilon}}{\varepsilon} .
$$

Moreover, by (54) and (55), we get

$$
\begin{aligned}
\left|\tilde{g}_{u}(x)-g_{u}(x)\right| & \leq g_{u}(x)\left|1-e^{-\Lambda_{u}^{\text {tail }}(\mathbb{R})}\right| \\
& +e^{-\Lambda_{u}^{\text {tail }}(\mathbb{R})} \sum_{k=1}^{\infty} \int_{\mathbb{R}} \frac{1}{k !} g_{u}(x-z)\left(\Lambda_{u}^{\text {tail }}\right)^{* k}(d z) .
\end{aligned}
$$

Using this, (56) and (57) we get (51). Integrating (58) and using (56), (57) we get (52).

Applying (54) with $x=0$ we obtain $g_{u}(0) e^{-\Lambda_{u}^{\text {tail }}(\mathbb{R})} \leq \tilde{g}_{u}(0) \leq g_{u}(0)$, which combined with (56) proves (53).

Let for $u>0, \xi, w \in \mathbb{R}$

$$
v_{u}(\xi, w)=-\xi w+\int_{\mathbb{R}}(\cosh (\xi v)-1) \nu_{u}(d v) .
$$

Lemma 4.9. Fix $u>0, w \in \mathbb{R}$. Let $\xi_{0} \in \mathbb{R}$ be such that

$$
v_{u}\left(\xi_{0}, w\right)=\inf _{\xi \in \mathbb{R}} v_{u}(\xi, w) .
$$

Then,

$$
\left|\xi_{0}\right| \leq 2 \frac{|w|}{m_{u}}
$$

Proof. We have

$$
\int_{\mathbb{R}}(\cosh (\xi z)-1) \nu_{u}(d z) \geq \frac{1}{2} \int_{\mathbb{R}} \xi^{2} z^{2} \nu_{u}(d z)=\frac{1}{2} \xi^{2} m_{u}
$$

Hence $v_{u}(\xi, w) \geq-\xi w+\xi^{2} m_{u} / 2$. Since $v_{u}\left(\xi_{0}, w\right) \leq v_{u}(0, w)=0$ we have $-\left|\xi_{0}\right||w|+$ $\xi_{0}^{2} m_{u} / 2 \leq 0$, which gives (59).

Lemma 4.10. Let $\tau>0$. For any $0<u \leq \tau, w \in \mathbb{R}, k \in \mathbb{N}_{0}$ we have

$$
\left|\frac{d^{k}}{d w^{k}} g_{u}(w)\right| \leq c_{k}\left(\frac{1}{h^{-1}(1 / u)}\right)^{k} g_{u}(0)
$$

and

$$
\left|\frac{d^{k}}{d w^{k}} g_{u}(w)\right| \leq c_{k}\left(\frac{1}{u^{\varepsilon} h^{-1}(1 / u)}\right)^{k} e^{-\frac{|w|}{8 R_{u}}} g_{u}(0) .
$$

The constant $c_{k}$ depends on $C_{1}, \alpha, \varepsilon, \tau, h^{-1}(1 / \tau), h^{-1}(1)$ and $k$. If $\tau=\tau_{0}=(h(1) \vee 1)^{-\frac{1}{1-\varepsilon}}$, then the constant $c_{k}$ depends on $k, C_{1}, \alpha, \varepsilon, h(1)$.

Proof. The proof of (60) follows immediately from Lemma 4.6 and Corollary 4.7. Let

We have

$$
Q_{u}(\xi, w)=i \xi w-\int_{0}^{u} \psi_{r}(\xi) d r, u>0, \xi, w \in \mathbb{R}
$$

$$
g_{u}(w)=\frac{1}{2 \pi} \int_{\mathbb{R}} e^{Q_{u}(\xi, w)} d \xi
$$


For any $k \in \mathbb{N}_{0}$ we get

$$
\frac{d^{k}}{d w^{k}} g_{u}(w)=\frac{1}{2 \pi} \int_{\mathbb{R}} i^{k} \xi^{k} e^{Q_{u}(\xi, w)} d \xi
$$

Recall that $\xi_{0}=\arg \min _{\xi} v_{u}(\xi, w)$. We proceed in a similar way as in [23] where a bound on the transition density was derived. Note that functions $\psi_{r}$ and $Q_{u}(\cdot, w)$ can be extended analytically to $\mathbb{C}$. Applying the Cauchy-Poincare theorem (justification is exactly the same as in the proof of Theorem 6 of [23]) we claim that

$$
\left|\frac{d^{k}}{d w^{k}} g_{u}(w)\right|=\frac{1}{2 \pi}\left|\int_{\mathbb{R}}\left(\xi+i \xi_{0}\right)^{k} e^{Q_{u}\left(\xi+i \xi_{0}, w\right)} d \xi\right| \text {. }
$$

Observe that

$$
\operatorname{Re} Q_{u}\left(\xi+i \xi_{0}, w\right) \leq v_{u}\left(\xi_{0}, w\right)-\int_{0}^{u} \psi_{r}(\xi) d r
$$

Hence

$$
\begin{aligned}
\left|\frac{d^{k}}{d w^{k}} g_{u}(w)\right| & \leq \int_{\mathbb{R}}\left(|\xi|+\left|\xi_{0}\right|\right)^{k} e^{v_{u}\left(\xi_{0}, w\right)-\int_{0}^{u} \psi_{r}(\xi) d r} d \xi \\
& \leq 2^{k} e^{v_{u}\left(\xi_{0}, w\right)} \int_{\mathbb{R}}\left(|\xi|^{k}+\left|\xi_{0}\right|^{k}\right) e^{-\int_{0}^{u} \psi_{r}(\xi) d r} d \xi
\end{aligned}
$$

Now, we will show that for any $w \in \mathbb{R}$ we have

$$
\begin{aligned}
v_{u}\left(\xi_{0}, w\right) & \leq \frac{e m_{u}}{2 R_{u}^{2}}-\frac{|w|}{4 R_{u}} \\
& \leq \frac{e}{2} u^{\epsilon}-\frac{|w|}{4 R_{u}} .
\end{aligned}
$$

If $|w| \leq \frac{2 e m_{u}}{R_{u}}$, then

$$
\frac{e m_{u}}{2 R_{u}^{2}}-\frac{|w|}{4 R_{u}} \geq 0=v_{u}(0, w) \geq v_{u}\left(\xi_{0}, w\right),
$$

which proves $(62)$ in this case.

If $|w| \geq \frac{2 e m_{u}}{R_{u}}$, to prove (62), we use the arguments as in [35, proof of Lemma 4.2], so we omit the details.

Next, observe that (63) follows from Lemma 4.4.

We also have, by (49),

$$
\int_{\mathbb{R}}|\xi|^{k} e^{-\int_{0}^{u} \psi_{r}(\xi) d r} d \xi \leq \frac{c_{k}}{h^{-1}(1 / u)^{k+1}}
$$

By Lemma 4.9

$$
\int_{\mathbb{R}}\left|\xi_{0}\right|^{k} e^{-\int_{0}^{u} \psi_{r}(\xi) d r} d \xi \leq c_{k}|w|^{k} \frac{c}{m_{u}^{k} h^{-1}(1 / u)}
$$

Hence

$$
\begin{aligned}
\left|\frac{d^{k}}{d w^{k}} g_{u}(w)\right| & \leq \frac{c_{k}}{h^{-1}(1 / u)^{k+1}}\left(1+\frac{R_{u} h^{-1}(1 / u)}{m_{u}} \frac{|w|}{R_{u}}\right)^{k} e^{-\frac{|w|}{4 R_{u}}} \\
& \leq \frac{c_{k}}{h^{-1}(1 / u)^{k+1}}\left(1+\frac{R_{u} h^{-1}(1 / u)}{m_{u}}\right)^{k} e^{-\frac{|w|}{8 R_{u}}}
\end{aligned}
$$

Next, we observe that, by Lemma 4.4 and since $h^{-1}(1 / u) \leq\left(\frac{h^{-1}(1 / \tau)}{h^{-1}(1)} \vee 1\right) R_{u}$, we obtain

which implies

$$
\frac{R_{u} h^{-1}(1 / u)}{m_{u}} \leq c \frac{h^{-1}(1 / u)}{u^{\varepsilon} R_{u}} \leq \frac{c}{u^{\varepsilon}}
$$




$$
\left|\frac{d^{k}}{d w^{k}} g_{u}(w)\right| \leq \frac{c_{k}}{u^{k \varepsilon} h^{-1}(1 / u)^{k+1}} e^{-\frac{|w|}{8 R_{u}}} .
$$

Finally, we note that all the constants in the case $\tau=\tau_{0}=(h(1) \vee 1)^{-\frac{1}{1-\varepsilon}}$ are dependent only on $k, C_{1}, \alpha, \varepsilon, h(1)$. It follows from the appropriate parts of Lemma 4.4 and Lemma 4.6.

Let for $0<u<\infty$ and $f \in C^{2}(\mathbb{R})$

$$
K_{u} f(w)=\mathrm{P} . \mathrm{V} \cdot \int_{|z| \leq R_{u}}(f(w+z)-f(w)) \nu(d z), w \in \mathbb{R} .
$$

For $f \in L^{1}(\mathbb{R})$ and $\xi \in \mathbb{R}$ denote $\hat{f}(\xi)=\int_{\mathbb{R}} e^{-i \xi x} f(x) d x$. If $f \in C^{2}(\mathbb{R}) \cap L^{1}(\mathbb{R})$ is such that $\lim \sup _{|x| \rightarrow \infty}|x|^{1+\delta}\left|f^{\prime \prime}(x)\right|<\infty$ for some $\delta>0$ we observe that $K_{u} f \in L^{1}(\mathbb{R})$ and

$$
\widehat{K_{u} f}(\xi)=-\psi_{u}(\xi) \hat{f}(\xi), \xi \in \mathbb{R} .
$$

Next, by (61), the above requirements are satisfied for $f=g_{u}$.

We have $\hat{g}_{u}(\xi)=e^{-\int_{0}^{u} \psi_{r}(\xi) d r}$, so for any $0<u<\infty$ and $\xi \in \mathbb{R}$

$$
\frac{\partial}{\partial u} \hat{g}_{u}(\xi)+\psi_{u}(\xi) \hat{g}_{u}(\xi)=0 .
$$

By (64), it follows that for any $0<u<\infty$ and $w \in \mathbb{R}$ we have

$$
\frac{\partial}{\partial u} \hat{g}_{u}(w)-\widehat{K_{u} g_{u}}(w)=0 .
$$

Next we claim that

$$
\frac{\partial}{\partial u} g_{u}(w)=\frac{1}{2 \pi} \int_{\mathbb{R}} e^{i w z} \frac{\partial}{\partial u} \hat{g_{u}}(z) d z .
$$

This follows from the estimate

$$
\left|\frac{\partial}{\partial u} \hat{g_{u}}(z)\right|=\psi_{u}(z) \hat{g}_{u}(z) \leq e^{(2 / \varepsilon) u^{\varepsilon}} \psi(z) e^{-u \psi(z)},
$$

which is implied by (48). Next we use (65) to get

$$
\frac{\partial}{\partial u} g_{u}(w)=\frac{1}{2 \pi} \int_{\mathbb{R}} e^{i w z} \widehat{K_{u} g_{u}}(z) d z=K_{u} g_{u}(w),
$$

where we have equality almost surely. By continuity we have it everywhere.

\section{Parametrix COnstruction}

This highly technical section contains detailed proofs of a number of facts and estimates needed to provide the construction of the fundamental solution $p_{t, s}(x, y)$, which was explained earlier in Section 3. The construction demands many auxiliary results, in particular key estimates of the zero-order approximation term $p_{t, s}^{(0)}(x, y)$ and the kernel $q_{t, s}^{(0)}(x, y)$ contained in Lemma 5.8 and Lemma 5.10, respectively.

In this section we adopt the convention that constants denoted by $c\left(\right.$ or $\left.c_{1}, c_{2}, \ldots\right)$ may change their value from one use to the next. Unless is explicitly stated otherwise, we understand that constants denoted by $c$ (or $c_{1}, c_{2}, \ldots$ ) depend only on $d, \alpha, \beta, \gamma_{1}, \gamma_{2}, \gamma_{3}$, $C_{1}, \ldots, C_{7}$. We also understand that they may depend on on the choice of the constant $\varepsilon$. We write $c=c(a, b, \ldots)$ when $c$ depends on the above constants and additionally on $a, b$, .... For a square matrix $A$ we denote by $|A|$ its standard operator norm. The standard inner product for $x, y \in \mathbb{R}^{d}$ we denote by $x y$. 
Remark 5.1. Our choice of $p_{t, s}^{(0)}(x, y)$ and the kernel $q_{t, s}^{(0)}(x, y)$ will depend on the given value of $\varepsilon>0$. In order to have required bounds involving these objects, we have to impose a restriction on $\varepsilon$. Namely throughout the rest of the paper we assume that $\varepsilon \leq \varepsilon_{0}$, with $\varepsilon_{0}$ defined below.

In the case $(\mathrm{A})$ we set

$\varepsilon_{0}=\min \left\{\frac{\gamma_{1} \alpha}{2(d+3) \beta}, \frac{\gamma_{2} \alpha}{2(d+3)}, \frac{\gamma_{1} /\left(\beta\left(1+\gamma_{1}\right)\right)}{2+2 / \alpha+\gamma_{1} /\left(\beta\left(1+\gamma_{1}\right)\right)}, \frac{\gamma_{3}-1}{\gamma_{3}-1+\beta(1+(d+1) / \alpha)}\right\}$, while in the case $(\mathrm{B})$ we pick

$$
\begin{aligned}
\varepsilon_{0}=\min \left\{\frac{\left(1+\gamma_{1}\right) / \beta-1 / \alpha}{2(d+3) / \alpha}, \frac{\gamma_{2}-(1 / \alpha-1 / \beta)}{2(d+3) / \alpha}, \frac{1 / \beta-1 /\left(\left(1+\gamma_{1}\right) \alpha\right)}{2+2 / \alpha+1 / \beta-1 /\left(\left(1+\gamma_{1}\right) \alpha\right)},\right. \\
\left.\frac{\gamma_{3}-\beta / \alpha}{\gamma_{3}+\beta(1+d / \alpha)}\right\} .
\end{aligned}
$$

Due to our assumptions $\varepsilon_{0}$ is positive.

For $i \in\{1, \ldots, d\}, u>0$ put

$$
R_{u}^{(i)}=R_{u}^{(i)}(\varepsilon)=h_{i}^{-1}\left(\frac{1}{u^{1-\varepsilon}}\right) .
$$

Let $g_{u}^{(i)}=g_{u}^{(i, \varepsilon)}$ be the truncated density corresponding to $\psi_{i}$ according to the truncation procedure described in Section 4

For any $u>0, x \in \mathbb{R}^{d}$ define

$$
G_{u}(x)=g_{u}^{(1)}\left(x_{1}\right) g_{u}^{(2)}\left(x_{2}\right) \cdots g_{u}^{(d)}\left(x_{d}\right) .
$$

Let $h_{\max }^{-1}(r)=\max _{j} h_{j}^{-1}(r)$ and let $h_{\min }^{-1}(r)=\min _{j} h_{j}^{-1}(r)$. Let $M_{u}$ be a diagonal $d$ - dimensional square matrix with the diagonal $8\left(R_{u}^{(1)}, \ldots R_{u}^{(d)}\right)$. The multiplier 8 is only for the notational convenience. Note that $\left|M_{u}\right|=8 \max _{i} R_{u}^{(i)}=8 h_{\max }^{-1}\left(u^{-1+\varepsilon}\right)$ and $\left|M_{u}^{-1}\right|=\frac{1}{8} \max _{i} \frac{1}{R_{u}^{(i)}}=\frac{1}{8} \frac{1}{h_{\min }^{-1}\left(u^{-1+\varepsilon}\right)}$.

We recall that for $u>0$ we defined

$$
\kappa(u)=\left(u, h_{1}(1), \ldots, h_{d}(1), h_{1}^{-1}(1), \ldots, h_{d}^{-1}(1), h_{1}^{-1}(1 / u), \ldots, h_{d}^{-1}(1 / u)\right) .
$$

Observe that for every $\tau>0$

$$
\frac{1}{c} u^{(\varepsilon-1) / \beta} \leq\left|M_{u}^{-1}\right| \leq c u^{(\varepsilon-1) / \alpha}, 0<u \leq \tau,
$$

and

$$
\frac{1}{c} u^{(1-\varepsilon) / \alpha} \leq\left|M_{u}\right| \leq c u^{(1-\varepsilon) / \beta}, 0<u \leq \tau,
$$

where $c$ depends also on $\tau$ trough the vector $\kappa(\tau)$, that is in our notation $c=c(\kappa(\tau))$. This follows from Lemma 4.2. Throughout the whole section we set

$$
\tau_{0}=\min _{k \leq d}\left\{\left(h_{k}(1) \vee 1\right)^{-\frac{1}{1-\varepsilon}}\right\} .
$$

Then the constant $c$ in (67) and (68) depends on the vector $\bar{h}=\left(h_{1}(1), \ldots, h_{d}(1)\right)$, if $\tau \leq \tau_{0}$, in our convention we write $c=c(\bar{h})$. Again, this follows from Lemma 4.2.

The following lemma follows easily from Corollary 4.7 and Lemma 4.10.

Lemma 5.2. Fix $\tau>0$. For any $u \in(0, \tau], x \in \mathbb{R}^{d}$ we have

$$
\begin{gathered}
c^{-1} \prod_{i=1}^{d} \frac{1}{h_{i}^{-1}(1 / u)} \leq G_{u}(0) \leq c \prod_{i=1}^{d} \frac{1}{h_{i}^{-1}(1 / u)}, \\
G_{u}(x) \leq c G_{u}(0) e^{-\left|x M_{u}^{-1}\right|},
\end{gathered}
$$




$$
\begin{gathered}
\left|\frac{\partial}{\partial x_{i}} G_{u}(x)\right| \leq c G_{u}(0) \frac{1}{h_{i}^{-1}(1 / u)} u^{-\varepsilon} e^{-\left|x M_{u}^{-1}\right|}, \\
\left|\frac{\partial^{2}}{\partial x_{i} \partial x_{j}} G_{u}(x)\right| \leq c G_{u}(0) \frac{1}{h_{i}^{-1}(1 / u) h_{j}^{-1}(1 / u)} u^{-2 \varepsilon} e^{-\left|x M_{u}^{-1}\right|},
\end{gathered}
$$

where the constant $c$ depends additionally on $\tau$, that is $c=c(\kappa(\tau))$. If $\tau \leq \tau_{0}$, then $c=c(\bar{h})$.

Proof. The first estimate follows directly from Corollary 4.7.

All the remaining estimates follow directly from Lemma 4.10 and the observation

$$
\sum_{j=1}^{d} \frac{\left|x_{j}\right|}{8 R_{u}^{(i)}} \geq\left[\sum_{j=1}^{d}\left(\frac{\left|x_{j}\right|}{8 R_{u}^{(i)}}\right)^{2}\right]^{1 / 2}=\left|x M_{u}^{-1}\right|
$$

By (47) and the above lemma we obtain the following corollary.

Corollary 5.3. Fix $\tau>0$. For any $u \in(0, \tau]$

$$
G_{u \lambda}(0) \leq c \lambda^{-d / \alpha} G_{u}(0), \quad \lambda \leq 1
$$

where $c=c(\kappa(\tau))$. If $\tau \leq \tau_{0}$, then $c=c(\bar{h})$.

For $0 \leq t<s$ and $x, w, y \in \mathbb{R}^{d}$ put

$$
p_{t, s}^{x}(w)=\frac{1}{\left|\operatorname{det} A_{s}(x)\right|} G_{s-t}\left(w\left(A_{s}^{-1}(x)\right)^{T}\right) .
$$

and

$$
L_{t, s}^{y} f(x)=\sum_{k=1}^{d} \text { P.V. } \int_{|u|<R_{s-t}^{(k)}}\left[f\left(x+u e_{k} A_{s}^{T}(y)\right)-f(x)\right] \nu_{k}(d u) .
$$

By (66), for any $0 \leq t<s$ and $w, y \in \mathbb{R}^{d}$, we have

$$
\left(\frac{\partial}{\partial t}+L_{t, s}^{y}\right) p_{t, s}^{y}(w)=0
$$

We choose our zero-order approximation $p_{t, s}^{(0)}$ in parametrix construction as

$$
p_{t, s}^{(0)}(x, y)=p_{t, s}^{y}(x-y)
$$

Before we come to crucial estimates of $q_{t, s}^{(0)}$ (defined in Section 3.1) we need to show some auxiliary results on $p_{t, s}^{w}$.

Let

$$
\begin{aligned}
\|A\|= & \max \left(\sup _{t>0, x \in \mathbb{R}^{d}}\left|A_{t}^{T}(x)\right|, \sup _{t>0, x \in \mathbb{R}^{d}}\left|A_{t}^{-1}(x)\right|, \sup _{t>0, x, y \in \mathbb{R}^{d}, x \neq y} \frac{\left|A_{t}^{T}(x)-A_{t}^{T}(y)\right|}{|x-y|^{\gamma_{1}}},\right. \\
& \left.\sup _{t>0, x, y \in \mathbb{R}^{d}, x \neq y} \frac{\left|\left(A_{t}^{T}(x)\right)^{-1}-\left(A_{t}^{T}(y)\right)^{-1}\right|}{|x-y|^{\gamma_{1}}}, \sup _{s>t>0, x \in \mathbb{R}^{d},} \frac{\left|\left(A_{t}^{T}(x)\right)^{-1}-\left(A_{s}^{T}(x)\right)^{-1}\right|}{(s-t)^{\gamma_{2}}}\right) .
\end{aligned}
$$

It is clear that $\|A\| \geq 1$. Note that $\|A\|$ may be bounded from above by a constant which depends only on $d, C_{3}, \ldots, C_{6}$. Using standard calculations and the conditions (8, 9. 10, 11) we have

$$
\|A\| \leq\left(C_{3}+C_{5}+C_{6}\right) d+\frac{C_{3}^{d-1}}{C_{4}} d+\left(C_{5}+C_{6}\right) \frac{C_{3}^{d-1}}{C_{4}} d^{2} .
$$

From Lemma 5.2, Lemma 4.2 and (69) we easily get the following corollary. 
Corollary 5.4. Let $\tau>0$. Then there is a constant $c=c(\kappa(\tau))$ such that for $0<s-t \leq \tau$ and $y, w \in \mathbb{R}^{d}$

$$
\begin{aligned}
& \left|p_{t, s}^{y}(w)\right| \leq c G_{s-t}(0) e^{-\left|w\left(A_{s}^{-1}(y)\right)^{T} M_{s-t}^{-1}\right|}, \\
& \left|p_{t, s}^{y}(w)\right| \leq c G_{s-t}(0) e^{-\frac{1}{|A|} \frac{|w|}{\left|M_{s-t}\right|}}, \\
& \left|\nabla p_{t, s}^{y}(w)\right| \leq c\left|M_{s-t}^{-1}\right|(s-t)^{-\varepsilon(1+1 / \alpha)} G_{s-t}(0) e^{-\left|w\left(A_{s}^{-1}(y)\right)^{T} M_{s-t}^{-1}\right|}, \\
& \left|\nabla^{2} p_{t, s}^{y}(w)\right| \leq c\left|M_{s-t}^{-1}\right|^{2}(s-t)^{-\varepsilon(2+2 / \alpha)} G_{s-t}(0) e^{-\left|w\left(A_{s}^{-1}(y)\right)^{T} M_{s-t}^{-1}\right|} \text {, } \\
& \left|\nabla^{2} p_{t, s}^{y}(w)\right| \leq c\left|M_{s-t}^{-1}\right|^{2}(s-t)^{-\varepsilon(2+2 / \alpha)} G_{s-t}(0) e^{-\frac{1}{|| A||} \frac{|w|}{\left|M_{s-t}\right|}} .
\end{aligned}
$$

If $\tau \leq \tau_{0}$, then $c=c(\bar{h})$.

Proof. We provide the proof only for (774) and (75) since the other estimates can be shown in a similar fashion. Applying Lemma 5.2 and (69) we obtain

$$
\left|\nabla^{2} p_{t, s}^{y}(w)\right| \leq c G_{s-t}(0) \frac{1}{\left(h_{\min }^{-1}\left((s-t)^{-1}\right)\right)^{2}}(s-t)^{-2 \varepsilon} e^{-\left|w\left(A_{s}^{-1}(y)\right)^{T} M_{s-t}^{-1}\right|} .
$$

Next, by (45),

$$
\frac{1}{\left|M_{s-t}^{-1}\right| h_{\min }^{-1}\left((s-t)^{-1}\right)}=\frac{h_{\min }^{-1}\left((s-t)^{\varepsilon-1}\right)}{8 h_{\min }^{-1}\left((s-t)^{-1}\right)} \leq c(s-t)^{-\varepsilon / \alpha},
$$

which completes the proof of (74).

Since $\left|w\left(A_{s}^{-1}(y)\right)^{T} M_{s-t}^{-1}\right| \geq \frac{1}{\| A||} \frac{|w|}{\left|M_{s-t}\right|}$ the estimate (74) yields (175).

The following lemma is a simple consequence of the change of variable formula, hence its proof is omitted.

Lemma 5.5. Let $\rho>0, x \in \mathbb{R}^{d}, 0 \leq t<s<\infty$. There is a constant $c=c(\rho)$ such that

$$
\int_{\mathbb{R}^{d}}|x-y|^{\rho} e^{-\left|(x-y)\left(A_{s}^{-1}(x)\right)^{T} M_{s-t}^{-1}\right|} d y \leq c\left|M_{s-t}\right|^{\rho} \operatorname{det}\left(M_{s-t}\right) .
$$

Lemma 5.6. Let $x, y \in \mathbb{R}^{d}, 0 \leq t<s$ and $\delta>0$. Let

$$
\left.\xi=y-x+\theta u e_{k} A_{t}^{T}(x)+(1-\theta) u e_{k} A_{s}^{T}(y)\right)+\lambda U_{t}\left(x, e_{k} u\right),|u| \leq R_{s-t}^{(k)},
$$

where $0 \leq \theta, \lambda \leq 1$.

$$
\begin{aligned}
& \text { If }|x-y|^{1+\gamma_{1} \leq} \leq \frac{(s-t)^{\delta}}{\left|M_{s-t}^{-1}\right|} \text { and } 0<s-t \leq \tau \text {, then } \\
& \qquad\left|\xi\left(A_{s}^{T}(y)\right)^{-1} M_{s-t}^{-1}-(y-x)\left(A_{s}^{T}(x)\right)^{-1} M_{s-t}^{-1}\right| \leq c .
\end{aligned}
$$

The contant $c=c(\delta, \kappa(\tau))$. If $\tau \leq \tau_{0}$, then $c=c(\delta, \bar{h})$.

Proof. We denote $z=y-x, U=U_{t}\left(x, e_{k} u\right), R^{(k)}=R_{s-t}^{(k)}$ and $M=M_{s-t}$. It is clear that it is enough to consider the case when $\theta=1$ or $\theta=0$. We provide the argument if $\theta=1$ since the case $\theta=0$ is similar, if not easier. Let $\xi=z+u e_{k} A_{t}^{T}(x)$. Noting that $u e_{k} M^{-1}=\left(u / R^{(k)}\right) e_{k}$, we obtain

$$
\begin{aligned}
& \left|\xi\left(A_{s}^{T}(y)\right)^{-1} M^{-1}-z\left(A_{s}^{T}(x)\right)^{-1} M^{-1}\right| \\
& =\mid z\left[\left(A_{s}^{T}(y)\right)^{-1}-\left(A_{s}^{T}(x)\right)^{-1}\right] M^{-1}+u e_{k}\left[A_{t}^{T}(x)-A_{s}^{T}(y)\right]\left(A_{s}^{T}(y)\right)^{-1} M^{-1} \\
& +u e_{k} M^{-1}+U\left(A_{s}^{T}(y)\right)^{-1} M^{-1} \mid \\
& \leq|| A|||z|^{1+\gamma_{1}}\left|M^{-1}\right|+|| A|||u||z|^{\gamma_{1}}\left|M^{-1}\right|+|u| / R^{(k)}+C_{7}|| A||\left|M^{-1}\right||u|^{\gamma_{3}} \\
& \leq|| A \|\left(|z|^{1+\gamma_{1}}\left|M^{-1}\right|+R^{(k)}|z|^{\gamma_{1}}\left|M^{-1}\right|+1+C_{7}\left|M^{-1}\right|\left(R^{(k)}\right)^{\gamma_{3}}\right)
\end{aligned}
$$


In the case (A) ( when $h_{1}=\cdots=h_{d}$ ) we have $R^{(k)}\left|M^{-1}\right|=1 / 8$, hence, by (68),

$|z|^{1+\gamma_{1}}\left|M^{-1}\right|+R^{(k)}|z|^{\gamma_{1}}\left|M^{-1}\right|+1+C_{7}\left|M^{-1}\right|\left(R^{(k)}\right)^{\gamma_{3}} \leq(s-t)^{\delta}+|z|^{\gamma_{1}}+1+C_{7}\left|M^{-1}\right|^{1-\gamma_{3}} \leq c$, with $c=c(\delta, \kappa(\tau))$.

In the case (B), by (67) and (68),

$$
\begin{aligned}
& \left.\left|R^{(k)}\right| z\right|^{\gamma_{1}}|| M^{-1}\left|\leq c(s-t)^{(1-\varepsilon) / \beta}\left(\frac{(s-t)^{\delta}}{8\left|M^{-1}\right|}\right)^{\frac{\gamma_{1}}{\gamma_{1}+1}}\right| M^{-1} \mid \\
& =c(s-t)^{(1-\varepsilon) / \beta+\delta \frac{\gamma_{1}}{\gamma_{1}+1}}\left|M^{-1}\right|^{\frac{1}{\gamma_{1}+1}} \\
& \leq c(s-t)^{(1-\varepsilon) / \beta+\delta \frac{\gamma_{1}}{\gamma_{1}+1}}(s-t)^{\frac{-1+\varepsilon}{\left.\gamma_{1}+1\right) \alpha}}=c(s-t)^{\delta_{0}},
\end{aligned}
$$

where $\delta_{0}=\delta \frac{\gamma_{1}}{\gamma_{1}+1}+(1-\varepsilon)\left(\frac{1}{\beta}-\frac{1}{\left(\gamma_{1}+1\right) \alpha}\right)>0$. Moreover, again by (67) and (68),

$$
\left|M^{-1}\right|\left(R^{(k)}\right)^{\gamma_{3}} \leq c(s-t)^{(1-\varepsilon)\left(\frac{\gamma_{3}}{\beta}-\frac{1}{\alpha}\right)} .
$$

This implies that

$$
\begin{aligned}
& |z|^{1+\gamma_{1}}\left|M^{-1}\right|+R^{(k)}|z|^{\gamma_{1}}\left|M^{-1}\right|+1+\eta_{5}\left|M^{-1}\right|\left(R^{(k)}\right)^{\gamma_{3}} \\
& \leq c(s-t)^{\delta}+c(s-t)^{\delta_{0}}+1+c(s-t)^{(1-\varepsilon)\left(\frac{\gamma_{3}}{\beta}-\frac{1}{\alpha}\right)} \leq c,
\end{aligned}
$$

with $c=c(\delta, \kappa(\tau))$. Hence, in both cases, the proof of (76) is completed.

For any $x \in \mathbb{R}^{d}, \delta>0$ let

$$
D(\delta, x)=\left\{w \in \mathbb{R}^{d}:|x-w|^{1+\gamma_{1}} \leq \frac{(s-t)^{\delta}}{\left|M_{s-t}^{-1}\right|}\right\} .
$$

Lemma 5.7. Fix $\tau>0$ and $\delta>0$. For any $x \in \mathbb{R}^{d}, y \in D(\delta, x), 0<s-t \leq \tau$ we have

$$
\begin{aligned}
& \left|G_{s-t}\left((x-y)\left(A_{s}^{T}(y)\right)^{-1}\right)-G_{s-t}\left((x-y)\left(A_{s}^{T}(x)\right)^{-1}\right)\right| \\
& \leq c(s-t)^{\delta-\varepsilon(1+1 / \alpha)} G_{s-t}(0) e^{-\left|(x-y)\left(A_{s}^{-1}(x)\right)^{T} M_{s-t}^{-1}\right|}
\end{aligned}
$$

and

$$
\int_{D(\delta, x)}\left|G_{s-t}\left((x-y)\left(A_{s}^{T}(y)\right)^{-1}\right)-G_{s-t}\left((x-y)\left(A_{s}^{T}(x)\right)^{-1}\right)\right| d y \leq c(s-t)^{\delta-(d+3) \varepsilon / \alpha} .
$$

The contant $c=c(\delta, \kappa(\tau))$. If $\tau \leq \tau_{0}$, then $c=c(\delta, \bar{h})$.

Proof. We have

$$
\begin{aligned}
G_{s-t}\left((x-y)\left(A_{s}^{T}(y)\right)^{-1}\right) & =G_{s-t}\left((x-y)\left(A_{s}^{T}(x)\right)^{-1}\right) \\
& +\nabla G_{s-t}(\xi)\left[(x-y)\left(\left(A_{s}^{T}(y)\right)^{-1}-\left(A_{s}^{T}(x)\right)^{-1}\right)\right],
\end{aligned}
$$

where $\xi=\theta(x-y)\left(A_{s}^{T}(x)\right)^{-1}+(1-\theta)(x-y)\left(A_{s}^{T}(y)\right)^{-1}, 0 \leq \theta \leq 1$. Next, we observe that

$$
\left|(x-y)\left(A_{s}^{T}(y)\right)^{-1}-(x-y)\left(A_{s}^{T}(x)\right)^{-1}\right| \leq|| A|||x-y|^{1+\gamma_{1}} \leq(s-t)^{\delta}\|A\| \frac{1}{\left|M_{s-t}^{-1}\right|} .
$$

Hence,

$$
\left|\xi M_{s-t}^{-1}-(x-y)\left(A_{s}^{T}(x)\right)^{-1} M_{s-t}^{-1}\right| \leq(s-t)^{\delta}\left|M_{s-t}^{-1}\right||| A||\left|M_{s-t}^{-1}\right|^{-1}=(s-t)^{\delta}\|A\| .
$$

This implies, by Lemma 5.2, that

$$
\left|\nabla G_{s-t}(\xi)\right| \leq c \frac{1}{h_{\min }^{-1}(1 /(s-t))}(s-t)^{-\varepsilon} G_{s-t}(0) e^{-\left|(x-y)\left(A_{s}^{-1}(x)\right)^{T} M_{s-t}^{-1}\right|},
$$


which together with (79) yield

$$
\begin{aligned}
& \left|\nabla G_{s-t}(\xi)\left[(x-y)\left(\left(A_{s}^{T}(y)\right)^{-1}-\left(A_{s}^{T}(x)\right)^{-1}\right)\right]\right| \\
& \leq c(s-t)^{\delta} \frac{1}{\left|M_{s-t}^{-1}\right|} \frac{1}{h_{\min }^{-1}(1 /(s-t))}(s-t)^{-\varepsilon} G_{s-t}(0) e^{-\left|(x-y)\left(A_{s}^{-1}(x)\right)^{T} M_{s-t}^{-1}\right|} \\
& \leq c(s-t)^{\delta}(s-t)^{-\varepsilon(1+1 / \alpha)} G_{s-t}(0) e^{-\left|(x-y)\left(A_{s}^{-1}(x)\right)^{T} M_{s-t}^{-1}\right|},
\end{aligned}
$$

since, by Corollary 4.3 ,

$$
\frac{1}{\left|M_{s-t}^{-1}\right|} \frac{1}{h_{\min }^{-1}(1 /(s-t))}=8 \frac{h_{\min }^{-1}\left((s-t)^{\varepsilon-1}\right)}{h_{\min }^{-1}\left((s-t)^{-1}\right)} \leq c(s-t)^{-\varepsilon / \alpha} .
$$

The proof of (77) is completed.

Applying (77) and Lemma 5.5 we get

$$
\begin{aligned}
& \int_{D(\delta, x)}\left|G_{s-t}\left((x-y)\left(A_{s}^{T}(y)\right)^{-1}\right)-G_{s-t}\left((x-y)\left(A_{s}^{T}(x)\right)^{-1}\right)\right| d y \\
& \leq \operatorname{cdet}\left(M_{s-t}\right) G_{s-t}(0)(s-t)^{\delta-\varepsilon-\varepsilon / \alpha} .
\end{aligned}
$$

Next, by Lemma 5.2 and Corollary 4.3 .

$$
\operatorname{det}\left(M_{s-t}\right) G_{s-t}(0) \leq c \prod_{k=1}^{d} \frac{h_{k}^{-1}\left((s-t)^{\varepsilon-1}\right)}{h_{k}^{-1}\left((s-t)^{-1}\right)} \leq c(s-t)^{-d \varepsilon / \alpha},
$$

which implies (78).

Lemma 5.8. Fix $\tau>0$. There exists $c(\kappa(\tau))=c>0$ such that for any $0<s-t \leq \tau$ and $x \in \mathbb{R}^{d}$ we have

$$
\int_{\mathbb{R}^{d}} p_{t, s}^{y}(x-y) d y \leq c .
$$

Moreover, for any $0<s-t \leq \tau$ and $x \in \mathbb{R}^{d}$ we have

$$
\sup _{y \in \mathbb{R}^{d}}\left|p_{t, s}^{y}(x-y)-p_{t, s}^{x}(x-y)\right| \leq c G_{s-t}(0)(s-t)^{\varepsilon}
$$

and

$$
\int_{\mathbb{R}^{d}}\left|p_{t, s}^{y}(x-y)-p_{t, s}^{x}(x-y)\right| d y \leq c(s-t)^{\varepsilon} .
$$

If $\tau \leq \tau_{0}$, then $c=c(\bar{h})$.

Proof. Let $\delta>0$ and $z$ be $x$ or $y$. For $y \in D^{c}(\delta, x)$ we use (72) and Lemma 4.2 to have

$$
p_{t, s}^{z}(x-y) \leq c(s-t)^{-d / \alpha} \exp \left(-\frac{|x-y|}{|| A||\left|M_{s-t}\right|}\right) .
$$

In the case (A) we have $\left|M_{s-t}^{-1}\right|^{-1}=\left|M_{s-t}\right|$. Hence, by (68),

$$
\begin{aligned}
\frac{|x-y|}{\left|M_{s-t}\right|} & \geq \frac{\left[(s-t)^{\delta}\left|M_{s-t}\right|\right]^{\frac{1}{1+\gamma_{1}}}}{\left|M_{s-t}\right|}=\left[(s-t)^{\delta}\left|M_{s-t}\right|^{-\gamma_{1}}\right]^{\frac{1}{1+\gamma_{1}}} \\
& \geq c\left[(s-t)^{\delta-(1-\varepsilon) \frac{\gamma_{1}}{\beta}}\right]^{\frac{1}{1+\gamma_{1}}} .
\end{aligned}
$$

In the case $(\mathrm{A})$ we choose $\delta=\frac{(1-2 \varepsilon) \gamma_{1}}{\beta}<\frac{(1-\varepsilon) \gamma_{1}}{\beta}$. Then clearly the exponent at $s-t$ is negative.

Next, we observe that in the case (B), by (67) and (68),

$$
\frac{|x-y|}{\left|M_{s-t}\right|} \geq \frac{\left[(s-t)^{\delta}\left|M_{s-t}^{-1}\right|^{-1}\right]^{\frac{1}{1+\gamma_{1}}}}{\left|M_{s-t}\right|} \geq c(s-t)^{\frac{\delta+(1-\varepsilon) / \alpha-(1-\varepsilon)\left(1+\gamma_{1}\right) / \beta}{1+\gamma_{1}}} .
$$


In the case $(\mathrm{B})$ we choose $\delta=(1-2 \varepsilon)\left[\left(1+\gamma_{1}\right) / \beta-1 / \alpha\right]<(1-\varepsilon)\left[\left(1+\gamma_{1}\right) / \beta-1 / \alpha\right]$. Then clearly the exponent at $s-t$ is negative.

Hence, in both cases, we find $c=c(\kappa(\tau))$ such that for $y \in D^{c}(\delta, x)$

$$
p_{t, s}^{z}(y-x) \leq c(s-t)^{-d / \alpha} \exp \left(-\frac{|x-y|}{|| A||\left|M_{s-t}\right|}\right) \leq c(s-t)^{\varepsilon} .
$$

Similarly, we find $c=c(\kappa(\tau))$ such that

$$
\int_{D^{c}(\delta, x)} p_{t, s}^{z}(y-x) d y \leq c(s-t)^{-d / \alpha} \int_{D^{c}(\delta, x)} \exp \left(-\frac{|x-y|}{|| A||\left|M_{s-t}\right|}\right) d y \leq c(s-t)^{\varepsilon} .
$$

For any $x \in \mathbb{R}^{d}, 0<s-t \leq \tau$ we have, by (78),

$$
\begin{aligned}
\int_{D(\delta, x)} p_{t, s}^{y}(x-y) & \leq \frac{1}{C_{4}} \int_{D(\delta, x)} G_{s-t}\left((x-y)\left(A_{s}^{T}(y)\right)^{-1}\right) d y \\
& \leq \frac{1}{C_{4}} \int_{D(\delta, x)}\left|G_{s-t}\left((x-y)\left(A_{s}^{T}(y)\right)^{-1}\right)-G_{s-t}\left((x-y)\left(A_{s}^{T}(x)\right)^{-1}\right)\right| d y \\
& +\frac{1}{C_{4}} \int_{D(\delta, x)} G_{s-t}\left((x-y)\left(A_{s}^{T}(x)\right)^{-1}\right) d y \\
& \leq c+c(s-t)^{\delta-(d+3) \varepsilon / \alpha}
\end{aligned}
$$

We also have

$$
\begin{aligned}
& \left|p_{t, s}^{y}(x-y)-p_{t, s}^{x}(x-y)\right| \\
& =\mid \frac{1}{\left|\operatorname{det}\left(A_{s}(y)\right)\right|} G_{s-t}\left((x-y)\left(A_{s}^{T}(y)\right)^{-1}\right)-\frac{1}{\left|\operatorname{det}\left(A_{s}(x)\right)\right|} G_{s-t}\left((x-y)\left(A_{s}^{T}(y)\right)^{-1}\right) \\
& +\frac{1}{\left|\operatorname{det}\left(A_{s}(x)\right)\right|} G_{s-t}\left((x-y)\left(A_{s}^{T}(y)\right)^{-1}\right)-\frac{1}{\left|\operatorname{det}\left(A_{s}(x)\right)\right|} G_{s-t}\left((x-y)\left(A_{s}^{T}(x)\right)^{-1}\right) \mid \\
& \leq c|x-y|^{\gamma_{1}} G_{s-t}\left((x-y)\left(A_{s}^{T}(y)\right)^{-1}\right) \\
& +c\left|G_{s-t}\left((x-y)\left(A_{s}^{T}(y)\right)^{-1}\right)-G_{s-t}\left((x-y)\left(A_{s}^{T}(x)\right)^{-1}\right)\right|
\end{aligned}
$$

Note that for any $x \in \mathbb{R}^{d}, y \in D(\delta, x)$ and $0<s-t \leq \tau$ we have $|x-y|^{\gamma_{1}} \leq c(s-t)^{\delta+(1-\varepsilon) / \beta}$. It follows that for any $x \in \mathbb{R}^{d}$ and $0<s-t \leq \tau$

$$
\int_{D(\delta, x)}\left|p_{t, s}^{y}(x-y)-p_{t, s}^{x}(x-y)\right| d y \leq c(s-t)^{\delta+(1-\varepsilon) / \beta}+c(s-t)^{\delta-(d+3) \varepsilon / \alpha} .
$$

Recall that in the case (A) we picked $\delta=\frac{(1-2 \varepsilon) \gamma_{1}}{\beta}$. Since $\varepsilon \leq \varepsilon_{0} \leq \frac{\gamma_{1} \alpha}{2(d+3) \beta}$ we have $\frac{(d+3) \varepsilon}{\alpha} \leq \frac{\gamma_{1}}{2 \beta}=\frac{\delta}{2(1-2 \varepsilon)}<\delta$. Hence

$$
\delta-\frac{(d+3) \varepsilon}{\alpha} \geq \delta\left(1-\frac{1}{2(1-2 \varepsilon)}\right) \geq(1-4 \varepsilon) \frac{(d+3) \varepsilon}{\alpha} \geq \varepsilon,
$$

since $\varepsilon \leq 1 / 8$.

Recall that in the case (B) we picked $\delta=(1-2 \varepsilon)\left(\left(1+\gamma_{1}\right) / \beta-1 / \alpha\right)$. Since $\varepsilon \leq \varepsilon_{0} \leq$ $\frac{\left(1+\gamma_{1}\right) / \beta-1 / \alpha}{2(d+3) / \alpha}$ we obtain $\frac{(d+3) \varepsilon}{\alpha} \leq \frac{\left(1+\gamma_{1}\right) / \beta-1 / \alpha}{2}=\frac{\delta}{2(1-2 \varepsilon)}<\delta$, since $\varepsilon \leq 1 / 8$. Hence, as in the case $(\mathrm{A})$ we obtain

$$
\delta-\frac{(d+3) \varepsilon}{\alpha} \geq \delta\left(1-\frac{1}{2(1-2 \varepsilon)}\right) \geq(1-4 \varepsilon) \frac{(d+3) \varepsilon}{\alpha} \geq \varepsilon .
$$

Now (86), (87) imply (81). By (77) and (85) we get (821). (86), (88) imply (83). 
Lemma 5.9. There exist $c=c(\bar{h})$ such that for any $0 \leq t<s$ and $x \in \mathbb{R}^{d}$ we have $\sup _{y \in \mathbb{R}^{d}}\left|G_{s-t}\left((x-y)\left(A_{s}^{T}(x)\right)^{-1}\right)-G_{s-t}\left((x-y)\left(A_{t}^{T}(x)\right)^{-1}\right)\right| \leq c G_{s-t}(0)(s-t)^{\varepsilon}$. and

$$
\int_{\mathbb{R}^{d}}\left|G_{s-t}\left((x-y)\left(A_{s}^{T}(x)\right)^{-1}\right)-G_{s-t}\left((x-y)\left(A_{t}^{T}(x)\right)^{-1}\right)\right| d y \leq c(s-t)^{\varepsilon} .
$$

Proof. We first prove the lemma under the assumption $0<s-t \leq \tau_{0}$. Let $\delta>0$. Put

$$
\tilde{D}(\delta, x)=\left\{w:|x-w| \leq \frac{(s-t)^{\delta-\gamma_{2}}}{\left|M_{s-t}^{-1}\right|}\right\} .
$$

First, we consider $y \in \tilde{D}(\delta, x)$. Then we have

$$
\left|(x-y)\left(A_{s}^{T}(x)\right)^{-1}-(x-y)\left(A_{t}^{T}(x)\right)^{-1}\right| \leq\|A\||x-y|(s-t)^{\gamma_{2}} \leq \frac{(s-t)^{\delta}\|A\|}{\left|M_{s-t}^{-1}\right|} .
$$

By the same arguments as in the proof of Lemma 5.7, we get

$$
\begin{aligned}
& \left|G_{s-t}\left((x-y)\left(A_{s}^{T}(x)\right)^{-1}\right)-G_{s-t}\left((x-y)\left(A_{t}^{T}(x)\right)^{-1}\right)\right| \\
& \leq c(s-t)^{\delta-\varepsilon(1+1 / \alpha)} G_{s-t}(0) e^{-\left|(x-y)\left(A_{s}^{-1}(x)\right)^{T} M_{s-t}^{-1}\right|} .
\end{aligned}
$$

and

$$
\int_{\tilde{D}(\delta, x)}\left|G_{s-t}\left((x-y)\left(A_{s}^{T}(x)\right)^{-1}\right)-G_{s-t}\left((x-y)\left(A_{t}^{T}(x)\right)^{-1}\right)\right| d y \leq c(s-t)^{\delta-(d+3) \varepsilon / \alpha} .
$$

Next, we estimate the expression $\left|G_{s-t}\left((x-y)\left(A_{s}^{T}(x)\right)^{-1}\right)-G_{s-t}\left((x-y)\left(A_{t}^{T}(x)\right)^{-1}\right)\right|$ for $y \in \tilde{D}^{c}(\delta, x)$. In the case (A) we have $\left|M_{s-t}^{-1}\right|^{-1}=\left|M_{s-t}\right|$. Hence for $y \in \tilde{D}^{c}(\delta, x)$

$$
\frac{|x-y|}{\left|M_{s-t}\right|} \geq(s-t)^{\delta-\gamma_{2}} \text {. }
$$

In the case (A) we will assume that $\delta<\gamma_{2}$.

In the case (B), by (67) and (68), for $y \in \tilde{D}^{c}(\delta, x)$

$$
\frac{|x-y|}{\left|M_{s-t}\right|} \geq \frac{(s-t)^{\delta-\gamma_{2}}}{\left|M_{s-t}^{-1}\right|\left|M_{s-t}\right|} \geq c(s-t)^{\delta-\gamma_{2}+(1-\varepsilon)\left(\frac{1}{\alpha}-\frac{1}{\beta}\right)} .
$$

In the case (B) we will assume that $\delta<\gamma_{2}-\left(\frac{1}{\alpha}-\frac{1}{\beta}\right)$.

By the same arguments as in the proof of Lemma 5.8 we find $c=c(\bar{h})$ such that for $y \in \tilde{D}^{c}(\delta, x)$

$$
\left|G_{s-t}\left((x-y)\left(A_{s}^{T}(x)\right)^{-1}\right)-G_{s-t}\left((x-y)\left(A_{t}^{T}(x)\right)^{-1}\right)\right| \leq c(s-t)^{\varepsilon} \leq c G_{s-t}(0)(s-t)^{\varepsilon}
$$

and

$$
\int_{\tilde{D}^{c}(\delta, x)}\left|G_{s-t}\left((x-y)\left(A_{s}^{T}(x)\right)^{-1}\right)-G_{s-t}\left((x-y)\left(A_{t}^{T}(x)\right)^{-1}\right)\right| d y \leq c(s-t)^{\varepsilon} .
$$

In the case $(\mathrm{A})$ we pick $\delta=(1-\varepsilon) \gamma_{2}$. Since $\varepsilon \leq \varepsilon_{0} \leq \frac{\gamma_{2} \alpha}{2(d+3)} \leq 1 / 4$ we have $\frac{(d+3) \varepsilon}{\alpha} \leq$ $\frac{\gamma_{2}}{2}=\delta / 2(1-\varepsilon)$. Hence

$$
\delta-\frac{(d+3) \varepsilon}{\alpha} \geq \delta\left(1-\frac{1}{2(1-\varepsilon)}\right) \geq(1-2 \varepsilon) \frac{(d+3) \varepsilon}{\alpha} \geq \varepsilon,
$$


so we obtain the conclusion of the lemma in the case (A). In the case (B) we pick $\delta=$ $(1-\varepsilon)\left(\gamma_{2}-\left(\frac{1}{\alpha}-\frac{1}{\beta}\right)\right)$. Since $\varepsilon \leq \varepsilon_{0} \leq \frac{\gamma_{2}-\left(\frac{1}{\alpha}-\frac{1}{\beta}\right)}{2(d+3) / \alpha} \leq 1 / 4$ we get $\frac{(d+3) \varepsilon}{\alpha} \leq \frac{\gamma_{2}-\left(\frac{1}{\alpha}-\frac{1}{\beta}\right)}{2}=$ $\delta / 2(1-\varepsilon)$. As in the case $(\mathrm{A})$ we obtain

$$
\delta-\frac{(d+3) \varepsilon}{\alpha} \geq \delta\left(1-\frac{1}{2(1-\varepsilon)}\right) \geq(1-2 \varepsilon) \frac{(d+3) \varepsilon}{\alpha} \geq \varepsilon .
$$

This completes the proof in the case $0<t-s \leq \tau_{0}$.

In the case $0<t-s \geq \tau_{0}$ the conclusion is trivial since

$$
\sup _{y \in \mathbb{R}^{d}}\left|G_{s-t}\left((x-y)\left(A_{s}^{T}(x)\right)^{-1}\right)-G_{s-t}\left((x-y)\left(A_{t}^{T}(x)\right)^{-1}\right)\right| \leq 2 G_{s-t}(0)
$$

and

$$
\left|\operatorname{det}\left(A_{s}^{T}(x)\right)^{-1}\right| \int_{\mathbb{R}^{d}}\left|G_{s-t}\left((x-y)\left(A_{s}^{T}(x)\right)^{-1}\right)-G_{s-t}\left((x-y)\left(A_{t}^{T}(x)\right)^{-1}\right)\right| d y \leq 2 .
$$

Lemma 5.10. Suppose that $0<s-t \leq \tau$. We have

$$
\int_{\mathbb{R}^{d}}\left|q_{t, s}^{(0)}(x, y)\right| d y \leq c(s-t)^{-1+\varepsilon}, x \in \mathbb{R}^{d} .
$$

Moreover,

$$
\left|q_{t, s}^{(0)}(x, y)\right| \leq c(s-t)^{-1+\varepsilon} G_{s-t}(0), x, y \in \mathbb{R}^{d} .
$$

The constant $c=c(\kappa(\tau))$. If $\tau \leq \tau_{0}$, then $c=c(\bar{h})$.

Proof. Let

$$
L_{t}^{z} f(x)=\sum_{k=1}^{d} \mathrm{P} . \mathrm{V} \cdot \int_{\mathbb{R}}\left[f\left(x+u e_{k} A_{t}^{T}(z)+U_{t}\left(z, u e_{k}\right)\right)-f(x)\right] \nu_{k}(d u) .
$$

Recall that

$$
\left(\frac{\partial}{\partial t}+L_{t, s}^{y}\right) p_{t, s}^{y}(w)=0 .
$$

It follows that

$$
\begin{aligned}
& \left|q_{t, s}^{(0)}(x, y)\right| \\
& =\left|\left(\frac{\partial}{\partial t}+L_{t}\right) p_{t, s}^{(0)}(\cdot, y)(x)\right| \\
& =\left|\left(\frac{\partial}{\partial t}+L_{t}^{x}\right) p_{t, s}^{y}(\cdot)(x-y)\right| \\
& =\left|\left(-L_{t, s}^{y}+L_{t}^{x}\right) p_{t, s}^{y}(\cdot)(x-y)\right| \\
& \leq\left|\sum_{k=1}^{d} \mathrm{P} \cdot \mathrm{V} \cdot \int_{|u|<R_{s-t}^{(k)}}\left[p_{t, s}^{y}\left(x-y+u e_{k} A_{t}^{T}(x)\right)-p_{t, s}^{y}\left(x-y+u e_{k} A_{s}^{T}(y)\right)\right] \nu_{k}(d u)\right| \\
& +\mid \sum_{k=1}^{d} \mathrm{P} \cdot \mathrm{V} \cdot \int_{|u|<R_{s-t}^{(k)}}\left[p_{t, s}^{y}\left(x-y+u e_{k} A_{t}^{T}(x)+U_{t}\left(x, u e_{k}\right)\right)\right. \\
& +\left|\sum_{k=1}^{d} \int_{|u| \geq R_{s-t}^{(k)}}\left[p_{t, s}^{y}\left(x-y+u e_{k} A_{t}^{T}(x)+U_{t}\left(x, u e_{k}\right)\right)-p_{t, s}^{y}(x-y)\right] \nu_{k}(d u)\right| \\
& =\mathrm{I}(x, y)+\operatorname{II}(x, y)+\operatorname{III}(x, y) .
\end{aligned}
$$


For the sake of simplicity we will denote $p(w)=p_{t, s}^{y}(w)$ and $z=x-y$. To handle the term $\mathrm{I}(x, y)$ we have to estimate

$$
\text { P.V. } \int_{|u|<R_{s-t}^{(k)}}\left[p\left(z+u e_{k} A_{t}^{T}(x)\right)-p\left(z+u e_{k} A_{s}^{T}(y)\right)\right] \nu_{k}(d u) .
$$

Because of the symmetry, we can re-write this integral as

$$
\int_{|u|<R_{s-t}^{(k)}}\left[p\left(z+u e_{k} A_{t}^{T}(x)\right)-p\left(z+u e_{k} A_{s}^{T}(y)\right)-\nabla p(z)\left(u e_{k}\left(A_{t}^{T}(x)-A_{s}^{T}(y)\right)\right)\right] \nu_{k}(d u) .
$$

We have

$$
\begin{aligned}
& p\left(z+u e_{k} A_{t}^{T}(x)\right)-p\left(z+u e_{k} A_{s}^{T}(y)\right)-\nabla p(z)\left(u e_{k}\left(A_{t}^{T}(x)-A_{s}^{T}(y)\right)\right) \\
& =\left[\nabla p\left(z+\theta u e_{k} A_{t}^{T}(x)+(1-\theta) u e_{k} A_{s}^{T}(y)\right)-\nabla p(z)\right]\left(u e_{k}\left(A_{t}^{T}(x)-A_{s}^{T}(y)\right)\right) \\
& =\left[\nabla p\left(z+u e_{k} A_{s}^{T}(y)\right)-\nabla p\left(z+\theta u e_{k} A_{t}^{T}(x)+(1-\theta) u e_{k} A_{s}^{T}(y)\right)\right]\left(u e_{k}\left(A_{t}^{T}(x)-A_{s}^{T}(y)\right)\right) \\
& +\left[\nabla p\left(z+u e_{k} A_{s}^{T}(y)\right)-\nabla p(z)\right]\left(u e_{k}\left(A_{t}^{T}(x)-A_{s}^{T}(y)\right)\right) \\
& =\Delta_{1}+\Delta_{2},
\end{aligned}
$$

where $\theta \in[0,1]$. Next,

$\nabla p\left(z+u e_{k} A_{s}^{T}(y)\right)-\nabla p\left(z+\theta u e_{k} A_{t}^{T}(x)+(1-\theta) u e_{k} A_{s}^{T}(y)\right)=\left(\theta u e_{k}\left(A_{s}^{T}(y)-A_{t}^{T}(x)\right) \nabla^{2} p(\xi)\right.$, where

$$
\left.\xi=z+\theta^{*} u e_{k} A_{t}^{T}(x)+\left(1-\theta^{*}\right) u e_{k} A_{s}^{T}(y)\right),|u| \leq R_{s-t}^{(k)} .
$$

Troughout the whole proof we pick $\delta=\frac{(1-2 \varepsilon) \gamma_{1}}{\beta}$ in the case $(\mathrm{A})$ and $\delta=(1-2 \varepsilon)((1+$ $\left.\left.\gamma_{1}\right) / \beta-1 / \alpha\right)$ in the case (B). Note that such choice of $\delta$ is dictated by Lemma 5.8, since we are going to use some arguments contained therein.

Let $|z|^{1+\gamma_{1}} \leq \frac{(s-t)^{\delta}}{\left|M_{s-t}^{-1}\right|}$, that is $y \in D(\delta, x)$. By Lemma 5.6 we have

$$
\left|\xi\left(A_{s}^{T}(y)\right)^{-1} M_{s-t}^{-1}-z\left(A_{s}^{T}(x)\right)^{-1} M_{s-t}^{-1}\right| \leq c .
$$

Applying this and (74), we arrive at

$$
\left|\nabla^{2} p(\xi)\right| \leq c \frac{\left|M_{s-t}^{-1}\right|^{2}}{(s-t)^{2 \varepsilon(1+1 / \alpha)}} e^{-\left|z\left(A_{s}^{-1}(x)\right)^{T} M_{s-t}^{-1}\right|} G_{s-t}(0) .
$$

This implies

$$
\left|\Delta_{1}\right| \leq c|u|^{2}\left|A_{s}^{T}(y)-A_{t}^{T}(x)\right|^{2} \frac{\left|M_{s-t}^{-1}\right|^{2}}{(s-t)^{2 \varepsilon(1+1 / \alpha)}} e^{-\left|z\left(A_{s}^{-1}(x)\right)^{T} M_{s-t}^{-1}\right|} G_{s-t}(0)
$$

Next, by Lemma 4.4,

$$
\int_{|u|<R_{s-t}^{(k)}} u^{2} \nu_{k}(d u) \leq\left(R_{s-t}^{(k)}\right)^{2}(s-t)^{\varepsilon-1} \leq\left|M_{s-t}\right|^{2}(s-t)^{\varepsilon-1} .
$$

Hence,

$$
\begin{aligned}
\mathrm{I}_{1}(x, y): & =\int_{|u|<R_{s-t}^{(k)}}\left|\Delta_{1}\right| \nu_{k}(d u) \\
& \leq c G_{s-t}(0)\left(\left|M_{s-t}^{-1}\right|\left|M_{s-t}\right|\right)^{2}\left|A_{s}^{T}(y)-A_{t}^{T}(x)\right|^{2} e^{-\left|z\left(A_{s}^{-1}(x)\right)^{T} M_{s-t}^{-1}\right|}(s-t)^{-\varepsilon(1+2 / \alpha)-1} .
\end{aligned}
$$

Now, let us estimate the second summand $\Delta_{2}$ in the right hand side of (90). Since $p(w)=\left|\operatorname{det}\left(\left(A_{s}^{T}(y)\right)^{-1}\right)\right| G_{s-t}\left(w\left(A_{s}^{T}(y)\right)^{-1}\right), w \in \mathbb{R}^{d}$, we have

$$
\nabla p(w)=\left|\operatorname{det}\left(\left(A_{s}^{T}(y)\right)^{-1}\right)\right| \nabla G_{s-t}\left(w\left(A_{s}^{T}(y)\right)^{-1}\right)\left(A_{s}^{T}(y)\right)^{-1} .
$$


Hence,

$$
\begin{aligned}
& \left|\nabla p\left(z+u e_{k} A_{s}^{T}(y)\right)-\nabla p(z)\right| \\
& \leq c\left|\nabla G_{s-t}\left(z\left(A_{s}^{T}(y)\right)^{-1}+u e_{k}\right)-\nabla G_{s-t}\left(z\left(A_{s}^{T}(y)\right)^{-1}\right)\right| \\
& \leq c\left|\frac{\partial}{\partial w_{k}} \nabla G_{s-t}(\xi)\right||u|
\end{aligned}
$$

where $\xi=z\left(A_{s}^{T}(y)\right)^{-1}+\theta u e_{k}, 0 \leq \theta \leq 1$. By Lemma 5.6, we obtain

$$
\left|\xi M_{s-t}^{-1}-z\left(A_{s}^{T}(x)\right)^{-1} M_{s-t}^{-1}\right| \leq c .
$$

Applying this and (74), we arrive at

$$
\left|\frac{\partial}{\partial w_{k}} \nabla G_{s-t}(\xi)\right| \leq c e^{-\left|z\left(A_{s}^{-1}(x)\right)^{T} M_{s-t}^{-1}\right|}(s-t)^{-(2+2 / \alpha) \varepsilon} \frac{1}{R_{s-t}^{(k)}}\left|M_{s-t}^{-1}\right| G_{s-t}(0) .
$$

Then we have

$$
\begin{aligned}
& \left|\Delta_{2}\right|=\left|\left[\nabla p\left(z+u e_{k} A_{s}^{T}(y)\right)-\nabla p(z)\right]\left(u e_{k}\left(A_{t}^{T}(y)-A_{t}^{T}(x)\right)\right)\right| \\
& \leq(s-t)^{-(2+2 / \alpha) \varepsilon} \frac{1}{R_{s-t}^{(k)}}\left|M_{s-t}^{-1}\right| G_{s-t}(0)|u|^{2}\left|A_{t}^{T}(x)-A_{s}^{T}(y)\right| e^{-\left|z\left(A_{s}^{-1}(x)\right)^{T} M_{s-t}^{-1}\right|} .
\end{aligned}
$$

Hence, since $\int_{|u|<R_{s-t}^{(k)}} u^{2} \nu_{k}(d u) \leq\left(R_{s-t}^{(k)}\right)^{2}(s-t)^{\varepsilon-1}$, we have

$$
\begin{aligned}
\mathrm{I}_{2}(x, y) & :=\int_{|u|<R_{s-t}^{(k)}}\left|\Delta_{2}\right| \nu_{k}(d u) \\
& \leq c G_{s-t}(0)\left|M_{s-t}^{-1}\right| R_{s-t}^{(k)}\left|A_{t}^{T}(x)-A_{s}^{T}(y)\right| e^{-\left|z\left(A_{s}^{-1}(x)\right)^{T} M_{s-t}^{-1}\right|}(s-t)^{-(2+2 / \alpha) \varepsilon}(s-t)^{\varepsilon-1} \\
& \leq c G_{s-t}(0)\left|M_{s-t}^{-1}\right|\left|M_{s-t}\right|\left|A_{t}^{T}(x)-A_{s}^{T}(y)\right| e^{-\left|z\left(A_{s}^{-1}(x)\right)^{T} M_{s-t}^{-1}\right|}(s-t)^{-(2+2 / \alpha) \varepsilon}(s-t)^{\varepsilon-1}
\end{aligned}
$$

We observe that, by (68),

$$
|x-y|^{\gamma_{1}} \leq\left(\frac{(s-t)^{\delta}}{\left|M_{s-t}^{-1}\right|}\right)^{\frac{\gamma_{1}}{1+\gamma_{1}}} \leq c(s-t)^{(\delta+(1-\varepsilon) / \beta) \frac{\gamma_{1}}{1+\gamma_{1}}} \leq c(s-t)^{((1-\varepsilon) / \beta) \frac{\gamma_{1}}{1+\gamma_{1}}}
$$

In the case $(\mathrm{A})\left|M_{s-t}^{-1}\right|\left|M_{s-t}\right|=1$, hence

$$
\begin{aligned}
\left|M_{s-t}^{-1}\right|\left|M_{s-t}\right|\left|A_{s}^{T}(y)-A_{t}^{T}(x)\right| & \leq\left|A_{s}^{T}(y)-A_{s}^{T}(x)\right|+\left|A_{s}^{T}(y)-A_{t}^{T}(y)\right| \\
& \leq c\left(|x-y|^{\gamma_{1}}+(s-t)^{\gamma_{2}}\right) \\
& \leq c\left((s-t)^{\frac{1-\varepsilon}{\beta} \frac{\gamma_{1}}{1+\gamma_{1}}}+(s-t)^{\gamma_{2}}\right) \\
& \leq c(s-t)^{(1-\varepsilon) \rho}
\end{aligned}
$$

where $\rho=\min \left\{\gamma_{1} / \beta\left(1+\gamma_{1}\right), \gamma_{2}\right\}$. In the case (B) we have, by (67) and(68),

$$
\begin{aligned}
& \left|M_{s-t}^{-1}\right|\left|M_{s-t}\right|\left|A_{s}^{T}(y)-A_{t}^{T}(x)\right| \leq\left(\left|M_{s-t}^{-1}\right|\left|M_{s-t}\right|\right)\left|A_{s}^{T}(y)-A_{s}^{T}(x)\right|+\left|A_{s}^{T}(y)-A_{t}^{T}(y)\right| \\
& \leq c\left(\left|M_{s-t}^{-1}\right|\left|M_{s-t}\right|\right)\left(|x-y|^{\gamma_{1}}+(s-t)^{\gamma_{2}}\right) \\
& \leq c\left(\left|M_{s-t}\right|\left|M_{s-t}^{-1}\right|^{\frac{1}{1+\gamma_{1}}}(s-t)^{\delta \frac{\gamma_{1}}{1+\gamma_{1}}}+\left|M_{s-t}^{-1}\right|\left|M_{s-t}\right|(s-t)^{\gamma_{2}}\right) \\
& \leq(s-t)^{(1-\varepsilon)\left(\frac{1}{\beta}-\frac{1}{\left(1+\gamma_{1}\right) \alpha}\right)+\delta \frac{\gamma_{1}}{1+\gamma_{1}}}+(s-t)^{(1-\varepsilon)\left(\frac{1}{\beta}-\frac{1}{\alpha}\right)+\gamma_{2}} \\
& \leq c(s-t)^{(1-\varepsilon) \rho} \text {, }
\end{aligned}
$$


where $\rho=\min \left\{1 / \beta-1 /\left(1+\gamma_{1}\right) \alpha, \gamma_{2}+1 / \beta-1 / \alpha\right\}$. This implies that for $y \in D(\delta, x)$,

$$
\begin{aligned}
\mathrm{I}(x, y) & \leq c \mathrm{I}_{2}(x, y) \\
& \leq c G_{s-t}(0)\left|M_{s-t}^{-1}\right|\left|M_{s-t}\right|\left|A_{t}^{T}(x)-A_{s}^{T}(y)\right| e^{-\left|z\left(A_{s}^{-1}(x)\right)^{T} M_{s-t}^{-1}\right|}(s-t)^{-(1+2 / \alpha) \varepsilon}(91) \\
& \leq c G_{s-t}(0)\left|M_{s-t}^{-1}\right|\left|M_{s-t}\right|\left|A_{t}^{T}(x)-A_{s}^{T}(y)\right|(s-t)^{-(1+2 / \alpha) \varepsilon-1} e^{-\frac{|z|}{\|A\||| M_{s-t} \mid}} \\
& \leq c G_{s-t}(0)(s-t)^{\rho(1-\varepsilon)-(2+2 / \alpha) \varepsilon}(s-t)^{\varepsilon-1} e^{-\frac{|z|}{|| A|| M_{s-t}}} \\
& \leq c G_{s-t}(0)(s-t)^{\varepsilon-1} e^{-\frac{|z|}{\| A|| M_{s-t} \mid}}
\end{aligned}
$$

provided

$$
\varepsilon \leq \frac{\rho(1-\varepsilon)}{2+2 / \alpha}
$$

That is, when

$$
0<\varepsilon \leq \frac{\min \left\{\gamma_{1} / \beta\left(1+\gamma_{1}\right), \gamma_{2}\right\}}{2+2 / \alpha+\min \left\{\gamma_{1} / \beta\left(1+\gamma_{1}\right), \gamma_{2}\right\}} \quad \text { in the case (A) }
$$

and

$$
0<\varepsilon \leq \frac{\min \left\{1 / \beta-1 /\left(1+\gamma_{1}\right) \alpha, \gamma_{2}+1 / \beta-1 / \alpha\right\}}{2+2 / \alpha+\min \left\{1 / \beta-1 /\left(1+\gamma_{1}\right) \alpha, \gamma_{2}+1 / \beta-1 / \alpha\right\}} \quad \text { in the case (B). }
$$

Hence (93) holds for $\varepsilon \leq \varepsilon_{0}$. Next,

$$
\left|A_{s}^{T}(y)-A_{t}^{T}(x)\right| \leq\left|A_{s}^{T}(y)-A_{s}^{T}(x)\right|+\left|A_{s}^{T}(y)-A_{t}^{T}(y)\right| \leq c\left(|x-y|^{\gamma_{1}}+(s-t)^{\gamma_{2}}\right) .
$$

By (91), Lemma 5.5 used twice ( with $\rho=\gamma_{1}$ or with $\rho=0$ ), (80) and finally (68), we arrive at

$$
\begin{aligned}
& \int_{D(\delta, x)}|I(x, y)| d y \\
\leq & c G_{s-t}(0) \operatorname{det}\left(M_{s-t}\right)\left|M_{s-t}^{-1}\right|\left|M_{s-t}\right|\left(\left|M_{s-t}\right|^{\gamma_{1}}+(s-t)^{\gamma_{2}}\right)(s-t)^{-\varepsilon(1+2 / \alpha)-1} \\
\leq & c(t-s)^{-\varepsilon(2+(d+2) / \alpha)}\left|M_{s-t}^{-1}\right|\left|M_{s-t}\right|\left(\left|M_{s-t}\right|^{\gamma_{1}}+(s-t)^{\gamma_{2}}\right)(s-t)^{\varepsilon-1} \\
\leq & c(t-s)^{-\varepsilon(2+(d+2) / \alpha)}\left|M_{s-t}^{-1}\right|\left|M_{s-t}\right|\left((s-t)^{(1-\varepsilon) \gamma_{1} / \beta}+(s-t)^{\gamma_{2}}\right)(s-t)^{\varepsilon-1} .
\end{aligned}
$$

In the case (A) we have $\left|M_{s-t}^{-1}\right|\left|M_{s-t}\right|=1$, hence

$$
\int_{D(\delta, x)}|I(x, y)| d y \leq c(s-t)^{-1+\varepsilon},
$$

if $\varepsilon \leq \min \left\{\frac{\gamma_{1}}{\gamma_{1}+\beta(2+(d+2) / \alpha)}, \frac{\gamma_{2}}{2+(d+2) / \alpha}\right\}$, which is satisfied with our assumptions on $\varepsilon$. In the case (B) we have, by (67) and (68),$\left|M_{s-t}^{-1}\right|\left|M_{s-t}\right| \leq c(s-t)^{(1-\varepsilon)(-1 / \alpha+1 / \beta)}$, so

$$
\begin{aligned}
\int_{D(\delta, x)}|I(x, y)| d y & \leq c\left[(t-s)^{-\varepsilon(2+(d+2) / \alpha)+(1-\varepsilon)\left(\left(1+\gamma_{1}\right) / \beta-1 / \alpha\right)}\right. \\
& \left.+(t-s)^{-\varepsilon(2+(d+2) / \alpha)+(1-\varepsilon)(1 / \beta-1 / \alpha)+\gamma_{2}}\right](s-t)^{-1+\varepsilon} \\
& \leq c(s-t)^{-1+\varepsilon} .
\end{aligned}
$$

provided $-\varepsilon(2+(d+2) / \alpha)+(1-\varepsilon)\left(\left(1+\gamma_{1}\right) / \beta-1 / \alpha\right) \geq 0$ and $-\varepsilon(2+(d+2) / \alpha)+(1-\varepsilon)(1 / \beta-1 / \alpha)+\gamma_{2} \geq 0$. That is

$$
\varepsilon \leq \min \left\{\frac{\left(1+\gamma_{1}\right) / \beta-1 / \alpha}{\left(\gamma_{1}+1\right) / \beta+2+(d+1) / \alpha}, \frac{\left(1 / \beta-1 / \alpha+\gamma_{2}\right)}{1 / \beta+2+(d+1) / \alpha}\right\} .
$$

Again, this is true with our assumptions. 
Now we deal with the estimates of $\mathrm{I}(x, y)$ over $D^{c}(\delta, x)$. We note that our assumptions yield that in the case (A) $\varepsilon \leq \frac{\gamma_{1} \alpha}{2(d+3) \beta}$, while in the case (B) $\varepsilon \leq \frac{\left(1+\gamma_{1}\right) / \beta-1 / \alpha}{2(d+3) / \alpha}$. We have

$$
\left|p\left(z+u e_{k} A_{t}^{T}(x)\right)-p\left(z+u e_{k} A_{s}^{T}(y)\right)-\nabla p(z)\left(u e_{k}\left(A_{t}^{T}(y)-A_{t}^{T}(x)\right)\right)\right| \leq c\left|\nabla^{2} p(\xi)\right| u^{2}
$$

for

$$
\xi=z+\lambda\left(\theta u e_{k} A_{t}^{T}(x)+(1-\theta) u e_{k} A_{s}^{T}(y)\right),|u| \leq R_{s-t}^{(k)},
$$

with $\lambda, \theta \in[0,1]$. We note that

$$
\begin{aligned}
|\xi-z| & \leq \lambda\left(\theta|u|\left|e_{k} A_{t}^{T}(x)\right|+(1-\theta)|u|\left|e_{k} A_{t}^{T}(y)\right|\right) \\
& \leq|u|\|A\| \\
& \leq R_{s-t}^{(k)}\|A\| \\
& \leq\left|M_{s-t}\right|\|A\| .
\end{aligned}
$$

Hence, by (75) and then by (67), we get

$$
\begin{aligned}
\left|\nabla^{2} p(\xi)\right| & \leq c\left|M_{s-t}^{-1}\right|^{2} \exp \left(-\frac{|z|}{|| A||\left|M_{s-t}\right|}\right)(s-t)^{-(2+\varepsilon) / \alpha} G_{s-t}(0) \\
& \leq c \exp \left(-\frac{|z|}{|| A||\left|M_{s-t}\right|}\right)(s-t)^{-4 / \alpha} G_{s-t}(0) .
\end{aligned}
$$

Combined with (96) it yields

$$
\begin{aligned}
\mathrm{I}(x, y) & \leq c \exp \left(-\frac{|z|}{|| A||\left|M_{s-t}\right|}\right)(s-t)^{-4 / \alpha} G_{s-t}(0) \sum_{k=1}^{d} \int_{|u|<R_{s-t}^{(k)}}|u|^{2} \nu_{k}(d u) d u \\
& \leq c \exp \left(-\frac{|z|}{\| A||\left|M_{s-t}\right|}\right)(s-t)^{-\frac{d+4}{\alpha}},
\end{aligned}
$$

since $G_{s-t}(0) \leq c(s-t)^{-\frac{d}{\alpha}}$. Next, observe that $\frac{|z|}{\| A||\left|M_{s-t}\right|} \geq c(s-t)^{-\frac{\varepsilon \gamma_{1}}{\beta}}$ for $y \in D^{c}(\delta, x)$, which implies that

Using this we obtain

$$
\mathrm{I}(x, y) \leq c \exp \left(-\frac{|z|}{2|| A||\left|M_{s-t}\right|}\right) .
$$

$$
\int_{D^{c}(\delta, x)} \mathrm{I}(x, y) d y \leq c .
$$

Now we estimate $\operatorname{II}(x, y)$. We have

$$
\left|p\left(z+u e_{k} A_{t}^{T}(x)+U_{t}\left(x, u e_{k}\right)\right)-p\left(z+u e_{k} A_{t}^{T}(x)\right)\right| \leq\left|\nabla p(\xi) U_{t}\left(x, u e_{k}\right)\right|,
$$

where

with $\lambda \in(0,1)$.

$$
\xi=z+u e_{k} A_{t}^{T}(x)+\lambda U_{t}\left(x, u e_{k}\right),|u| \leq R_{s-t}^{(k)},
$$

Let $|z|^{1+\gamma_{1}} \leq \frac{(s-t)^{\delta}}{\left|M_{s-t}^{-1}\right|}$. By Lemma 5.6, we have

$$
\left|\xi\left(A_{s}^{T}(y)\right)^{-1} M_{s-t}^{-1}-z\left(A_{s}^{T}(x)\right)^{-1} M_{s-t}^{-1}\right| \leq c .
$$

Applying this and (73), we arrive at

$$
|\nabla p(\xi)| \leq c \frac{\left|M_{s-t}^{-1}\right|}{(s-t)^{\varepsilon(1+1 / \alpha)}} e^{-\left|z\left(A_{s}^{-1}(x)\right)^{T} M_{s-t}^{-1}\right|} G_{s-t}(0) .
$$

From Lemma 4.4 we infer that $\int_{|u|<R_{s-t}^{(k)}}|u|^{\gamma_{3}} \nu_{k}(d u) \leq c\left(R_{s-t}^{(k)}\right)^{\gamma_{3}}(s-t)^{\varepsilon-1}$. This combined with (99) yield

$$
\mathrm{II}(x, y) \leq c \frac{\left|M_{s-t}^{-1}\right|\left|M_{s-t}\right|^{\gamma_{3}}}{(s-t)^{\varepsilon(1+1 / \alpha)}} e^{-\left|z\left(A_{s}^{-1}(x)\right)^{T} M_{s-t}^{-1}\right|} G_{s-t}(0)(s-t)^{\varepsilon-1} .
$$


We also note that

$$
\mathrm{II}(x, y) \leq c G_{s-t}(0)(s-t)^{\varepsilon-1} e^{-\frac{|z|}{\|A\||| M_{s-t} \mid}} .
$$

To prove it in the case (A), using (67), we observe that

$$
\left|M_{s-t}^{-1}\right|\left|M_{s-t}\right|^{\gamma_{3}}=\left|M_{s-t}\right|^{\gamma_{3}-1} \leq c(s-t)^{(1-\varepsilon)\left(\gamma_{3}-1\right) / \beta} .
$$

Hence, from (101) we obtain (102) provided

$$
\varepsilon \leq \frac{\gamma_{3}-1}{\gamma_{3}+\beta(1+1 / \alpha)},
$$

which holds with our assumptions. To prove it in the case (B) we observe that, by (67) and (68),

$$
\left|M_{s-t}^{-1}\right|\left|M_{s-t}\right|^{\gamma_{3}} \leq c(s-t)^{(1-\varepsilon)\left(\gamma_{3} / \beta-1 / \alpha\right)} .
$$

Hence, from (101) we obtain (102) provided

$$
\varepsilon \leq \frac{\gamma_{3}-\beta / \alpha}{\gamma_{3}+\beta}
$$

which again holds in this case.

Applying Lemma 5.5 (with $\rho=0$ ), the estimate (101), (80) and finally (68), we obtain

$$
\begin{aligned}
\int_{D(\delta, x)}|\operatorname{II}(x, y)| d y & \leq c G_{s-t}(0) \operatorname{det}\left(M_{s-t}\right)\left|M_{s-t}^{-1}\right|\left|M_{s-t}\right|^{\gamma_{3}}(s-t)^{-(1+1 / \alpha) \varepsilon}(s-t)^{\varepsilon-1} \\
& \leq c(t-s)^{-\varepsilon(1+(d+1) / \alpha)}\left|M_{s-t}^{-1}\right|\left|M_{s-t}\right|^{\gamma_{3}}(s-t)^{\varepsilon-1} \\
& \leq c(t-s)^{-\varepsilon(1+(d+1) / \alpha)}\left|M_{s-t}^{-1}\right|\left|M_{s-t}\right|(s-t)^{(1-\varepsilon)\left(\gamma_{3}-1\right) / \beta}(s-t)^{\varepsilon-1} .
\end{aligned}
$$

Then, by the same arguments as we applied to handle the term $\mathrm{I}(x, y)$, we obtain

$$
\int_{D(\delta, x)}|\mathrm{II}(x, y)| d y \leq c(s-t)^{-1+\varepsilon}
$$

in both cases: (A) (since $\varepsilon \leq \frac{\gamma_{3}-1}{\gamma_{3}-1+\beta(1+(d+1) / \alpha)}$ ) and (B) (since $\varepsilon \leq \frac{\gamma_{3}-\beta / \alpha}{\gamma_{3}+\beta(1+d / \alpha)}$ ). Moreover, again the same reasoning, as when $\mathrm{I}(x, y)$ was explored, leads to

$$
\int_{D^{c}(\delta, x)}|\mathrm{II}(x, y)| d y \leq c
$$

and

By Lemma 5.8 ,

$$
\mathrm{II}(x, y) \leq c \exp \left(-\frac{|z|}{2|| A||\left|M_{s-t}\right|}\right), y \in D^{c}(\delta, x) .
$$

$$
\begin{aligned}
\int_{\mathbb{R}^{d}} \operatorname{III}(x, y) d y & \leq c \sum_{k=1}^{d} \int_{|u| \geq R_{s-t}^{(k)}} \nu_{k}(d u) \leq c \sum_{k=1}^{d} h_{k}\left(R_{s-t}^{(k)}\right) \\
& =c \sum_{k=1}^{d} h_{k}\left(h_{k}^{-1}\left((s-t)^{-1+\varepsilon}\right)\right)=c d(s-t)^{-1+\varepsilon} .
\end{aligned}
$$

Using this, (94), (95), (98), (103) and (104) we get the first assertion of the lemma.

Finally it is clear that

$$
\operatorname{III}(x, y) \leq c G_{s-t}(0) \sum_{k=1}^{d} \int_{|u| \geq R_{s-t}^{(k)}} \nu_{k}(d u) \leq c G_{s-t}(0)(s-t)^{-1+\varepsilon} .
$$

This together with (92), (102) and (105) prove the second assertion of the lemma. Finally, we remark that all the constants appearing in the above estimates $c$ depned on $\tau$ through $\kappa(\tau)$ and for $\tau \leq \tau_{0}$ the constants $c=c(\bar{h})$. 
Lemma 5.11. Assume (I) and let $\tau>0$. For $0<t<s \leq \tau$ and $y \in \mathbb{R}^{d}$ we have

$$
\int_{\mathbb{R}^{d}}\left|q_{t, s}^{(0)}(x, y)\right| d x \leq c(s-t)^{-1+\varepsilon},
$$

where $c=c\left(C_{8}, \kappa(\tau)\right)$. If $\tau \leq \tau_{0}$, then $c=c\left(C_{8}, \bar{h}\right)$.

Proof. The proof repeats partially the proof of Lemma 5.10. Namely, take the decomposition

$$
q_{t, s}^{(0)}(x, y)=\mathrm{I}(x, y)+\mathrm{II}(x, y)+\mathrm{III}(x, y)
$$

from this proof, and observe that literally the same estimates as in the above proof yield the required intergal-in- $x$ bound for the first two terms:

$$
\int_{\mathbb{R}^{d}}(|\mathrm{I}(x, y)|+|\mathrm{II}(x, y)|) d x \leq c(s-t)^{-1+\varepsilon} .
$$

For the third term, we have to use the additional assumption (I). Namely,

$$
\begin{aligned}
\|\operatorname{III}(\cdot, y)\|_{L_{1}} & \leq \sum_{k=1}^{d} \int_{|u| \geq R_{s-t}^{(k)}} \int_{\mathbb{R}^{d}}\left[p_{t, s}^{y}\left(x-y+u e_{k} A_{t}^{T}(x)+U_{t}\left(x, u e_{k}\right)\right)+p_{t, s}^{y}(x-y)\right] \nu_{k}(d u) \\
& =\int_{z: z_{k} \geq R_{s-t}^{(k)}, k=1, \ldots, d}\left[\left\|T^{t, z} p_{t, s}^{y}(\cdot-y)\right\|_{L_{1}}+\left\|p_{t, s}^{y}(\cdot-y)\right\|_{L_{1}}\right] \mu(d z) \\
& \leq c \int_{z: z_{k} \geq R_{s-t}^{(k)}, k=1, \ldots, d} \mu(d z) \\
& \leq c(s-t)^{-1+\varepsilon},
\end{aligned}
$$

where in the penultimate inequality we have used (I) and the identity

$$
\int_{\mathbb{R}^{d}} p_{t, s}^{y}(x, y) d x=1,
$$

which is easy to derive from the definition of $p_{t, s}^{y}(x, y)$.

Lemma 5.12. Fix $\tau>0$. For any $\xi \in(0,1], \zeta>0,0<s-t \leq \tau, x, y \in \mathbb{R}^{d}$, if $s-t \geq \xi$, then we have

$$
\begin{aligned}
& \left|\sum_{k=1}^{d} \int_{|u|<R_{s-t}^{(k)} \wedge \zeta}\left[p_{t, s}^{y}\left(x-y+u e_{k} A_{t}^{T}(x)\right)-p_{t, s}^{y}\left(x-y+u e_{k} A_{s}^{T}(y)\right)\right] \nu_{k}(d u)\right| \\
+ & \left|\sum_{k=1}^{d} \int_{|u|<R_{s-t}^{(k)} \wedge \zeta}\left[p_{t, s}^{y}\left(x-y+u e_{k} A_{t}^{T}(x)+U_{t}\left(x, u e_{k}\right)\right)-p_{t, s}^{y}\left(x-y+u e_{k} A_{t}^{T}(x)\right)\right] \nu_{k}(d u)\right| \\
\leq & c \sum_{k=1}^{d} \int_{|u|<R_{s-t}^{(k)} \wedge \zeta}\left(|u|^{2}+|u|^{\gamma_{3}}\right) \nu_{k}(d u),
\end{aligned}
$$

where $c=c(\xi, \kappa(\tau))$.

Proof. The lemma follows from the estimates of $\Delta_{1}, \Delta_{2}$ (in the proof of Lemma [5.10), (99) and (100).

Lemma 5.13. Fix $\tau>0$. We have

$$
\lim _{r \rightarrow \infty} \sup _{x \in \mathbb{R}^{d}, 0<s-t<\tau} \int_{B^{c}(x, r)}\left|q_{t, s}^{(0)}(x, y)\right| d y=0
$$

and

$$
\lim _{r \rightarrow \infty} \sup _{x \in \mathbb{R}^{d}, 0<s-t<\tau} \int_{B^{c}(x, r)}\left|p_{t, s}^{(0)}(x, y)\right| d y=0 .
$$


Proof. Keeping the notation from Lemma 5.10 we have

$$
\left|q_{t, s}^{(0)}(x, y)\right| \leq \mathrm{I}(x, y)+\mathrm{II}(x, y)+\operatorname{III}(x, y) .
$$

By (92), (97) and (102), (105) we get for any $x, y \in \mathbb{R}^{d}$,

$$
\mathrm{I}(x, y)+\mathrm{II}(x, y) \leq c \exp \left(-\frac{|x-y|}{2\|A\|\left|M_{s-t}\right|}\right)(s-t)^{-d / \alpha+\varepsilon-1} .
$$

By (8) and (13), for any $x \in \mathbb{R}^{d}, u \in \mathbb{R}, t>0$ we have

$$
\max _{1 \leq k \leq d}\left|u e_{k} A_{t}^{T}(x)+U_{t}\left(x, u e_{k}\right)\right| \leq\left(d C_{3}+C_{7}\right)\left(|u|^{\gamma_{3}} \vee|u|\right) .
$$

Put $r_{0}=2\left(d C_{3}+C_{7}\right)$. We bound $\operatorname{III}(x, y)$ from above by

$$
\begin{aligned}
& \left|\sum_{k=1}^{d} \int_{\frac{|x-y|^{1 / \gamma_{3}}}{r_{0}^{1 / \gamma_{3}}} \geq|u| \geq R_{s-t}^{(k)}}\left[p_{t, s}^{y}\left(x-y+u e_{k} A_{t}^{T}(x)+U_{t}\left(x, u e_{k}\right)\right)-p_{t, s}^{y}(x-y)\right] \nu_{k}(d u)\right| \\
& +\left|\sum_{k=1}^{d} \int_{|u| \geq \max \left(R_{s-t}^{(k)}, \frac{|x-y|^{1 / \gamma_{3}}}{r_{0}^{1 / \gamma_{3}}}\right)}\left[p_{t, s}^{y}\left(x-y+u e_{k} A_{t}^{T}(x)+U_{t}\left(x, u e_{k}\right)\right)-p_{t, s}^{y}(x-y)\right] \nu_{k}(d u)\right| \\
& =\operatorname{IV}(x, y)+\mathrm{V}(x, y) .
\end{aligned}
$$

Assume now that $|x-y| \geq r_{0}$. When $u$ satisfies $|x-y|^{1 / \gamma_{3}} / r_{0}^{1 / \gamma_{3}} \geq|u| \geq R_{s-t}^{(k)}$ then, by (109), we have

$$
\begin{aligned}
\left|x-y+u e_{k} A_{t}^{T}(x)+U_{t}\left(x, u e_{k}\right)\right| & \geq|x-y|-\left|u e_{k} A_{t}^{T}(x)+U_{t}\left(x, u e_{k}\right)\right| \\
& \geq|x-y|-\left(r_{0} / 2\right)\left(|u|^{\gamma_{3}} \vee|u|\right) \\
& \geq|x-y|-\left(r_{0} / 2\right) \frac{|x-y|}{r_{0}} \\
& =\frac{|x-y|}{2} .
\end{aligned}
$$

Using this and Corollary 5.4 we get

$$
\left|p_{t, s}^{y}\left(x-y+u e_{k} A_{t}^{T}(x)+U_{t}\left(x, u e_{k}\right)\right)-p_{t, s}^{y}(x-y)\right| \leq c G_{s-t}(0) \exp \left(-\frac{|x-y|}{2\|A\|\left|M_{s-t}\right|}\right) .
$$

It follows that for $|x-y| \geq r_{0}$ we have

$$
\begin{aligned}
\operatorname{IV}(x, y) & \leq c G_{s-t}(0)\left(\sum_{k=1}^{d} \int_{|u| \geq R_{s-t}^{(k)}} \nu_{k}(d u)\right) \exp \left(-\frac{|x-y|}{2\|A\|\left|M_{s-t}\right|}\right) \\
& \leq c G_{s-t}(0) \exp \left(-\frac{|x-y|}{2\|A\|\left|M_{s-t}\right|}\right),
\end{aligned}
$$

since for any $k \in\{1, \ldots, d\}$ we have $\int_{|u| \geq R_{s-t}^{(k)}} \nu_{k}(d u) \leq h_{k}\left(R_{s-t}^{(k)}\right)=\frac{1}{(s-t)^{\varepsilon-1}} \leq c$.

By elementary arguments for any $a, r>0$ we have

$$
\int_{B^{c}(x, r)} e^{-a|x-y|} d y=\frac{c}{a^{d}} \int_{a r}^{\infty} e^{-v} v^{d-1} d v \leq \frac{c}{a^{d}} e^{-a r / 2},
$$

where $c$ depends only on $d$. Using this, (108), (110) and and (68) we get for $r \geq r_{0}$

$$
\int_{B^{c}(x, r)}(\mathrm{I}(x, y)+\mathrm{II}(x, y)+\mathrm{IV}(x, y)) d y \leq c(s-t)^{-d / \alpha+\varepsilon-1}\left|M_{s-t}\right|^{d} \exp \left(\frac{-r}{4\left|M_{s-t}\right|\|A\|}\right) \leq c e^{-c_{1} r} \text {. }
$$


By Lemma 5.8, we have

$$
\begin{aligned}
& \int_{\mathbb{R}^{d}} \operatorname{IV}(x, y) d y \leq c \sum_{k=1}^{d} \int_{|u| \geq \max \left(R_{s-t}^{(k)}, r^{\left.1 / \gamma_{3} / r_{0}^{1 / \gamma_{3}}\right)}\right.} \nu_{k}(d u) \\
& \leq c \sum_{k=1}^{d} h_{k}\left(\frac{r^{1 / \gamma_{3}}}{r_{0}^{1 / \gamma_{3}}}\right) \text {. }
\end{aligned}
$$

Since $\lim _{r \rightarrow \infty} h_{k}(r)=0$, the first assertion of the lemma follows from (111) and (112).

The proof of the second follows easily from (72).

Put

$$
W=\{(t, s): t, s \in[0, \infty), t<s\} .
$$

Lemma 5.14. The function $W \times \mathbb{R}^{d} \times \mathbb{R}^{d} \ni(t, s, x, y) \rightarrow p_{t, s}^{y}(x)$ is continuous as well as the function $W \times \mathbb{R}^{d} \times \mathbb{R}^{d} \ni(t, s, x, y) \rightarrow q_{t, s}^{(0)}(x, y)$.

Proof. The first assertion follows from Lemma 4.7 and continuity of the map $(s, x) \mapsto$ $A_{s}(x)$. Recall that $q_{t, s}^{(0)}(x, y)$ is equal to

$$
\begin{aligned}
& \sum_{k=1}^{d} \int_{|u|<R_{s-t}^{(k)}}\left[p_{t, s}^{y}\left(x-y+u e_{k} A_{t}^{T}(x)\right)-p_{t, s}^{y}\left(x-y+u e_{k} A_{s}^{T}(y)\right)\right] \nu_{k}(d u) \\
& +\sum_{k=1}^{d} \int_{|u|<R_{s-t}^{(k)}}\left[p_{t, s}^{y}\left(x-y+u e_{k} A_{t}^{T}(x)+U_{t}\left(x, u e_{k}\right)\right)-p_{t, s}^{y}\left(x-y+u e_{k} A_{s}^{T}(x)\right)\right] \nu_{k}(d u) \\
& +\sum_{k=1}^{d} \int_{|u| \geq R_{s-t}^{(k)}}\left[p_{t, s}^{y}\left(x-y+u e_{k} A_{t}^{T}(x)+U_{t}\left(x, u e_{k}\right)\right)-p_{t, s}^{y}(x-y)\right] \nu_{k}(d u)
\end{aligned}
$$

Hence, the second assertion of the lemma follows from the first, Lemma 5.12, (72) and the bounded convergence theorem.

Lemma 5.15. For any $f \in C_{\infty}\left(\mathbb{R}^{d}\right), t_{0} \geq 0$ we have

$$
\lim _{W \ni(t, s) \rightarrow\left(t_{0}, t_{0}\right)}\left\|P_{t, s}^{(0)} f-f\right\|_{\infty}=0 .
$$

Proof. Note that for any $x \in \mathbb{R}^{d}, 0<t<s<\infty$ we have $\int_{\mathbb{R}^{d}} p_{t, s}^{x}(x-y) d y=1$. Using this, (83) and (72) we easily obtain the assertion of the lemma.

Lemma 5.16. For any $f \in C_{\infty}\left(\mathbb{R}^{d}\right), 0 \leq t_{0}<s_{0}$ we have

$$
\lim _{W \ni(t, s) \rightarrow\left(t_{0}, s_{0}\right)}\left\|Q_{t, s}^{(0)} f-Q_{t_{0}, s_{0}}^{(0)} f\right\|_{\infty}=0
$$

and

$$
\lim _{W \ni(t, s) \rightarrow\left(t_{0}, s_{0}\right)}\left\|P_{t, s}^{(0)} f-P_{t_{0}, s_{0}}^{(0)} f\right\|_{\infty}=0 .
$$

Proof. We give a detailed proof of the first statement and only a sketch for the second.

By Lemma 5.13 , it is enough to prove the lemma for $f$ with compact support. We note that the function $\left(W \times \mathbb{R}^{d}\right) \ni(t, s, x) \rightarrow Q_{t, s}^{(0)} f(x)$ is continuous. This follows from (89)), Lemma 5.14 and the bounded convergence theorem. Let $r>0$. Hence it is uniformly continuous on

$$
\left\{t, s, x: t \geq 0 ;|x| \leq r ;\left|t-t_{0}\right|,\left|s-s_{0}\right| \leq\left|s_{0}-t_{0}\right| / 3\right\} .
$$

It follows that

$$
\lim _{W \ni(t, s) \rightarrow\left(t_{0}, s_{0}\right)} \sup _{|x| \leq r}\left|Q_{t, s}^{(0)} f(x)-Q_{t_{0}, s_{0}}^{(0)} f(x)\right|=0 .
$$


Let $r$ be so large that the support of $f$ is contained in $B(0, r / 2)$. Next, we have

$$
\sup _{|x| \geq r, 0<s-t<2\left(s_{0}-t_{0}\right)}\left|Q_{t, s}^{(0)} f(x)\right| \leq\|f\|_{\infty} \sup _{x \in \mathbb{R}^{d}, 0<s-t<2\left(s_{0}-t_{0}\right)} \int_{B^{c}(x, r / 2)}\left|q_{t, s}^{(0)}(x, y)\right| d y .
$$

Hence

$$
\limsup _{W \ni(t, s) \rightarrow\left(t_{0}, s_{0}\right)}\left\|Q_{t, s}^{(0)} f-Q_{t_{0}, s_{0}}^{(0)} f\right\|_{\infty} \leq 2\|f\|_{\infty} \sup _{x \in \mathbb{R}^{d}, 0<s-t<2\left(s_{0}-t_{0}\right)} \int_{B^{c}(x, r / 2)}\left|q_{t, s}^{(0)}(x, y)\right| d y,
$$

which converges to 0 , if $r \rightarrow \infty$, by Lemma 5.13. This completes the proof of the first assertion.

Finally, we remark that the function $W \times \mathbb{R}^{d} \ni(t, s, x) \rightarrow P_{t, s}^{(0)} f(x)$ is continuous, due to Lemma 5.14. Next, similarly as above, we apply (107) to complete the proof of the second assertion.

Lemma 5.17. For any $0<s-t \leq \tau$ and $x, y \in \mathbb{R}^{d}$ such that $|x-y| \leq(s-t)^{1 / \alpha}$ we have

$$
\int_{\mathbb{R}^{d}}\left|p_{t, s}^{z}(x-z)-p_{t, s}^{z}(y-z)\right| d z \leq c|x-y|(s-t)^{-1 / \alpha-(d+2) \varepsilon / \alpha},
$$

where $c=c(\kappa(\tau))$.

Proof. We have

$$
\begin{aligned}
& p_{t, s}^{z}(x-z)-p_{t, s}^{z}(y-z) \\
& =\frac{1}{\left|\operatorname{det}\left(A_{s}(z)\right)\right|}\left[G_{s-t}\left((x-z)\left(A_{s}^{-1}(z)\right)^{T}\right)-G_{s-t}\left((y-z)\left(A_{s}^{-1}(z)\right)^{T}\right)\right] \\
& =\frac{1}{\left|\operatorname{det}\left(A_{s}(z)\right)\right|} \nabla G_{s-t}(\xi)\left[(x-y)\left(A_{s}^{-1}(z)\right)^{T}\right],
\end{aligned}
$$

where $\xi=(\theta(x-z)+(1-\theta)(y-z))\left(A_{s}^{-1}(z)\right)^{T}, 0 \leq \theta \leq 1$. By Lemma 5.2 and then Lemma 4.2, we obtain

$$
\begin{aligned}
\left|p_{t, s}^{z}(x-z)-p_{t, s}^{z}(y-z)\right| & \leq c|x-y| G_{s-t}(0) \frac{1}{h_{\min }^{-1}(1 /(s-t))} \frac{1}{(s-t)^{\varepsilon}} e^{-\left|\xi M_{s-t}^{-1}\right|} \\
& \leq c|x-y| G_{s-t}(0) \frac{1}{(s-t)^{\varepsilon+1 / \alpha}} e^{-\left|\xi M_{s-t}^{-1}\right|}
\end{aligned}
$$

We have

$$
\left|\xi M_{s-t}^{-1}\right| \geq\left|(x-z)\left(A_{s}^{-1}(z)\right)^{T} M_{s-t}^{-1}\right|-\left|\xi M_{s-t}^{-1}-(x-z)\left(A_{s}^{-1}(z)\right)^{T} M_{s-t}^{-1}\right| .
$$

Since $|x-y| \leq(s-t)^{1 / \alpha}$, using (68), we get

$$
\begin{aligned}
\left|\xi M_{s-t}^{-1}-(x-z)\left(A_{s}^{-1}(z)\right)^{T} M_{s-t}^{-1}\right| & \leq\left|(-(1-\theta) x+(1-\theta) y)\left(A_{s}^{-1}(z)\right)^{T} M_{s-t}^{-1}\right| \\
& \leq|x-y|\|A\|\left|M_{s-t}^{-1}\right| \\
& \leq c|x-y|(s-t)^{-1 / \alpha+\varepsilon / \alpha} \\
& \leq c .
\end{aligned}
$$

We pick $\delta>0$ in the same way as in Lemma 5.8. By the same arguments as in the proof of Lemma 5.7 for $z \in D(\delta, x)$ we have

$$
\left|(x-z)\left(A_{s}^{-1}(z)\right)^{T}-(x-z)\left(A_{s}^{-1}(x)\right)^{T}\right| \leq\|A\||x-z|^{1+\gamma_{1}} \leq(s-t)^{\delta}\|A\| \frac{1}{\left|M_{s-t}^{-1}\right|} .
$$

Hence

$\left|(x-z)\left(A_{s}^{-1}(z)\right)^{T} M_{s-t}^{-1}-(x-z)\left(A_{s}^{-1}(x)\right)^{T} M_{s-t}^{-1}\right| \leq\left.(s-t)^{\delta}\left|M_{s-t}^{-1}\right|\|A\||| M_{s-t}^{-1}\right|^{-1}=(s-t)^{\delta}\|A\|$. 
Therefore for $z \in D(\delta, x)$ we have

$$
\left|p_{t, s}^{z}(x-z)-p_{t, s}^{z}(y-z)\right| \leq c|x-y| G_{s-t}(0) \frac{1}{(s-t)^{\varepsilon+1 / \alpha}} e^{-\left|(x-z)\left(A_{s}^{-1}(x)\right)^{T} M_{s-t}^{-1}\right|} .
$$

Using the above estimates, Lemma 5.5 and Lemma 5.2 we get

$$
\begin{aligned}
& \int_{D(\delta, x)}\left|p_{t, s}^{z}(x-z)-p_{t, s}^{z}(y-z)\right| d z \\
& \leq c|x-y| G_{s-t}(0) \frac{1}{(s-t)^{\varepsilon+1 / \alpha}} \int_{D(\delta, x)} e^{-\left|(x-z)\left(A_{s}^{-1}(x)\right)^{T} M_{s-t}^{-1}\right|} d z \\
& \leq c|x-y| G_{s-t}(0) \frac{1}{(s-t)^{\varepsilon+1 / \alpha}} \operatorname{det}\left(M_{s-t}\right) \\
& \leq \frac{c|x-y|}{(s-t)^{1 / \alpha+\varepsilon}} \prod_{i=1}^{d} \frac{h_{i}^{-1}\left(1 /(s-t)^{1-\varepsilon}\right)}{h_{i}^{-1}(1 /(s-t))} .
\end{aligned}
$$

By Corollary 4.3 this is bounded from above by

$$
c|x-y|(s-t)^{-1 / \alpha-(d+2) \varepsilon / \alpha} .
$$

For $z \in D^{c}(\delta, x)$ we have

$$
\begin{aligned}
& \left|p_{t, s}^{z}(x-z)-p_{t, s}^{z}(y-z)\right| \\
& \leq c|x-y| G_{s-t}(0) \frac{1}{(s-t)^{\varepsilon+1 / \alpha}} e^{-\left|(x-z)\left(A_{s}^{-1}(z)\right)^{T} M_{s-t}^{-1}\right|} \\
& \leq c|x-y| G_{s-t}(0) \frac{1}{(s-t)^{\varepsilon+1 / \alpha}} e^{-\frac{|x-z|}{\|A\|\left|M_{s-t}\right|}} .
\end{aligned}
$$

Using (866) we infer that there exists $c$ such that

$$
\begin{aligned}
& \int_{D^{c}(\delta, x)}\left|p_{t, s}^{z}(x-z)-p_{t, s}^{z}(y-z)\right| d z \\
& \leq c|x-y| G_{s-t}(0) \frac{1}{(s-t)^{\varepsilon+1 / \alpha}} \int_{D^{c}(\delta, x)} e^{-\frac{|x-z|}{\|A\||| M_{s-t}}} d z \\
& \leq c|x-y|,
\end{aligned}
$$

which finishes the proof of (113).

Proof of Theorem 2.3. Let $0<\gamma<\gamma^{\prime}<\alpha, \gamma \leq 1$. We pick $\varepsilon=\min \left\{\varepsilon_{0}, \frac{\gamma^{\prime}-\gamma}{\gamma(d+2)}\right\}$. Then for any $0<s-t \leq \tau$ and $x, y \in \mathbb{R}^{d}$ such that $|x-y| \leq(s-t)^{1 / \alpha}$ we have

$$
\int_{\mathbb{R}^{d}}\left|p_{t, s}^{z}(x-z)-p_{t, s}^{z}(y-z)\right| d z \leq c|x-y|^{\gamma}(s-t)^{-\gamma^{\prime} / \alpha}
$$

where $c=c\left(\gamma, \gamma^{\prime}, \kappa(\tau)\right)$. To prove (114) we observe that our choice of $\varepsilon \in\left(0, \varepsilon_{0}\right]$ yields $1+(d+2) \varepsilon \leq \gamma^{\prime} / \gamma$. Hence, by (113), we get

$$
\left(\int_{\mathbb{R}^{d}}\left|p_{t, s}^{w}(x-w)-p_{t, s}^{w}(y-w)\right| d w\right)^{\gamma} \leq c|x-y|^{\gamma}(s-t)^{-\frac{\gamma}{\alpha}(1+(d+2) \varepsilon)} \leq c|x-y|^{\gamma}(s-t)^{-\gamma^{\prime} / \alpha} .
$$

On the other hand, by (81), we obtain

$$
\left(\int_{\mathbb{R}^{d}}\left|p_{t, s}^{w}(x-w)-p_{t, s}^{w}(y-w)\right| d w\right)^{1-\gamma} \leq c
$$

The last two estimates imply (114). 
If $|x-y| \geq((s-t) / 2)^{1 / \alpha}$ then the assertion of the theorem is trivial, so we may assume that $|x-y|<((s-t) / 2)^{1 / \alpha}$. We have

$$
\begin{aligned}
& \left|P_{t, s} f(x)-P_{t, s} f(y)\right|=\mid \int_{\mathbb{R}^{d}}\left(p_{t, s}^{z}(x-z)-p_{t, s}^{z}(y-z)\right) f(z) d z \\
& +\int_{t}^{s} \int_{\mathbb{R}^{d}}\left(p_{t, r}^{w}(x-w)-p_{t, r}^{w}(y-w)\right) \int_{\mathbb{R}^{d}} q_{r, s}(w, z) f(z) d z d w d r \mid .
\end{aligned}
$$

By (114) and (31), this is bounded from above by

$$
\begin{aligned}
& c|x-y|^{\gamma}(s-t)^{-\gamma^{\prime} / \alpha}\|f\|_{\infty} \\
& +c\|f\|_{\infty} \int_{t}^{s} \int_{\mathbb{R}^{d}}\left|p_{t, r}^{w}(x-w)-p_{t, r}^{w}(y-w)\right|(s-r)^{\varepsilon \alpha-1} d w d r .
\end{aligned}
$$

Recall that we assumed $|x-y|<((s-t) / 2)^{1 / \alpha}$. Let us denote

$$
\begin{aligned}
& \int_{t}^{s} \int_{\mathbb{R}^{d}}\left|p_{t, r}^{w}(x-w)-p_{t, r}^{w}(y-w)\right|(s-r)^{\varepsilon \alpha-1} d w d r \\
& =\int_{t}^{t+|x-y|^{\alpha}} \ldots+\int_{t+|x-y|^{\alpha}}^{t+(s-t) / 2} \ldots+\int_{t+(s-t) / 2}^{s-t} \ldots \\
& =\mathrm{I}+\mathrm{II}+\mathrm{III} .
\end{aligned}
$$

By (81) and our assumption $|x-y|^{\alpha}<(s-t) / 2$ we get

$$
\mathrm{I} \leq c \int_{t}^{t+|x-y|^{\alpha}}\left(\frac{s-t}{2}\right)^{\varepsilon \alpha-1} d r \leq c \frac{|x-y|^{\alpha}}{s-t} \leq c\left(\frac{|x-y|^{\alpha}}{s-t}\right)^{\gamma / \alpha} \leq c|x-y|^{\gamma}(s-t)^{-\gamma^{\prime} / \alpha} .
$$

By (114) we get

$$
\mathrm{II} \leq c|x-y|^{\gamma}(r-t)^{-\gamma^{\prime} / \alpha} d r \leq c|x-y|^{\gamma}(s-t)^{-\gamma^{\prime} / \alpha} .
$$

Again by (114) we obtain

$$
\mathrm{III} \leq c \int_{t+(s-t) / 2}^{s-t}|x-y|^{\gamma}(r-t)^{-\gamma^{\prime} / \alpha}(s-r)^{\varepsilon \alpha-1} d r \leq c|x-y|^{\gamma}(s-t)^{-\gamma^{\prime} / \alpha} .
$$

By (115) and the estimates of I, II, III we obtain the assertion of the theorem.

Lemma 5.18. For any $0<s-t \leq \tau$ and $x \in \mathbb{R}^{d}$ we have

$$
\sup _{y \in \mathbb{R}^{d}}\left|p_{t, s}^{(0)}(x, y)-\tilde{p}_{t, s}(x, y)\right| \leq c G_{s-t}(0)(s-t)^{\varepsilon}
$$

and

$$
\int_{\mathbb{R}^{d}}\left|p_{t, s}^{(0)}(x, y)-\tilde{p}_{t, s}(x, y)\right| d y \leq c(s-t)^{\varepsilon} .
$$

The contant $c=c(\kappa(\tau))$. If $\tau \leq \tau_{0}$, then $c=c(\bar{h})$.

Proof. Let $0<s-t \leq \tau, x \in \mathbb{R}^{d}$ be arbitrary. We have

$$
\begin{aligned}
\left|p_{t, s}^{(0)}(x, y)-\tilde{p}_{t, s}(x, y)\right| & =\left|p_{t, s}^{y}(y-x)-\tilde{p}_{t, s}(x, y)\right| \\
& \leq\left|p_{t, s}^{y}(y-x)-p_{t, s}^{x}(y-x)\right|+\left|p_{t, s}^{x}(y-x)-\tilde{p}_{t, s}(x, y)\right| \\
& =\mathrm{I}_{1}+\mathrm{I}_{2} .
\end{aligned}
$$


We also have

$$
\begin{aligned}
\mathrm{I}_{2} & \leq\left|\frac{1}{\left|\operatorname{det} A_{s}(x)\right|} G_{s-t}\left(\left(A_{s}(x)\right)^{-1}(y-x)\right)-\frac{1}{\left|\operatorname{det} A_{t}(x)\right|} G_{s-t}\left(\left(A_{s}(x)\right)^{-1}(y-x)\right)\right| \\
& +\frac{1}{\left|\operatorname{det} A_{t}(x)\right|}\left|G_{s-t}\left(\left(A_{s}(x)\right)^{-1}(y-x)\right)-G_{s-t}\left(\left(A_{t}(x)\right)^{-1}(y-x)\right)\right| \\
& +\frac{1}{\left|\operatorname{det} A_{t}(x)\right|}\left|G_{s-t}\left(\left(A_{t}(x)\right)^{-1}(y-x)\right)-\tilde{G}_{s-t}\left(\left(A_{t}(x)\right)^{-1}(y-x)\right)\right| \\
& =\mathrm{I}_{3}+\mathrm{I}_{4}+\mathrm{I}_{5} .
\end{aligned}
$$

It remains to justify $\sup _{y \in \mathbb{R}^{d}} \mathrm{I}_{i} \leq c G_{s-t}(0)(s-t)^{\varepsilon}$ and $\int_{\mathbb{R}^{d}} \mathrm{I}_{i} d y \leq c(s-t)^{\varepsilon}$ for $i \in$ $\{1,2,3,4,5\}$ and some $c>0$ for some $c=c(\kappa(\tau))$ in the general case or $c=c(\bar{h})$ if $t<s \leq \tau_{0}$.

By Lemma 5.8 we get such estimates for $\mathrm{I}_{1}$. By (8), (9) and (11) we get the estimates for $\mathrm{I}_{3}$. By Lemma 5.9 and (9) we obtain such estimates for $\mathrm{I}_{4}$. Analogous estimates of $\mathrm{I}_{5}$ follow from Lemma 4.8 and definitions of $G_{s-t}, \tilde{G}_{s-t}$.

\section{Appendix A. Estimates for Example 2.4 and Example 2.7}

In this section we prove the two inequalities, which were stated and used in Example 2.4 and Example 2.7.

Proof of (18). For $\rho_{k+1}<|y| \leq \rho_{k}$ we have $\rho_{k} \leq c^{-1}|y|$, hence

$$
\begin{aligned}
h(r) & =r^{-2} \sum_{k: \rho_{k} \leq r} \rho_{k}^{2} \nu\left(\rho_{k+1}<|y| \leq \rho_{k}\right)+\sum_{k: \rho_{k}>r} \nu\left(\rho_{k+1}<|y| \leq \rho_{k}\right) \\
& \leq c^{-2} r^{-2} \int\left(1 \wedge\left(|y|^{2} r^{-2}\right) \nu(d y)=c^{-2} \frac{4 c_{\alpha}}{\alpha(2-\alpha)} r^{-\alpha},\right.
\end{aligned}
$$

which proves the upper bound in (18). Similarly, we have

$$
h(r) \geq \int_{|y| \leq \rho_{1}}\left(1 \wedge\left(|y|^{2} r^{-2}\right) \nu(d y) \geq B r^{-\alpha}, \quad r \in(0,1],\right.
$$

which proves the lower bound.

Proof of (19). Without loss of generality we can take $t=0, x=0$, then

$$
X_{s}=\int_{0}^{s} A(r) \mathbf{e}_{1} d Z_{r}^{1}+\int_{0}^{s} A(r) \mathbf{e}_{2} d Z_{r}^{2}
$$

The characteristic function of $X_{t}$ has the form

$$
\phi_{s}^{X}(z)=\exp \left\{-\int_{0}^{s}\left(\left|(A(r) z)_{1}\right|^{\alpha_{1}}+\left|(A(r) z)_{2}\right|^{\alpha_{2}}\right) d r\right\},
$$

and thus the distribution density equals

$$
p_{s}^{X}(x)=\frac{1}{(2 \pi)^{2}} \int_{\mathbb{R}^{2}} \exp \left\{-i x \cdot z-\int_{0}^{s}\left(\left|(A(r) z)_{1}\right|^{\alpha_{1}}+\left|(A(r) z)_{2}\right|^{\alpha_{2}}\right) d r\right\} d z .
$$

In particular,

$$
p_{s}^{X}(0)=\frac{1}{(2 \pi)^{2}} \int_{\mathbb{R}^{2}} \exp \left\{-\int_{0}^{s}\left(\left|(A(r) z)_{1}\right|^{\alpha_{1}}+\left|(A(r) z)_{2}\right|^{\alpha_{2}}\right) d r\right\} d z,
$$

below we will show that the latter integral exists.

Lets estimate from below

We recall that

$$
\int_{0}^{s}\left(\left|(A(r) z)_{1}\right|^{\alpha_{1}}+\left|(A(r) z)_{2}\right|^{\alpha_{2}}\right) d r \geq \int_{0}^{s}\left|(A(r) z)_{2}\right|^{\alpha_{2}} d r
$$

$$
(A(r) z)_{2}=r^{\gamma} z_{1}+z_{2}, \quad z=\left(z_{1}, z_{2}\right)
$$


and perform case study.

Case 1: $\left|z_{2}\right|>\frac{s^{\gamma}}{2}\left|z_{1}\right|$. Then

$$
\left|r^{\gamma} z_{1}+z_{2}\right| \geq \frac{1}{4}\left|z_{2}\right| \text { if } r \in\left[0, \frac{s}{4^{1 / \gamma}}\right] \text { and }\left|r^{\gamma} z_{1}+z_{2}\right| \geq 0 \text { othwerwize, }
$$

which gives

$$
\int_{0}^{s}\left|(A(r) z)_{2}\right|^{\alpha_{2}} d r \geq c s\left|z_{2}\right|^{\alpha_{2}} .
$$

Case 2: $\left|z_{2}\right| \leq \frac{s^{\gamma}}{2}\left|z_{1}\right|$. Consider two intervals $I(s)=\left[\frac{s}{2}, \frac{3 s}{4}\right), J(s)=\left[\frac{3 s}{4}, s\right]$. At least one of these intervals is free from the roots of the function $r \mapsto\left|r^{\gamma} z_{1}+z_{2}\right|$, and this function depends on $v=r^{\gamma}$ linearly with the slope $\pm\left|z_{1}\right|$. Since the values of this function in the endpoints are positive, this yields that, at least on the half of the interval,

$$
\left|r^{\gamma} z_{1}+z_{2}\right| \geq c s^{\gamma}\left|z_{1}\right|
$$

which gives

$$
\int_{0}^{s}\left|(A(r) z)_{2}\right|^{\alpha_{2}} d r \geq c s^{1+\alpha_{2} \gamma}\left|z_{1}\right|^{\alpha_{2}} .
$$

Now we can complete the estimate of $p^{Y}(0)$. We have

$$
\begin{aligned}
p_{s}^{X}(0) & \leq \frac{1}{(2 \pi)^{2}} \int_{\mathbb{R}^{2}} \exp \left\{-\int_{0}^{t}\left|(A(s) z)_{2}\right|^{\alpha_{2}} d s\right\} d z \\
& \leq \frac{1}{(2 \pi)^{2}} \int_{\left|z_{2}\right|>\frac{s}{2}\left|z_{1}\right|} \exp \left\{-c s\left|z_{2}\right|^{\alpha_{2}}\right\} d z \\
& +\frac{1}{(2 \pi)^{2}} \int_{\left|z_{2}\right| \leq \frac{s}{2}\left|z_{1}\right|} \exp \left\{-c s^{1+\alpha_{2} \gamma}\left|z_{1}\right|^{\alpha_{2}}\right\} d z=: I_{1}+I_{2} .
\end{aligned}
$$

Since

$$
\begin{gathered}
I_{1}=\frac{4}{(2 \pi)^{2} s^{\gamma}} \int_{\Re}\left|z_{2}\right| \exp \left\{-c s\left|z_{2}\right|^{\alpha_{2}}\right\} d z_{2}=\left.\right|_{s^{1 / \alpha_{2}} z_{2}=v}=C s^{-\gamma-2 / \alpha_{2}}, \\
I_{2}=\frac{s^{\gamma}}{(2 \pi)^{2}} \int_{\Re}\left|z_{1}\right| \exp \left\{-c s^{1+\alpha_{2} \gamma}\left|z_{1}\right|^{\alpha_{2}}\right\} d z_{1}=\left.\right|_{s^{\left(1+\alpha_{2} \gamma\right) / \alpha_{2}} z_{1}=v} \\
=C s^{\gamma} \cdot t^{-2\left(1+\alpha_{2} \gamma\right) / \alpha_{2}}=C s^{-\gamma-2 / \alpha_{2}},
\end{gathered}
$$

this completes the proof of (19).

\section{REFERENCES}

[1] K. Bogdan, T. Grzywny, M. Ryznar, Density and tails of unimodal convolution semigroups, J. Funct. Anal. 266 (2014) 3543-3571.

[2] K. Bogdan, V. Knopova, P. Sztonyk, Heat kernel of anisotropic nonlocal operators, Documenta Mathematicae 25 (2020) 1-54.

[3] Z.-Q. Chen, Z. Hao, X. Zhang, Hölder regularity and gradient estimates for SDEs driven by cylindrical $\alpha$-stable processes, Electron. J. Probab. 25 (2020), article no. 137, 1-23.

[4] Z.-Q. Chen, E. Hu, L. Xie, X. Zhang, Heat kernels for non-symmetric diffusions operators with jumps, J. Differ. Equ. 263 (2017) 6576-6634.

[5] Z.-Q. Chen, X. Zhang, Heat kernels and analyticity of non-symmetric jump diffusion semigroups, Probab. Theory Relat. Fields 16 (2016) 267-312.

[6] Z.-Q. Chen, X. Zhang, Heat kernels for time-dependent non-symmetric stable-like operators, J. Math. Anal. Appl. 465 (2018) 1-21.

[7] F. H. Clarke, On the inverse function theorem, Pacific Journal of Mathematics Vol. 64, No 1 (1976), 97-102.

[8] A. Debussche, N. Fournier, Existence of densities for stable-like driven SDE's with Hölder continuous coefficients, J. Funct. Anal. 264(8) (2013) 1757-1778.

[9] S.D. Eidelman, S.D. Ivasyshen, A.N. Kochubei, Analytic Methods in the Theory of Differential and Pseudo-Differential Equations of Parabolic Type, Birkhäuser, Basel 2004.

[10] S. N. Ethier, T. G. Kurtz, Markov Processes: Characterization and Convergence, Wiley, New York 1986. 
[11] W. Feller, Zur Theorie der stochastischen Prozesse. (Existenz- und Eindeutigkeitssätze), Mathematische Annalen 113 (1936) 113-160. Reprinted and translated in R.L. Schilling, Z. Vondraček, W. Wojczynski, William Feller. Selected Papers I, Springer, Cham (2015).

[12] M. Friesen, P. Jin, B. Rüdiger, Existence of densities for stochastic differential equations driven by Lévy processes with anisotropic jumps, arXiv:1810.07504

[13] M. Gevrey, Sur les équations aux dérivées partielles du type parabolique, Journal des Mathematiques Pures et Appliquées 9 (1913) 305-471 and 10 (1914) 105-148.

[14] T. Grzywny, On Harnack inequality and Hölder regularity for isotropic unimodal Lévy processes, Potential Anal. 41 (2014) 1-29.

[15] T. Grzywny, K. Szczypkowski, Heat kernels of non-symmetric Lévy-type operators, J. Diff. Equ. 267 (2019) 6004-6064.

[16] T. Grzywny, K. Szczypkowski, Lévy processes: Concentration function and heat kernel bounds, Bernoulli 26(4) (2020) 3191-3223.

[17] J. Hadamard, Sur la solution fondamentale des équations aux dérivées partielles du type parabolique, Comptes Rendus de l'Academie des Sciences, Paris 152 (1911) 1148-1149.

[18] P. Hajłasz, Change of variables formula under minimal assumptions, Colloquium Mathematicum 64(1) (1993), 93-101.

[19] N. Ikeda, S. Watanabe, Stochastic differential equations and diffusion processes, North-Holland, Amsterdam, 1981.

[20] V. Knopova, A. Kochubei, A. Kulik, Parametrix Methods for Equations with Fractional Laplacians, In: A.N. Kochubei, Y. Luchko (eds.), Handbook of Fractional Calculus with Applications, Vol.2. De Gruyter, Berlin 2019.

[21] V. Knopova, A. Kulik, Parametrix construction of the transition probability density of the solution to an SDE driven by $\alpha$-stable noise, Annales de l'Institut Henri Poincaré 54(1) (2018) 100-140.

[22] V. Knopova, A. Kulik, R. Schilling, Construction and heat kernel estimates of general stable-like Markov processes, arXiv:2005.08491

[23] V. Knopova, R. Schilling, Transition density estimates for a class of Lévy and Lévy-type processes, J. Theoret. Probab. 25(1) (2012) 144-170.

[24] A.N. Kochubei, Parabolic pseudodifferential equations, hypersingular integrals, and Markov processes, Mathematics of the USSR - Izvestiya 33 (1989) 233-259.

[25] V. Kolokoltsov, Symmetric stable laws and stable-like jump-diffusions, Proc. London Math. Soc. 80 (2000) 725-768.

[26] F. Kühn, Lévy-Type Processes: Moments, Construction and Heat Kernel Estimates, Springer, Lecture Notes in Mathematics 2187 (Lévy Matters VI), Berlin 2017.

[27] F. Kühn, Transition probabilities of Lévy-type processes: Parametrix construction, Math. Nachr. 292 (2019) 358-376.

[28] T. Kulczycki, M. Ryznar, Semigroup properties of solutions of SDEs driven by Lévy processes with independent coordinates, Stochastic Process. Appl. 130 (2020) 7185-7217.

[29] T. Kulczycki, M. Ryznar, Transition density estimates for diagonal systems of SDEs driven by cylindrical $\alpha$-stable process, ALEA Lat. Am. J. Probab. Math. Stat. 15 (2018) 1335-1375.

[30] T. Kulczycki, M. Ryznar, P. Sztonyk, Strong Feller property for SDEs driven by multiplicative cylindrical stable noise, Potential Anal. (2020), published online https://doi.org/10.1007/s11118-020-09850-8

[31] A. Kulik, Approximation in law of locally $\alpha$-stable Lévy-type processes by non-linear regressions, Electron. J. Probab. 24 (2019), paper no. 83, 45 pp.

[32] A. Kulik, On weak uniqueness and distributional properties of a solution to an SDE with $\alpha$-stable noise, Stochastic Process. Appl. 129 (2019) 473-506.

[33] E.E. Levi, Sulle equazioni lineari totalmente ellittiche alle derivate parziali, Rendiconti del Circolo Matematico di Palermo 24 (1907) 275-317.

[34] D. W. Stroock, S. R. S. Varadhan, Multidimensional Diffusion Processes, Springer, Berlin 1979.

[35] P. Sztonyk, Estimates of densities for Lévy processes with lower intensity of large jumps, Math. Nachr. 290(1) (2017) 120-141.

Faculty of Pure and Applied Mathematics, Wroceaw University of Science and Technology, Wyb. Wyspiańskiego 27, 50-370 Wroceaw, Poland.

Email address: tadeusz.kulczycki@pwr.edu.pl

Email address: oleksii.kulyk@pwr.edu.pl

Email address: michal.ryznar@pwr.edu.pl 